ENVIRONHENTAL MONITORING REPORT

ZOR CALENDAR YEAR $T 984$

By

Joseph R. Stencel

May 1985

\section{DISCLAIMER}

This report was prepared as an aecount of bork sponsored by an agency of the Uniled States Government. Neither the United States Government nor any agency thereof, nor any of their emplayecs, makes any warranty, express or implied, or assumes any legal liability or responsibility for the accuracy, completeness, or usefulness of any information, apparatus, pfod tiel, or proness disclosed, or represents that its use would not infringe privately owned rights. Reference herein to any specific commetcial product, process, or service by trade name, trademark, manufacturer, or otherwise does not necessarily coastitute or imply its endorsemetin byeommendation, or favoring by the Udited Stales Govern ment or any agency thercof. The views and opinions of authors expressed berein do not necessarily state or reflect those of the Uniled States Government or any agency thereor. 


\title{
ENVIRONMENTAL MONITORING REPORT
}

FOA CAIENDAR YEAR 1984

Joseph R. Stencel

Plasma Physics taboratory, Princeton Univeritty

Princeton, New Jersey 08544

\begin{abstract}
The results of the environatental monitoring program for cyg4 for the Princeton Plasma Physics taboratory (PPPL) are presented ind discussed. The Princeton Large Torug (PLT), Princeton Beta Experiment (PBX), and PPPL's largest tokamak, the Tokatiak Fusion Test Reactor (TFTR) had a complete year of run time. In addition, the s-1 spheromak was in operation and the RE Test Facility came on-line. The phased approach of TFTR environmental monitoring continued with the addition of neutron monitorg. During cyg4 there were no adverse effects to the environment resulting from any operational program at ppel, and the Laboratory was in compliance with all applicable Federal. State, and local environatal reguiations.
\end{abstract}


1.0 INTRODUCTION

1. i

General

This report gives the regults of the environgental monitoring program at the Princeton university plasma Physics Laboratory (PPPL) for Calendar year 1994 (CY84). The report is prepared to provide the U.S. Department of Energy (DOE) and the public with Information on the level of radioactive and ronradioactive pollutants, if any, added to the environment as a result of PPPI operations. The objective of the environiental monitoring report is to document that DoE facility envizonmental protection programs adequately protect the environgent and the publis health.

PPPL has engaged in fugion energy research since 1951 and currently has three large tokamak devices in operation; namely, the rrinceton large Torus (PIT), the Princeton Geta Experiment (PBX), and the Tokamak Fusion Test Reactor (TFTR). In addition, the s-1 spheromaik began operation in crs3. The goal of the vagnetic Fusion program is to develop and demonstrate the practical application of fusion.

The Spheromak is a combination mirror/tokanak concept. The S-1 (F1g. 1) achieved lts initial goal of obtaining hot (100 ev) plasmas with lifetfmes of 1 msec or more. With toroldal currents of over $300 \mathrm{kA}$, electron densities in the mid to upper $10^{13}$ range, and with some 


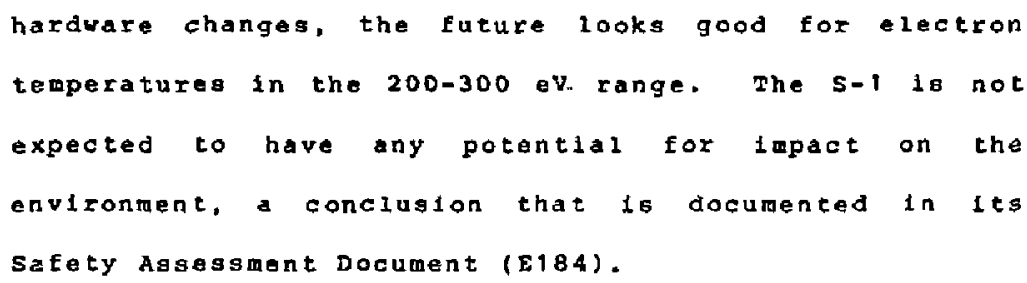

The pBX (Fig. 4) also reached one of its major milestones by achieving a s5z beta fratio of plasra pressure/magretic pressure). The combined progpt radiation to occupled areag frow PLT/gBx was less in Cr84 than in Cya3 because of different operating scenarios. A Safety Assessment Document for Pax published in 1984 Indicates that the PBX does not pose any potential environmental concerns (F184). 
The TFTR (Fig. 5), which had its firgt fuli yeat of running in CY83, Increased 1ts total neution production in cra4 to a level where radioactivity can be detected from activation of the vesgei after aajor run periods. The TETR is a torofdal device in which a tritium plasma will be magnetfcally confined at high vacuum and heated to extremely high temperatures by neutral beam injectors. The resulting $D-T$ fusion reactions will yield an output of energy equal to the energy required to heat the plasma. Because of recent fusion program budgetary constraints, the planned introduction of tritium into TFTR will be delayed from 1986 to 1988. The potential hazards of this prograd are addressed in required 5afety Analysis Reports (PSAR7B and FSARB2).

since the pppl site is, in general, open to the public. this free access has necessitated a thorough evaluation of the on-site discharges as well as the potential for off-site releases of radioactive or nonradioactive effluents. Thus, an extensive monitoring program tallared to these needs has been instituted and has been expanding over recent years. The PPPL environaental radiological monitoring prograd generally follows the guidance given in DOE reports A Gulde for: Environmental Radlological Surveillance at U.S. Department of Energy Installations (CoB1) and Environmental Dose Assessment Methods for Normal Operations at Dos Nuclear Sites (PNI-4410) (S:B2). This includes adherence to the standards jiven in COE orders, in particular, DOE order 5480.1. Chapter XI (DOEB1), whIch pertalns to 


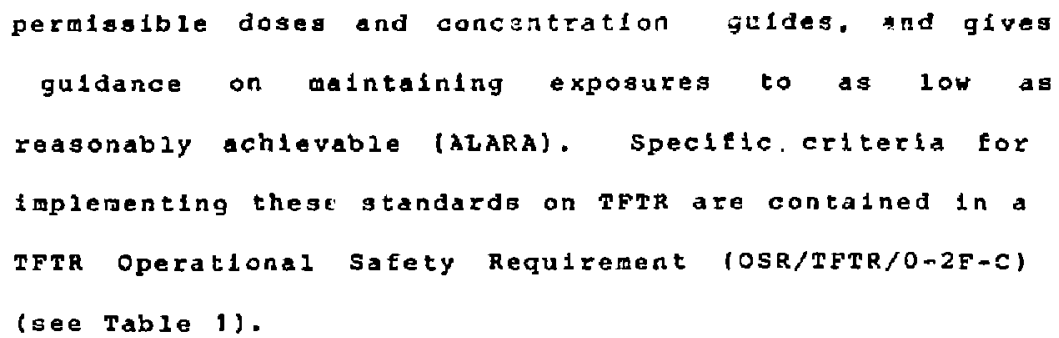


loproved methodologies were loplemented and a new rleteotological tower was installed and began operation In November 1383 (McB3). The firat year of windzose data from this new system is noted in this report ifigs. 6 and 7). The FSAR algo contains accident dose calculations for future operationg of TFTR with tritium. Tritium use, other than for system testing, wiz be delayed until 1988. The FSAR will be amended for tritium operation and will include changes since its original publication, Including any update with respect to new metecrologlcal and demographic data.

PPPL is located at the James Forrestal Reseacch camps of Princeton Untversity. As shown in Fig. B, the Location is in central NeH Jersey within Middiesex county. The ste has undisturbed areas with virgin forest, open grass areas, an airplane runway, and a smali brook running through its eastern boundary. The closest urban centers are New Brunswick, 14 miles to the northeast and Trenton, 12 miles to the southwest. Major metropolitan areag lncluding New York City, Philadelptia. and Newark are within 50 miles of the 5ite. As shown in Fig. 9, the municipalities of Princeton, Plainsboro, Kingston, Perns Neck, Princeton Junction, a.d Cranbury, among others, are in the 1mmedtate area of the site. ilso, the main campus of Princeton University is iocated primarliy within the borough of Princeton, approximately three miles to the 
west of the alte. The general layout of the facilities at the Forrestal campus is Indicated in Fig. 10 , including the D-site of the Plasma Physics laboratory, the apecific location of TFTR.

Figure 11 and Table 4 indicate the 1974 population estimates in sectors within ten miles of PQPL. Table 5 and Fig. 12 show the projected population for 1985 , which was derived by atculating the percentage increase projected for municipalities in the zero-to-ten-nile region by the Ney Jersey state offlce of Business Economics in the Department of Labor and Indugtry. The resldent population within 10 te 50 miles of pPpi, based on the 1970 sensus, $1 \mathrm{~s}$ shown in Table 6. The site is in the center of a highly urbanized region Erom Boston, Hassachusettg to Washington, D.C. The 1985 gopulation projections for the states of New Jersey, New York, and Penngylvania indicate a substantial population increase whin 50 miles of the PPPL site. The Princeton area is experiencing a substantial increase in new business moving into the Route 1 corridor near the site. Because of delays in the tritium program, a new demographic study wag alsa delayed until cyBt. 
2.0 SUMMARY

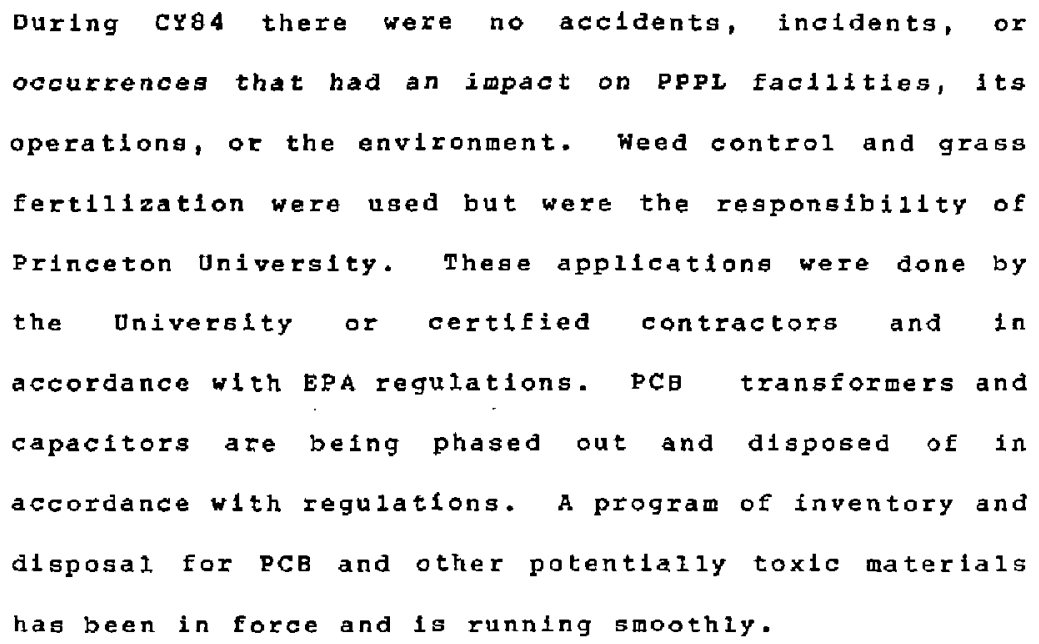

surface water analyses for both radioative and noniactoactive pollutants has not shown anything above normaly expected values. The 1nitial phase of a program for ground water analysis is slated to start in June 1985. Soll and vegetation samples were collected and analyzed to estabilsh baselinea. Radiation exposure ether via prompt or airborne radoastivity is still eagentialy nonexistent under present operations. Prompt radiation, however, is now detectable through the TFTR Bhield walis for the higher power pulses, and some activation of the vessel lo notes after a successful day of operations. Neutron monitors were added to the environmental ronitoring program in cys4, and othet monitors w111 be added this year as part of the phased approach to meet the requiremente of future operations. The radiological environmental program reviewed in cya4 
by the Idaho Nationa? Engtnoering Laboratory (INEL), at PPPL'g request, conflrmed that the program was adequateiy planned and meeting DOE requirements. Aithough future off-site expogure potential is expocted to re essentially nonexistent, a program to confirm this projection was recommended and lo presently being planned. COLLECTION FNALYSES, RND EVALUATION

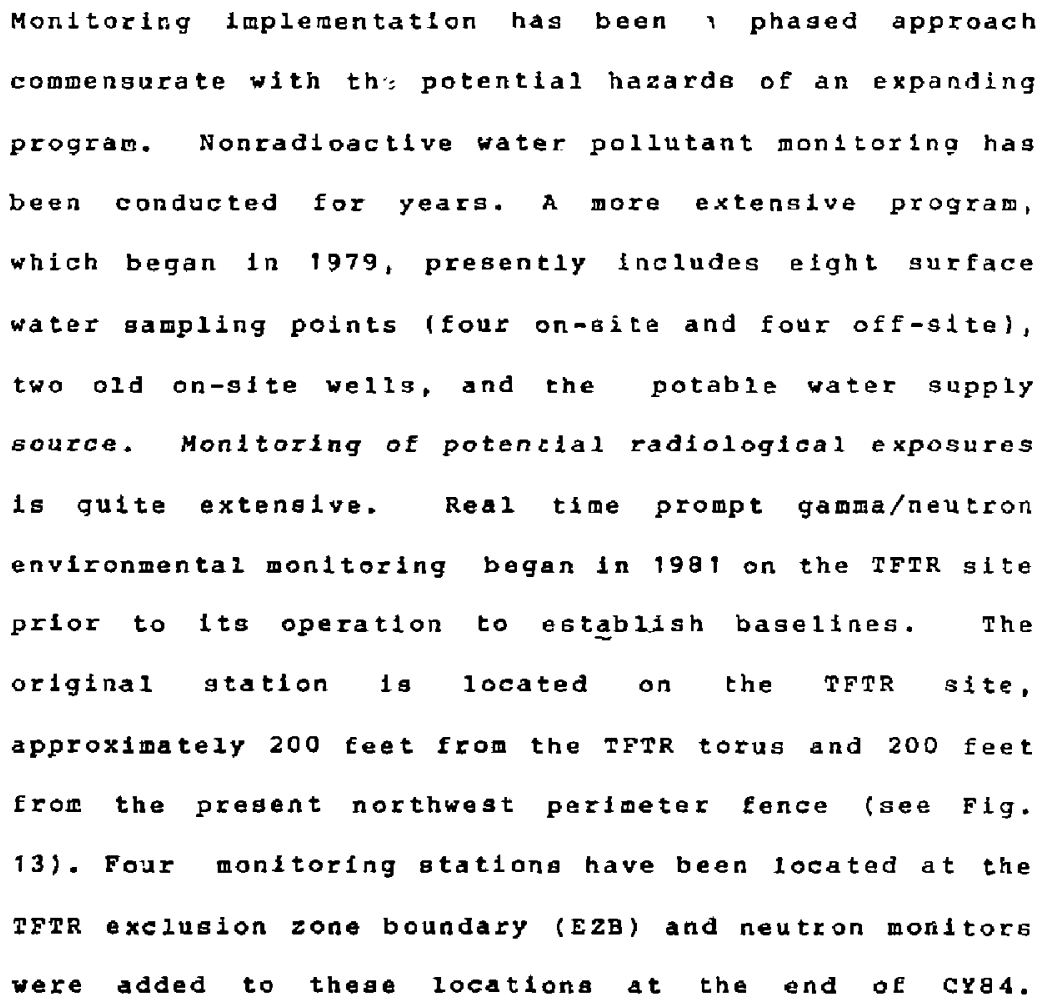


Radiological water bauples were taken at the oame locations a the nonradioactive gample polnts (see Figs. 13 and $(4)$.

\section{1 \\ RadioactIyIty}

\subsubsection{Penetrating Radiation}

Operation of the Princeton Large Torug (PLT) and the Pricaton geta sxperiment (PBX) cesults in the production of some penetrating radiation forimarliy bremstrahlung $x$ rays and neutronsl. Because neither the Pif or $P B X$ have a roof shield, skyshine radiation (primarily neutron) is seen ai the trit baseline sice Gon:toring station (RMS-B, Fig. 13). Shielding In place for the PLT/PBX has kept the total exposure dose equivalents in occupied areas below 200 mrem $(2$ mSv) in 1981 and 1982; and to 600 mrem (6msv) in 1983 durfng extersive PDX runs. The integrated value decreased to below 100 mrem (1mSv) in 1984 (5t85b). Actuai personnel exposure is much less than any of these values since io irdividual ocauples these areas during ali the pit or PBX runs.

The Laboratory has a policy that if occupational exposures have the potential to exceed 1000 mrem/yr 10 Hsv/y:l, the reapective project manager must petition the PDPL Executive Safety Board (ESB) for an exemptioa. In addition, the Laboratory appixes the DOE ELARA policy to a11 : s operations. 
In 1981 the measured exposure dose soove background at tha TFTR baseline monltoring station was 3.2 mrem 10.032 mSv). In 1982 the value was $3.3 \mathrm{mrom}(0.033 \mathrm{mSV})$. In t98.3 the value Increased to 6.9 mrem $(0.069 \mathrm{mSv})$, and in 1984 the value was 2 gred $(0.02 \mathrm{mSv})$ as the PLT/PBX operational scenailo changed.

The design objectfve of TFin is to Btay below 10 mrem/yr (0.1 w5y/yr) above neutraj background at 125 meterg from the machine from all sources of radiation. The Trta, like other tokamaks, produces bremsitrahlung $\ddot{x}$ radiation from the runaway electrang at the end of a puse. These $x$ rays, in the range of 0 to 20 Mev, also produce photoneutrons. Neutral beam injections with deaterium began at the end of Cr84. With these D-D runs the neutron fluxes will increase in CY85. While the total neu'ron production during CYB3 was on the order of $10^{15}$, tine dally rung in early crs5 are now reaching this value. Additional shielding will be added to the TrTa test cell waIls during cY8s. No significant penetrating radiation was added to the environs due to TFTR operations in 1984. Radlation levels (uR) were recorded for sove pulses ontside the test cell, with the total exposure dose equivalent at the TFTR slte boundary being less than 1 mrem $(0.01$ mSy) for CYa 4 (Sta5b). 
The TETR alte boundary moaltors are Reuter-stokes. Sentri 1011 pressuri:ed ionization chambers and He-3 moderated neutron detectors. The electronlcs in the lonization chambers are nodifled to allow the integration of any TFTR machine pulse prompt radiation that may be above natural background. These data are stoted and processed as part of the cICADA computer system. Four of these monitoring stations are placed at the E2B (125 meters from the TFTR) (see Fig. 13). Anothet eight ionization chambers of lower sensitivity are located neas the TFTR (four outside the test cell wall, three in the basegent, and one on the roofl. These eight detector locations are for personnel safety and are not considered environmental detectors pex se. Bes des the moderated He-3 neutron detectors, Bonner-type-moderated LI(I) detectors were used to montror at various locations throughout the TFTR facility. At the end of CY84 He-3 moderated newtron jetectors were placed at the same eight ionization chamber locastons around the test ce11. Figure 15 notes the net enviconmental gammand neutron measurements for CYBA. The 2 mrem for PLT, PBX, and TFTR operations were subtracted from these dato. This deta plot essentially confirts the ratural background levels expected in this part of New Jerspy. 
In Auguet 1980, EGEG, InC., under DOE contract, conducted an aerlas raglologlaal auruey of PPR and gurround ng axeas. The detection gystom uaed conglstod of 20 sodium lodide detectors, a multichannel analyzer, and a manetic tape recording syetem. The nowinal exposure rate range obnerved was elght to ten uR/hr. Detectod radiofatopes were consistent with normal backgraund emittera.

\subsubsection{Alrborne Radioattivity}

Radioactivation of aif and tritium in meagurabie concentrations lo not expected until pFra D-T operations. A real-time prototype baseline alt and paxticulate monitor has been in use gince the beginging of cyo2. This gystem consigts of two CaF(E⿰丿)木⿴囗十 phoswleh detectors which use gulse shape and tme varlations to give gross alpha, beta, and gamma information. A calloration has heen accompliahed for beta detection uging a xe-133 gas source. With the expertence gained wth this gyste四, it has bear decided that a bimple beta gelntiliator wil: provide adequato infortation. In crss ar and particulate monitars using beta detectorg w $\$ 1$ be procured for installation at the EZE. 
A differential tritium air monitor ueing an ethylene giyco butbler concept was found to be lradequate for environmental monitoring. A modified ollica gel syatog 1s under tast (Gr85) and will be deployed to provlde baseline data in concczt with the new trition schedules.

A review of our environmental radiation jrogram and the possible use of avaliable off-site monitoring by outside organizations was done by the Idaho National Engineering Laboratory (INEL) at PPPL's request in CYB4. Must of the off-site ionftoring stations of outside utilitis located in the directior of the prevailing winds were deemed to be too far away and therefore nat useful to our program. It ias augested tha several simple off-site trit+"lm =:-i alr particulate monftoring stations should be set up in lacal communities to provide a baseline data base to document our expected mintmal release during, operations with tritium. A silica-geltype monitor is undex teot for this option, and contacts are expected to be made rith the local commities riug cys5 on placement of these moniturs.

Meteorological data have been collected on-site at the original station since 1977. Lakehurst Naval Air staticn data were also acquired and were the primary data used in the mecoorological calculation ( $X / Q$ ) for the TFTR FSAR. DIBcusition on the problems of building wakes has led $\rightarrow$ a reevaluation of the meteorological date coltected on-site. In Nuvember 1983 a three-level, 60 meter tower was installed for gathesing 
meteorological data. The new windroge data from the first year of operation are shown in Figs. 6 and 7 . Preliminary analysis indicates that the alte is dominated by neutral to modecately stable conditions with woderately unstable to extregely unstable condtions oscurring less than a few percent of the time. Average aucface winds are ahout $2.1 \mathrm{~m} / \mathrm{a}$ and $\mathrm{Ise}$ to about $4.3 \mathrm{~m} / \mathrm{s}$ at $60 \mathrm{~m}, \mathrm{k} 085 \mathrm{~J}$. Baged on data from this new tower, $x / g$ values wil be recalculated for the tritium addendum to e FsAR before TFTR tritium operations.

\subsubsection{Waterborne Radioactivity}

\subsubsection{Surface Water}

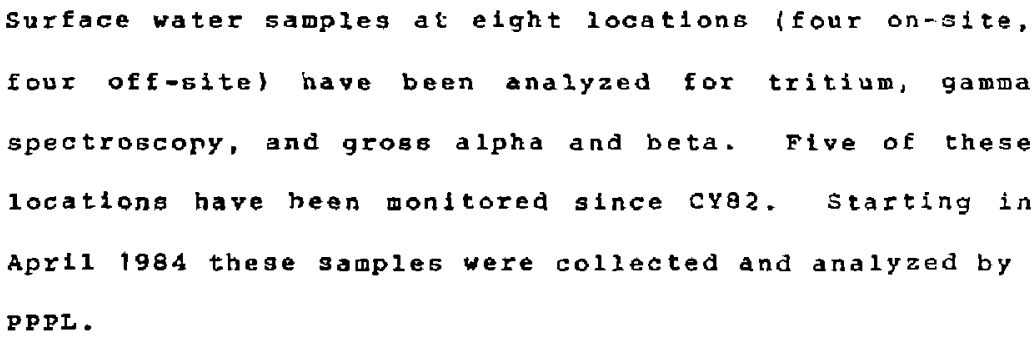

Dounstieam sampling occuts after mixing of effluent and ambient water is complete. Locations axe Indicated on FIgs. 13 and 14 . In January 1984 the surface water sampling increased from five to eight locationa as noted above and also included the potable water gupply. Typleal resulte aze ghown in Tabie 7 . 
sample analysis has shown no unusual background radioigotoper. Tritium analysis by liquid scirtiliation methods has shown tritium values to be less than 100 $\mathrm{pCi} / \mathrm{L}(13 \mathrm{kBq} / \mathrm{L})$ on all amples analyzed to date. Tritium enxichment procedures can be used to see lower levels and are presentiy under gtudy.

\section{1 .4 .2 Ground Hater}

Two existing on-slte wells (W4 and W5) were sampled In CYa4 (see Fig. 13). A more comprehenive ground water program will be initiated in June 1385 through the 0.5 . Geological survey. Thts program entails driling several monitoring wells on the TFTR gite in order to profile the ground water syoted for this site.

\section{1 .4 .3 Drinking Watex}

Potaile water ig supplied by the public utility, Elizabethtown water Co. In Afril 1984 a sample point at the input to PPPL was established (El location, Fig. 13). Radiactive analysis has included gama spectroscopy. tritiun, and gross alpha and beta.

\subsubsection{Fo욜tuff}

Because state regulatory requirements have essentially eximated small dairy farmers in this area, mills ls not a viable analysis medium around the site. Also the fish 
population ts very barce in Ditch 5 and Bee Brook, whleh carry the run-off from the site. A requegt to DoE was made in CYBA to eIfolnate thesg foodstuffa frow analya1s. A substitute of vegetables and honey that are avaliable from local ouroundingo ls uood. Inttial gamping Indicated tritiug levels below 100 $p(1 / 2(13 \mathrm{kgq} / \mathrm{L})$

\subsubsection{So11, Grass, Bottom Sediment}

Soll and grass samples collected $1 \mathrm{n} 1984$ indicated tritium levels below $100 \mathrm{pCl} / \mathrm{L}(13 \mathrm{kBq} / \mathrm{L})$. Laboratory techniques for doing these analysea were perfected in CY94 (Gr85). Surface soils and vegetations are one of the best indicators of teltium after a release (Jo74) $\{M \div 77),(M \pm 82\}$.

3.2 Nonradioactive Poliutants

\subsubsection{Alrborne Effluents (Stasa)}

PPQL has a New Jersey Department of Environmental Protection (NJDEP) Permit for its c-site bollers and f.el tank vents. The geven permit certificats numbers are 061294 through 061300 . In January 1985 a detailed review with NJDEP was undertaken. The result of these meetinga will be an amendment to the fuel tank vent permita In CY85 based on new data. Congiderations are a loo being given to require peritg for carpenter shop dugt collectors and Print shop exhaust. 
Meagurements of actual emision are not done; horever, emissions are calculated using formulae supplied by NJDER.

\section{2 .2 Water Ut1112ation (StB5a)}

\section{2 .2 .1 Potable Water}

Potable water is supplied by the publis utility, EI lzabethtown watet Co. PPPL (C-site) uses on the order of 55 million gallons per year. Water qualty analysis at the input to ppel was intiated in cyst for both rajioactive ind nonradioactive pollutants (Table 13).

\section{2 .2 .2 Process (nohpotable) Water}

Nongotable water is pumped by PRPL from the Delaware 8 Raritan Canal through a permit agreement with the New Jersey water supply Authority. This agreement provides PPPL the right to withdraw up to one million galions of water per day for process and fire flghting purposes for the perlod beginning July 1,1984 and ending on september 30, 1991. Treatment consists of filtration to remove suspended salids. Table 10 indlcates regults of water quality analygis at the canal. 


\subsubsection{Storw sawage}

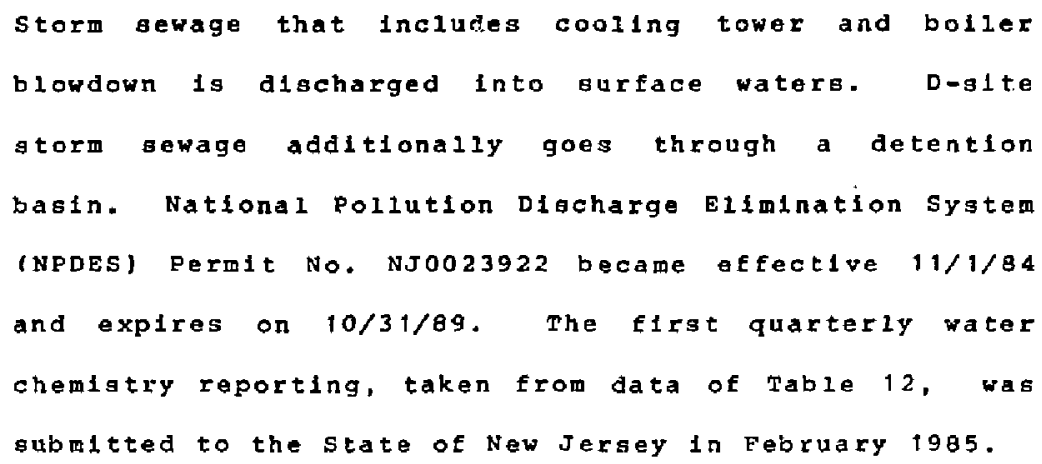




\section{$3,2,2,4$ Sanitary Sewage}

Sanitary awage is dicharged to the publicly owned treatment works operated by south Brunswick at stony Brook. Flow rates are measured by the pPR sanitary sewage metering station and average approximately 62,000 galiong per day. Sampling of ppid dicharges done by the publicly owned treatgent works has determined that pretreatment is unnecessary. Therefore, PPDL is in compliance with the EPA Pretreatment standara, $400 \mathrm{cr} 403$.

\subsubsection{Herbicides, Fertilizer, and Pesticides (C185)}

The use of herbicldes, pesticldes, and fertilizers is not controlled by Prol but by pzinceton university's grounds and bullding matntenance personnel. These are applied in accordance with state and Federal Regulations. Gerblcides are applied by a certified applicator. Table 19 litg the quantities applied durtng CY84.

\section{2 .4 Polychlorinated Biphenyis (PCB's)}

Beginning in CYB2 PPPi otarted a program to dispose of PCB capacitors, transfcimers, and contaminated items. All stored 1 tems in a GSA Warehouge in Belle Mead, New Jergey were dispoged of through approved contractors during the early phases of the program. Remaining PCB items were labeled as required and dn inventory, Inspection, ard status report program initiated. A 
Hazardous Materiel Coordinator was appointed to ensure

that any new ldentified leaking capacitors or trangformerg would be handled promptiy and properiy. At the beginning of CYB4 PPPL stili had 15 PCB trangformers and 6005 large capacitors. In cx84 375 large and 54 amall PCB capacitors were disposad of along with the oll and flushed carcass of two transformerg (HoBs). Funding 13 under conelderation for replacement of the other pce transformers. PCB capacitors are beling disposed of ae they are taken out of service. In late cras a pCB capacitor sprung a leak dustig a removal procedure. The PCBs were contained in the room of the splil and then cleaned up and disposed of through an approved waste contractor.

\subsubsection{Hazardous Wagtes}

Significant progress was made starting in cyBz with respect to Identification, collection, and disposal of hazardous waste in an environmentally acceptable manner. Haste disposal manifests are filed as required by the New Jersey Department of Environmental Protection, Division of Haste Management. Respongibility for this program rests Ith the PRP Hazardous Materiel Coordinator, under the Materiel Control Branch. A facility was aet up in CYB2 for temporary etorage of hasardoug materlalo. The area lnciudes an enclosed and 
covered area with a concrete containment, fire and security alarms, fire extingulghers, an eje wash, one emergency shower, and telephones. A new facility Is under consideration for cros.

\section{3 Environmental Impact}

\subsubsection{Asaegament of Radiation Doge to the Publ1c}

PPPL ig located In ane of the lesa densely populated areag in the metropolitan areas between New York City and Philadelphta. Frevious census data indicate that approximately 16 milion people live within 80 in is? miles) of the site, and approxtmately 225,000 within 16 km (10 miles) of pppt. This detalied distelbution of population as a Eunction of distance is given i: Tables 4-6. Because of ever lncreasing commercial growth in this area, a demographlc update was planned for cros. However, because of delays in TFTR trittum operations, it was felt that this update should be delayed in order to obtain the rust relevant data.

The overall exposure doae equivalent from all sources at the PPPL aite boundary was estimated to be less than 1 mrem for CY84 (st85b). Detalled person-rem calculations for the surrounding population were not performed, since the value would be insignificant in comparison to the approximately 100 mrem ( 1 mSv) each individual recelves from their natural background in New Jergey. 


\title{
3.3.2 Assesgment of Nonradioact1ve Pollutents
}

There were no activities during cyB4 that created problems with rempect to nonradioactivo pollutanta. A PCB capacitor spill and small oll optli were localized and cleaned up with no glgnificant environmental impact. The capacitor splil was contalned within a buliding and the oil spili had no effect on ground water.

Polychlorinated biphenylg (PCB's) continue to be disposed of $t n$ accordance with EPA requirenents. Herbicldes, pesticides, and fertilizers were used in very 11 mited quantities and are not expected to have any regative impact on the envisonment.

4.0 QUALITY ASSURANCE FOR LABORATORY ANALYSIS IN CY84

\begin{abstract}
Water samples for radioactivity, taken in the first quarter of cyas were analyzed by Teledyne Isotopes, 50 Van Buren Avenue, Wegtwood, New Jersey, During the last nine months of CYB4 the ample analygis was accomplished In-house. In general, In-houge procedures follow the HASL-300 Manual (VoB2). Teledyne Igotopes particlpated In the EPA (Lag Vegas) radioactive ga Program (KiB3). PRPI participateg in the SPA (Las Vegas) poogram as welI as a program provided by the Dog Environmertal Measurements Laboratory (EML) In New York City. These programe provide blind samples for analysts and subsequent camparion to known values and other
\end{abstract}


participants, analyis valuea. During cya4 PPPL Initiated a program to have les radiation counting laboratory certified by the state of New Jeraey through the EPA QA program.

rater analygis for nonradioactire constituents was done in-house for the first 11 months of cr84. Standard additions rere uged as a check on the quality of analyses. A program of using EPA QA sagples was being set up when the program was rotiched to an outside vendor, princecon lqua selence (PAS) of Edigon, NJ, In December 1984. PAS particlpates In a state of New Jersey QA program and has a quality assurance plan ( Ci\&2).

5.0 FUTURE PROGRAM EXPANSION

A new meteorological tower was installed in November 1983. Data Erom this sysiem will be used in updating dose calculations for the FSAR and will also be used in evaluating safety-related design requirements for any new D-T device at PPPL. Several dose assessment codes are being reviewed by PPPL with respect to using the data from thig tower. Thege codes lnclude AIRDOSE-ESA, an Ontario hydro version of TRITMOD, and PAVAN. Also a repiew of a draft report, pNL-4989, Interim Recommendations: Environmental Doge Calculationg for DoE Nuclear Sites, is waderway. 


\subsection{Mater quality}

The initial phases of a ground water monitoring program Is slated to begin in CY85. Water sample analyeis wil be added to both the racloactive and nonradioactive program ance wellg are drilied.

\subsection{Radioactive Effluento}

\subsubsection{Air Gaseous and Particulate}

A decision was made in cyas baged on data collected to limt the specific alr and particulate monttors to a beta deiector only. Several of these sygtems will be installed in cyo5. An environmental tritium monitor 1 a presently under test and will be reported on next year.

\section{3 .2 .2 Off-Site Radiological Monitoring}

off-site grab sajple water analysis is now an establighed pragram. A program for doing off-site tritium and particulate monitoring, primarily in the direction of the prevaliting wind direction, is befing planned.

\subsection{Nonradioactive}

Air effluents gtandards will continue to be bet by
following the quidelines of the state of Nev Jersey
Department of Environgental protection (NJDEP). Any


potential toxic materiato will be monttored and dispoged of in accordance with regulations and accepted gaiteifies. The detention basin update schedule for CY84 should become a real1ty in CYB5. An updated SPCC plan is in draft form and almogt ready for Implementation.

6.0 REgULATIONS AND SAFETY CRITERIA

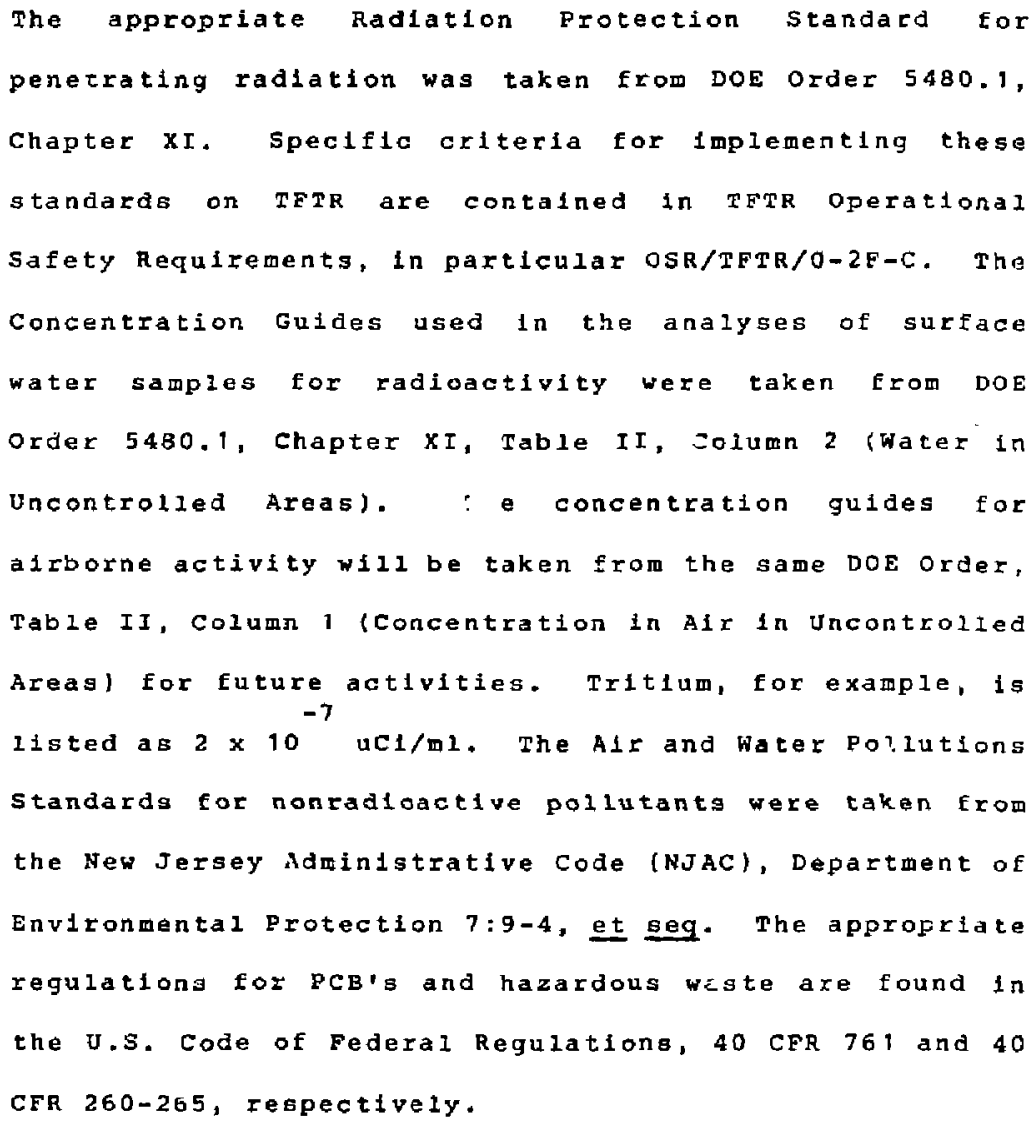


7.0 ACKNOHLEDMENTS

J. Ives (retired), G. Mitman, and J. Gilbert complied the data for radiation analysis; o. Griesbach performed in-houge radiation analygis; and $t$. Fennimoie worked on Instrument ingtaliation and calibration. These individuals are ataff members of the PPPL Project and Operational safety office, Health Physics section. K. Semel, the PPPL Indugtrial Hygleniet, rfulewed nonradioactive pollutant analysig dati. A. Silvestri did the nonradioactie water analysis through November 1984. N. Stecky is responsible for all water, storm, and sanitary sewage efforis. C. Pierce is involved in the specilication and procurement of radiation monttoring equipment. various other pepl individuala reviewad the manugcript.

This work supported by U.S. Department of Energy Contract No. DE-ACO2-76CHO3073. 


\section{REFERENCES}

Cib2 Cirelio, J., 1982, guality Agsurance Pian for the pAS Laboratory, Princeton Aqua science, New Brunswick, NJ.

CiB5 Clark, G.5., 1/25/65, "Chemicale," Princeton University Internal memo.

Co81 C rley, J.E., et al., 1982, A Guide for: Environmental Radiolczical. Survelitance at U.S. Department of Energy Instaliations, DOE/EP-023 iNational Pechnical Information Service).

DOEa1 DOE Order $5480.1, \mathrm{Ch} . \mathrm{XI}, \mathrm{B} / 13 / 81$, Environecital Protection, Safety and Heazth Protection Program EoI DoE operationg, D.S. Department of Energy, operational and Envifondental safety oivision.

E194 El21s, R., Jr. and Stencel, J.R., Eebruary 1984, Safety Assessment for the $S-1$ spheromak, Princeton Plasma Physica Iaboratory Report No. RPEI-20B3.

F184 Fleming, R.B., et aI. February 1984, Safety Assessment Document for the Princeton Beta Experiment, Princetan plasma Physics Labo:atjIy (unpublishedi.

FSAR82 Final Safety Analysis Report, Tokamak Fusion Test Reactor Facisities, Princeton Pl:s"a Physics Laboratory, i982.

Gr85 Griesbach, 0. , and Stenuei, J.R., January 1985, "An Environmetal Monttoring Program for a Eusion Test Reactor," Proceedings of the lath Midyear Topical Symposium of the Health Physics Society, Colorado springa, co.

HoB5 Holcombe, S., 1/1B/B5, "Annual Environmental Report," Princeton Plasma Physics Laboratory, internal wemo.

Jo74 Jordan, C.F., Stewar, M., and kIIne, J., 1974, "Tritiun Movement in Solis: The Impirtance of Exchange and Figh Initial Digpersion," Health Physicg, 27, 37-43.

K183 King, H.G., 2/17/83, "Quality Asgurance Intercomparison study," PPPI correspondence.

Ko85 Kolibal, J., April 1985, private communication, to be published at a PPPI report in 1985.

Me83 MeCarthy, E.F., anjErrera, L.J, B/15/B3, Final Monttoring Plan: Tokamak Fusion Test Reactor Meteorological Monitoring Program, TRC Environmental Consultants, Inc., 800 Connecticut Blvd., Eagt Haftford, CT 06108 .

Su77 Murphy, C.E., JI, Watts, J.R., and Corey, J.C., 1977 , "Environmenta 1 Tritium Transport Erom Atmospheric Release of Molecular Tritlum," Health Physlog, 33, 325-331. 
Mus2 Murphy, C.E., Ir., SHeet, C.H, and Falion, R.D., 1982. "Tritium Transport Around. Nuclear Facilities," Nuclear Safety. 23. $677-6.85$.

Na84 Nagy, A., and Beane, F., August 1984 , Safety Assegament for tha RF Test Facility, Princeton Plasma Physies Isporatory Report NO. PPP $\mathrm{I}-2129$.

PSAR78 Preliminary safety Analysib Report, erlnceton plasma Physics Laboratory Tokamak Fusion Test Reactor, 1978 .

StBl Stelner, P.A., 1991, An Aerial Radlologlcal survey of the Princetor Plasma Physics Laboratory and surrounding Area, U.S. DOE Remote Sensing Laboratory Report, EGG-1182-1806, UC-41.

St85a Stecky, N, 2/4/85, "Annual Enyironqental Report," PPPL internal memo.

Sta5b Stencel, J,R, 3/20/8S, "Eatimete of Radiation Exposure to occupied Areas for CY84 from PPPI Regearoh Activities," gPpL

internal report, JRS-291.

St.82 Strenge, D.L., Kennedy, H.E., Jr. and Corley, J.P., 1982, Environmental Dose Assessment Methods for Normal operations of DoE Nuclear Sites, PNL-4410/UC-11.

Vo82 Volctoin, 11,L, and de Piangue, G., 1962, EML Procedures. Manual HASI-30C, Depaetment of Energy, Environmental Heasurements Laboratory, 376 Hudson $5 t$, sY, NY 10014. 
TABLE 1

\section{TFTR RADIATION EXPOSURE CRITERIA}

The regearch facility radiation exposure criteria itemized In this table shall be Invoked for the TFTR only and 6 hall not be construed as being applicable to subsequent fugion test Eacilities, research faciltiles, or reactor projecta. The values quoted are for whole body exposures; however, the doE manual requirements for other organg (hands, arms, bones, etc.) and other time periods (quarterly) are also applicable to trir, and reductions between 1101 ts and degign objectives shall be consistent with those of the table.

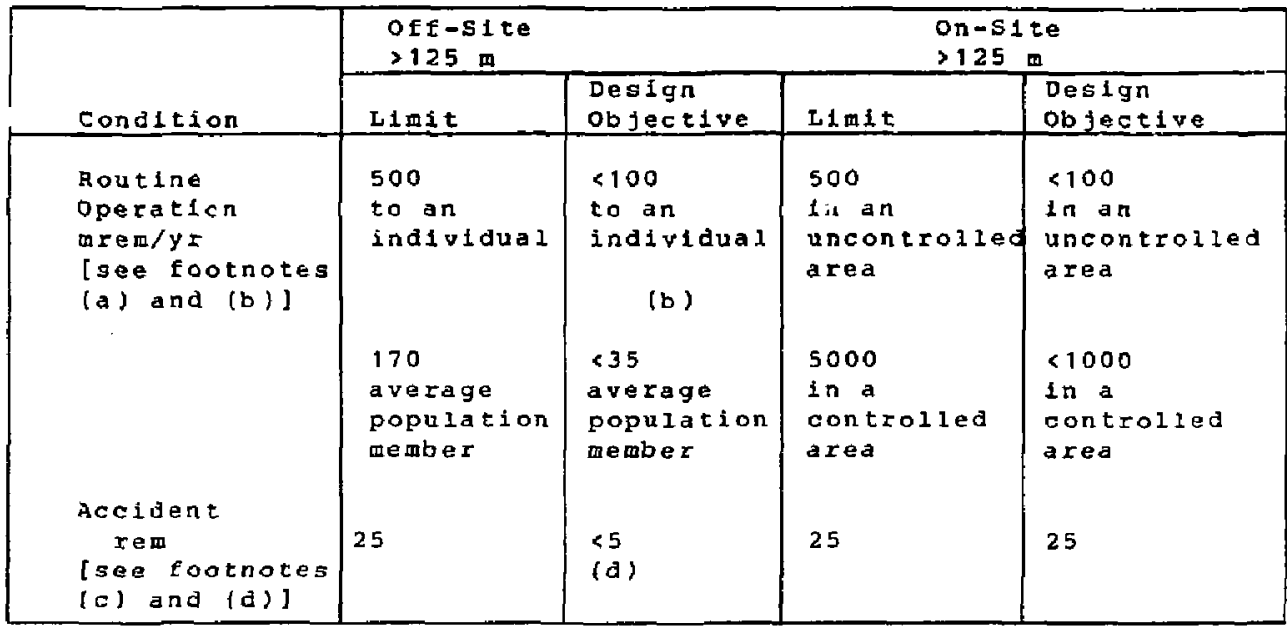

(a) Routine operation consists of:

(1) Normal camponent operation including shutdown, repair, mafntenance, checkout, adjustment, etc.

(2) Operational occurrences such as leaks, less of power, and

component malfunctions that are Iikely to occur once or more during the life of the facility.

(b) For normal operation, defined in (a) (1) above, the off-site design objective shall be $10 \mathrm{mrem} / \mathrm{year}$.

(c) Accident conditions conglat of:

(1) Very low probability events such as nmost intense predicte.t natural phenomena.

(2) Low probability events such as "nost probabie" natural phenomena and other component faliure events that are not likely to occur during the Iife of the facility.

(d) For those accident condifions defined by (c) (1) and (2) above, 1 rem. 
TABLE 2*

CRETICAL PATHWAYS

Path I.D.

A 1

A 2

A 3

L 1

L 2

L 3

L4 4
Digcharge Pathuay

Atmospheric $\rightarrow$ Whole Body Exposure

Atmospheric $\rightarrow$ Inhalation Exposiare

Atmospheric $\rightarrow$ Deposition on soll, Ingestion, whole Body Exposure

Ilquid

Water way $\rightarrow$ Drinking water

Supply $\longrightarrow$ Man

Liquid

Water Way $\rightarrow$ External Exposure

Liquid

Water Way $\rightarrow$ sediments $\rightarrow$

External Exposure

Iiquid

Water Way $\rightarrow$ Fish $\rightarrow$ Man 
TABI, 3 *

MONITORING PROGRAH COVERING CRITICAL PATHWAYS

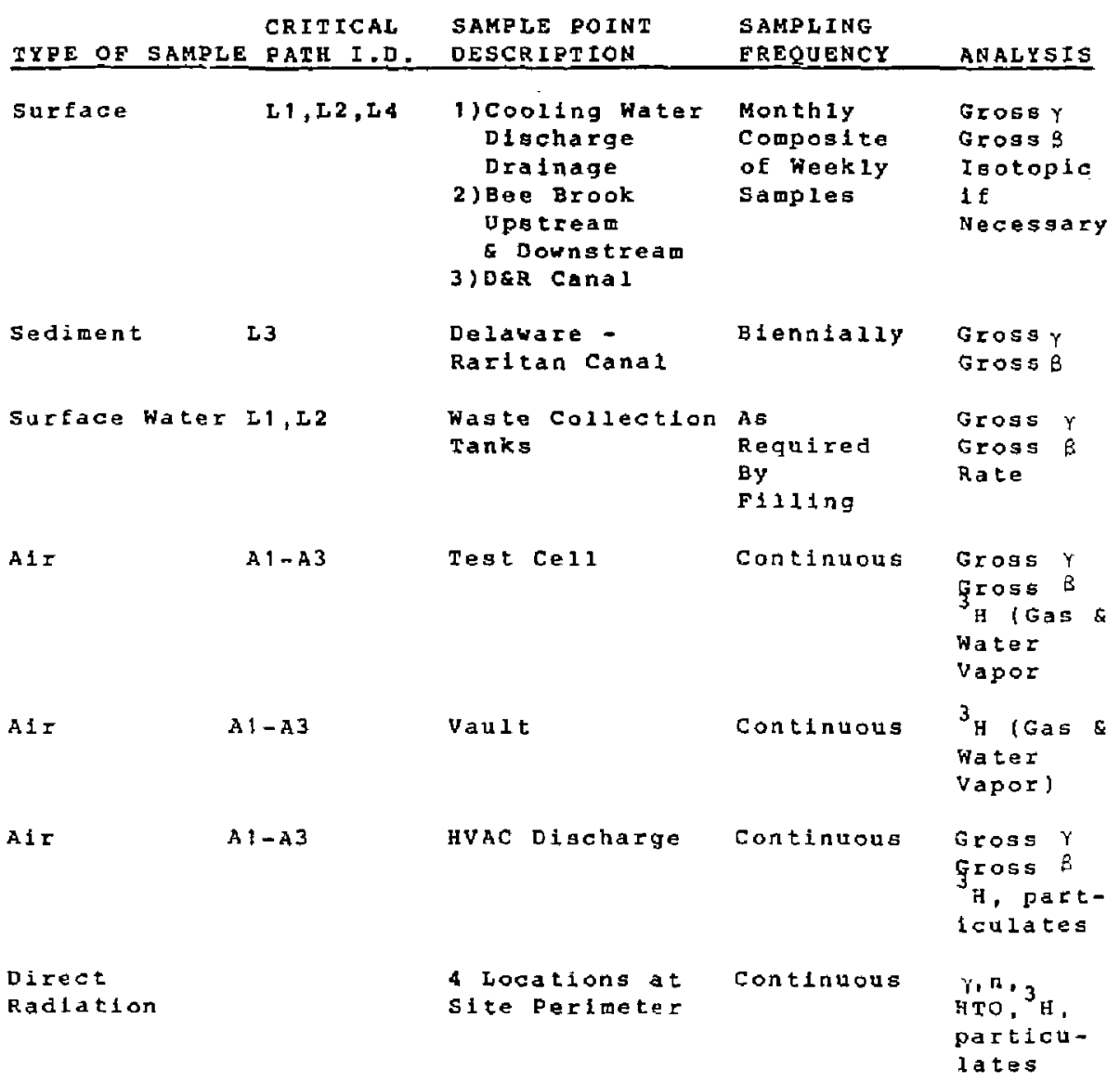

-TAKEN FROM TFTR FSAR TABLE 8.5-2 
TABLE 4:

1974 POPULATION ESTIMATES WTTHIM ANNOLAR SECTORS, $0-10$ MILES

TOTAL

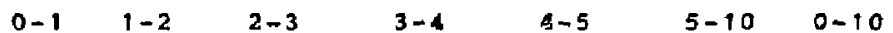

Sector Mile Mileg Miles Miles Miles Mileg Mileg sector

\begin{tabular}{|c|c|c|c|c|c|c|c|c|}
\hline $\mathbf{N}$ & 7 & 430 & 289 & 182 & 164 & 7.869 & 8,941 & $\mathbf{N}$ \\
\hline NNE & 0 & 22 & 144 & 137 & 3,284 & 10,353 & 13,940 & NNE \\
\hline NE & 7 & $5 B$ & 202 & 690 & 274 & $\uparrow 4,029$ & 15,260 & NE \\
\hline$E N E$ & 11 & 6 & 0 & 123 & 271 & 9,561 & 9,972 & ENE \\
\hline E & 0 & 0 & 55 & 25 & 43 & 11,796 & 11.919 & $\boldsymbol{E}$ \\
\hline ESE & 6 & 32 & 63 & 68 & 123 & 3,705 & 3,997 & ESE \\
\hline SE & 3 & 23 & 356 & 29 & 94 & 13,511 & 14,016 & SE \\
\hline$\$ \$ E$ & 6 & 2,859 & 49 & 27 & 71 & 13,837 & 15,849 & SSE \\
\hline 5 & 0 & 293 & 400 & 570 & 198 & 2,839 & 4,300 & $\mathbf{S}$ \\
\hline SSW & 0 & 95 & 688 & 1,221 & 530 & 26,112 & 28,646 & SSW \\
\hline Sw & 0 & 6 & 679 & 43 & 899 & 20,690 & 22,317 & SW \\
\hline WSW & 0 & 394 & 71 & 673 & 253 & 9,850 & 11,441 & WSW \\
\hline $\mathbf{H}$ & 3 & 835 & 5,712 & 5,832 & 695 & 7,586 & 20,663 & $W$ \\
\hline WNW & 0 & 437 & 2,889 & 1,455 & 294 & 6,788 & 11,863 & HNW \\
\hline$N W$ & 3 & 198 & 786 & 688 & 221 & $3,1 \mathrm{a3}$ & 5,079 & $N \mathbf{H}$ \\
\hline NNW & 3 & 415 & 259 & 144 & 698 & 2,354 & 4,373 & NNW \\
\hline TOTAL & 49 & 6,103 & 12,642 & 12,107 & 8,112 & 163,563 & 202,576 & TOTA \\
\hline
\end{tabular}

*TAKEN FROM TETR FSAR, TABLE 2.1.2-1 
TABLE 5*

1995 POQULATION PROJECTIONS WITHIN ANNULAR SECTORS, 0-10 MILES

$$
\begin{array}{llllll}
0-1 & 1-2 & 2-3 & 3-4 & 4-5 & 5-\frac{\text { TOTAL }}{10} \\
0-10
\end{array}
$$

Sector Mile Miles Miles Miles Miles Miles Miles sector

$\begin{array}{rrrrrrrrr}\text { N } & 9 & 529 & 355 & 229 & 205 & 9,850 & 11,177 & \text { N } \\ \text { NNE } & 0 & 27 & 177 & 169 & 4,041 & 13,008 & 17,422 & \text { NNE } \\ \text { NE } & 9 & 71 & 248 & 849 & 337 & 7,769 & 9,283 & \text { NE } \\ \text { ENE } & 16 & 8 & 0 & 151 & 333 & 12,406 & 12,914 & \text { ENE } \\ \text { E } & 1,217 & 0 & 74 & 31 & 53 & 13,956 & 15,331 & \text { E } \\ \text { ESE } & 9 & 46 & 1,600 & 78 & 144 & 4,157 & 6,034 & \text { ESE } \\ \text { SE } & 4 & 33 & 4,356 & 33 & 108 & 16,373 & 20,907 & \text { SE } \\ \text { SSE } & 9 & 4,117 & 71 & 35 & 82 & 16,018 & 20,332 & \text { SSE } \\ \text { S } & 0 & 548 & 456 & 650 & 226 & 3,788 & 5,669 & \text { S } \\ \text { SSW } & 0 & 137 & 784 & 1,392 & 604 & 30,893 & 33,810 & \text { SW } \\ \text { SW } & 0 & 9 & 774 & 49 & 1,025 & 24,987 & 26,844 & \text { SW } \\ \text { WSW } & 0 & 552 & 81 & 1,126 & 326 & 12,765 & 14,850 & \text { WSW } \\ \text { W } & 683 & 3,793 & 6,683 & 6,823 & 897 & 10,252 & 29,131 & \text { W } \\ \text { WNW } & 0 & 564 & 3,611 & 1,877 & 379 & 8,815 & 15,246 & \text { WNW } \\ \text { NW } & 4 & 255 & 1,014 & 860 & 263 & 3,815 & 6,211 & \text { NW } \\ \text { NNW } & 4 & 511 & 326 & 186 & 893 & 3,433 & 5,353 & \text { NNW } \\ \text { TOTAL } & 1,964 & 11,200 & 20,610 & 14,538 & 9,916 & 192,285 & 250,513 & \text { TOTAL }\end{array}$

-TAKEN FROM TFTR FSAR TABLE $2,1,2-3$ 
TABLE 6*

T970 RESIOENT POPULATIOH WITHIN ANNULAR SECTORS BETWEEN

\section{$10-50$ MILES}

\begin{tabular}{|c|c|c|c|c|c|c|}
\hline sector & $\begin{array}{r}10-20 \\
\text { Miles } \\
\end{array}$ & $\begin{array}{r}20-30 \\
\text { M11es } \\
\end{array}$ & $\begin{array}{l}30-40 \\
\text { M1 } 1 \text { eB }\end{array}$ & $\begin{array}{l}40-50 \\
M 110.5 \\
\end{array}$ & Tota I & sectox \\
\hline $\mathrm{N}$ & 69,330 & 35,961 & 143,546 & 60,073 & 308,910 & $N$ \\
\hline NNE & 133,838 & 237,191 & 354,551 & 544,621 & $1,270,201$ & NNE \\
\hline NE & 185,569 & 437.917 & $1,483,657$ & $3,893,770$ & $6,000,913$ & $N E$ \\
\hline ENE & 119,874 & 166,477 & $1,134,648$ & $1,629,062$ & $3,050,061$ & $E N E$ \\
\hline E & 33,077 & 112,389 & 69,706 & 0 & 215,172 & $\mathbf{E}$ \\
\hline ESE & 29,247 & 35,408 & 111,280 & 0 & 175,935 & ESE \\
\hline SE & 6,928 & 52,737 & 108,930 & 2,033 & 170,628 & SE \\
\hline S5E & 5.004 & 4,984 & 7,887 & 11,023 & 28,898 & SSE \\
\hline s & 5,933 & 61,238 & 7,349 & 3,935 & $7 B, 455$ & s \\
\hline SSW & 52,983 & 55,122 & 56,229 & $1 \pm 4,782$ & 279,116 & $s \leq t$ \\
\hline SW & 247,409 & 324,276 & $1,368,041$ & $1,220,152$ & $3,159,878$ & $\mathbf{S W}$ \\
\hline WSW & 35,985 & 117,952 & 310,930 & 289,105 & 753,972 & WSW \\
\hline W & 12,576 & 20,100 & 69,806 & 56,145 & 158,707 & W \\
\hline WNW & 6,054 & 10,112 & 15,399 & 254,563 & 286,128 & WNW \\
\hline NW & 12,819 & 12,698 & 66,455 & 85,836 & 177,808 & NW \\
\hline NNW & 14,429 & 14,462 & 34,875 & 14,937 & 78,703 & 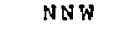 \\
\hline TOTAL & 971,055 & 104 & 39 & 0,037 & $16,193,485$ & $\mathbf{T}$ \\
\hline
\end{tabular}

* Taken from TFTR PSAR, Table $2.1 .2-4$ 
Table 7

TELEDYNE ISLTOPES

AEPCAT CF ANALYSIS

HORK ORCER NUHBER

$3-0830$
CUSTOHER P.O. NUMBER

Y4124-10
RUN CATE $03 / 27 / 86$

OATE RECE I VEO OELIVERY DATE

PAGE 1

$02 / 27 / 84 \quad 03 / 69 / 84$

MR JOE ST ENCEL
PRINCETON UNIVERSITY

PLASHA PHYSICS LAB

JAMES FDRR ESTAL CAHP US

P 0 BCX 451-HEALTH AND SAFETY C854C PRILCETON HJ

HATER

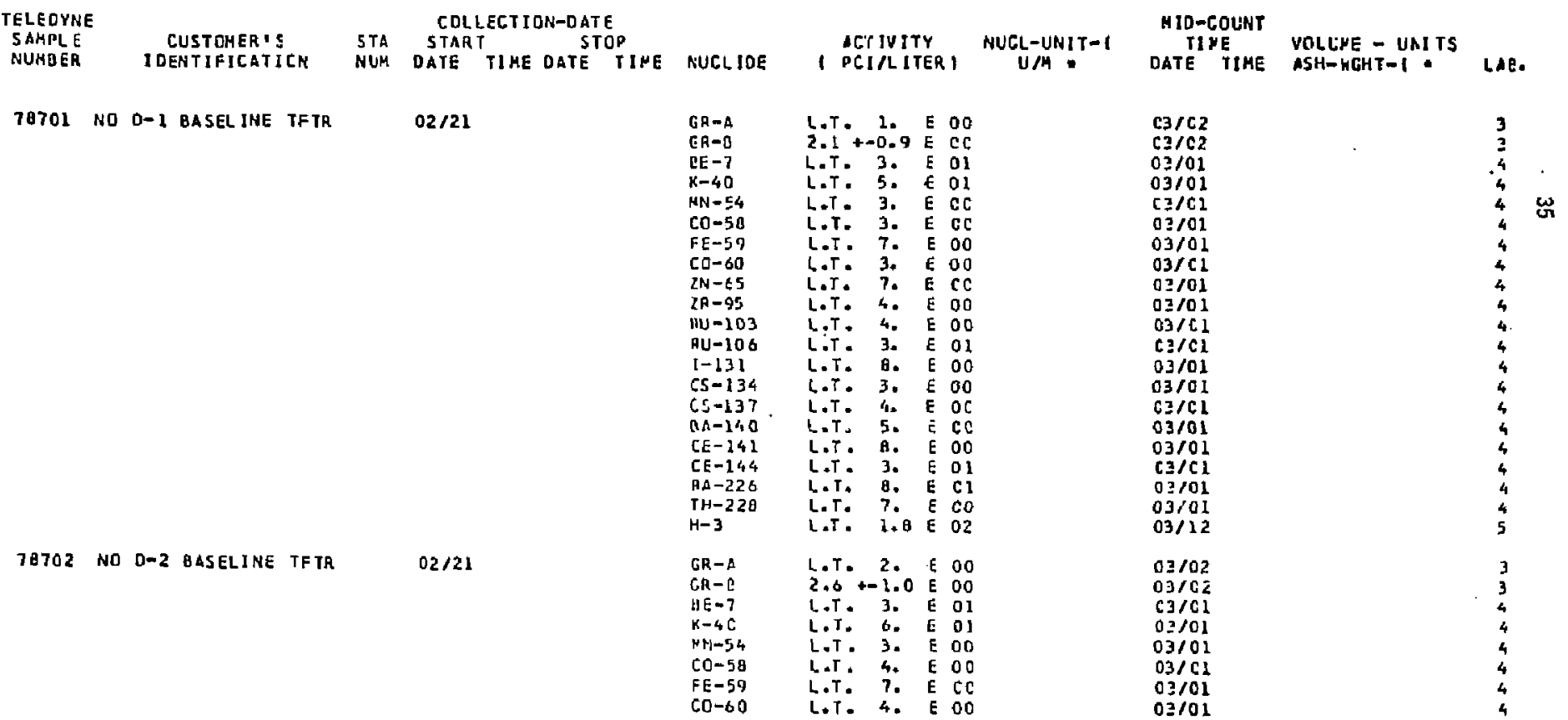


Table 7 (Cont'd)

TELEDYNE ISOTOPES

REPCA' OF ANALYSTS

WCRK OADEA NUMBER

$3-6830$
CUST DMER P.D. NUMBEA

Y4124-10
DATE RECEIVEO

$02 / 27,54$
RUN DATE 03/27/84

PAEE 2

HA JDE STENCEL

PRINCETON UNI VERSITY

PLASHA PHYSICS LAB

JAHES FORRESTAL CAHPUS

P O OOX 45I-HEALTH ANO SAFETY OESGC

PAINCETON HJ

H A T E R

\begin{tabular}{|c|c|c|c|c|c|c|c|c|c|c|c|c|}
\hline $\begin{array}{l}\text { TELEOYN } \\
\text { SAMPLE } \\
\text { NUHBER }\end{array}$ & & 105 & $\begin{array}{l}\text { CUSTOHER'S } \\
\text { ENTIFICATI }\end{array}$ & & $\begin{array}{l}\text { STA } \\
\text { AUH }\end{array}$ & $\begin{array}{l}\text { CQLLECTIO } \\
\text { START } \\
\text { OATE TINE }\end{array}$ & $\begin{array}{l}\text { ON OATE } \\
\text { STCP } \\
\text { DATE TIME }\end{array}$ & NUCLICE & ( $\stackrel{A C T}{P C T}$ & $\begin{array}{l}\text { IVIT } \\
\text { ILIT }\end{array}$ & ERs & $\begin{array}{c}\text { NUCL-UNIT-I } \\
\text { U/H }\end{array}$ \\
\hline 70702 & NO & $0-2$ & BASEL INE & TFTR & & $C 2 / 21$ & & $\begin{array}{l}2 N-65 \\
2 H-95 \\
R U-103 \\
A U-106 \\
1-131 \\
C S-134 \\
C S-137 \\
2 A-14 O \\
C E-14 L \\
C E-144 \\
A A-226 \\
T H-228 \\
H-3\end{array}$ & $\begin{array}{l}\text { L.T. } \\
\text { L.T. } \\
\text { L.T: } \\
\text { L.T: } \\
\text { L.T: } \\
\text { L.T: } \\
\text { L.T: } \\
\text { L.T: } \\
\text { L.T: } \\
\text { L.T: } \\
\text { L.T: } \\
\text { L.T: } \\
\text { L.T. }\end{array}$ & $\begin{array}{l}\text { 7. } \\
4 . \\
4 . \\
3 . \\
0 . \\
4 . \\
4 . \\
\text { a. } \\
0 . \\
\therefore: \\
\text { a. } \\
\text { i. }\end{array}$ & $\begin{array}{ll}E & 00 \\
i & 00 \\
E & 00 \\
E & 0 I \\
6 & 00 \\
E & 00 \\
E & C \\
E & C \\
E & 00 \\
E & 01 \\
E & C 1 \\
E & 0 \\
E & 02\end{array}$ & \\
\hline 28703 & NO & $B-1$ & GASELINE & IF TA & & $02 / 21$ & & $\begin{array}{l}G R-A \\
C R-B \\
C E-7 \\
X-40 \\
N N-54 \\
C Q-5 B \\
F E-59 \\
C Q-60 \\
I N-65 \\
2 R-95 \\
R U-103 \\
R U-106 \\
1-131 \\
C S-134 \\
C S-137 \\
C A-14 D \\
C E-142\end{array}$ & 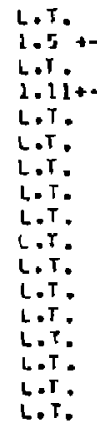 & $\begin{array}{l}1 . \\
0.8 \\
5 . \\
0.47 \\
6 . \\
6 . \\
1 . \\
6 . \\
1 . \\
6 . \\
6 . \\
5 . \\
1 . \\
7 . \\
6 . \\
1 . \\
1 .\end{array}$ & 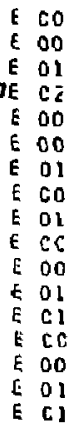 & \\
\hline
\end{tabular}

HIO-COLN

TIPE VOLUHE - UAITS DATE TIHE ASH-BCHT-I :

$02 / 01$

$03 / C 1$
$03 / C 1$

$03 / 61$

$03 / 01$

Ga/Cl

$C 2 / \mathrm{CI}$

03101

$03 / C 2$

C3/C1

$03 / 01$

$03 / 13$

$03 / 02$

$03 / 02$

C2/C1

62101

$03 / \mathrm{Cl}$

$\mathrm{C3} / \mathrm{Cl}$

03101

$03 / 01$

C3/C1

cict

03101

03101

c 3 ro.

031

$03 / 01$

$03 / C 1$

$C \geq / C 1$
$c \geq / 01$
248.

4 
Table 7 (Cont'd)

TELEOYAE ISOTOPES

REFCFT OF ANALYSIS

RUN DATE C3/2T/84

\begin{tabular}{|c|c|c|c|}
\hline HAR OPOER NUMAER & CUSTOHER P.O. NUMBER & DATE RECEIVEO & DELI YERY DATE \\
\hline $3-0830$ & $Y 4124-10$ & $02 / 27 / 64$ & $63 / 09 / 84$ \\
\hline
\end{tabular}

PAGE 3

MR JOE STENCEL

Y 4124-10

PLASHA PHYSICS LAB

JAMES FORAESTAL CAMPUS

$P$ O OOX 451-HEALTH AND SAFETY CB540

PAINCETON NJ

H A T E R

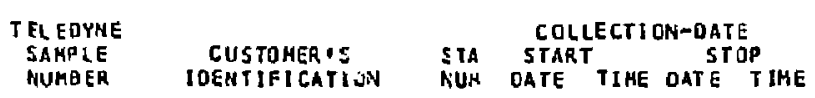

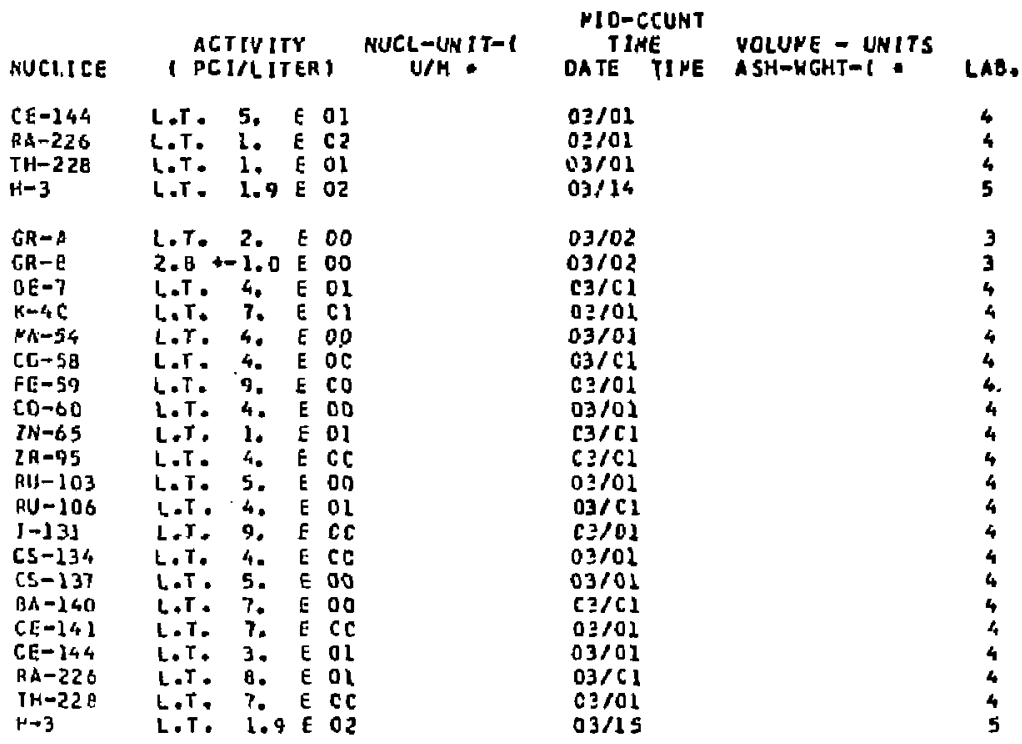


Table 7 (cont'd)

TELEOYNE ISOTCPES

REPCAT OF ANALYSIS

RLN DLTE C3/27/Q4

HCRK OROER NUHBER

CUSTOHER P.O. NUHBER

DATE RECEIVED

DELIVERY DATE

PACE *

MA JOE STENCEL

$3-\operatorname{Ca} 30$

$Y 4124=10$

$02 / 27 / 24$

$03 / 09 / 86$

PLASHA PHYSICS LAO

JAHES FORRESTAL CAHPUS

PO DOX 45I-HEALTH AHD SAFETY OESAC

PRINCETON NJ

\section{TEL EDYNE \\ SAMPLE}

CUSTOMER OS IOENTIFICATION

78705 NO C-1 BASELINE TFTR

76706 NO E-I BASEL INE TFTR

COLLECTION-DATE
STA START STOP
NUH DATE TIHE DATE TIME
C2/21

C2/21

$02 / 21$

\begin{tabular}{|c|c|c|c|}
\hline RU CLI IEE & $\begin{array}{l}\text { ACTJVIT } \\
\text { I PCILIT }\end{array}$ & $|x|$ & NUCL-UNIT-I \\
\hline $\begin{array}{l}C R-1 \\
G R-E \\
R E-7 \\
K=40 \\
K N-54 \\
C Q-5 B \\
F E-59 \\
C O-60 \\
2 N=65 \\
2 A-95 \\
P U-103 \\
R U-106 \\
1-131 \\
C S-134 \\
C S-137 \\
P A-140 \\
C E-141 \\
C E-144 \\
R A-226 \\
T H-228 \\
P=3\end{array}$ & 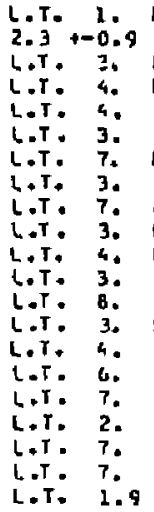 & 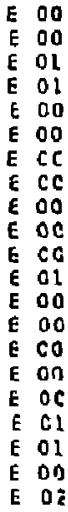 & 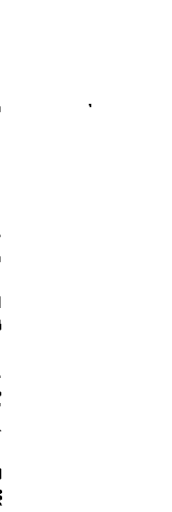 \\
\hline $\begin{array}{l}C A-A \\
C A-A \\
P F-7 \\
K=40 \\
N N=54 \\
C Q-5 B \\
F F=59 \\
C U-50 \\
C N=45\end{array}$ & 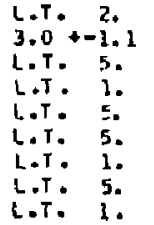 & 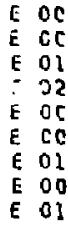 & \\
\hline
\end{tabular}

KIO-COLNT VOLUHE - UAITS DATE TIHE ASH-hGHT-I - LAE.

02102

$03 / \mathrm{Cz}$
$\mathrm{C} 3 / \mathrm{C2}$

02102

03102

CB/CE

carcz

$03 / 02$

$03 / 02$

c? 102

0.3102

$03 / 02$

$03 / \mathrm{C2}$

03102

03102

$c 3 / C 2$
$C 3 / C ?$

$03 / 02$

$03 / 02$

c 210 ?

02.16

$03 / C 1$

$02 / 01$

03101

03101

02101

$03 / \mathrm{Cl}$

C3/C1

C3/61
03 rol 
Table 7 (Cont'd)

TELEOYAE I SOTOPES

REPCET OF ANALYSIS

hCAK ofder numbea

$3-\cos 0$
CUSTOMER P.O. NUHEER
RUN DATE C3/27/BG

DATE RECEIVED OELIVERY DATE
$02 / 27 / 84$
c3/09/84

PR TNCETON UNIVERSITY
PLASMA PHYSICS LAB

PLASMA PHYSICS LAB
JAHES FORRESTAL CAHPUS

P O DOK 451-HEAL TH ANO SAFETY GE540

PR IHCETON NJ

H A T E A

TEL EDYNE

SAKPLE

CUSTOMER'S IOENTIFICATIOA

78706 NO E- 2 BASELINE TFTR

79707 NO M-J BASEL INE TFTR
CDLLECTION-DATE

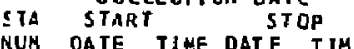
oAte tame date time nuClice $02 / 21$

$02 / 22$

\begin{tabular}{|c|c|c|}
\hline $\begin{array}{l}\text { PID-CCUNT } \\
\text { TIHE }\end{array}$ & YOLUME - UNITS & \\
\hline DATE TI HE & ASH-KGHT-I & LAB. \\
\hline $\begin{array}{l}63 / 01 \\
03 / 01 \\
03 / C 1 \\
03 / C 1 \\
03 / 01 \\
03 / 01 \\
63 / C 1 \\
63 / 01 \\
03 / 01 \\
03 / 01 \\
63 / C 1 \\
03 / 17\end{array}$ & & $\begin{array}{l}4 \\
4 \\
4 \\
4 \\
4 \\
4 \\
4 \\
4 \\
4 \\
4 \\
4 \\
5\end{array}$ \\
\hline $\begin{array}{l}03 / C 2 \\
C 3 / C 1 \\
03 / 02 \\
03 / C 2 \\
03 / 02 \\
03 / 02 \\
03 / 02 \\
03 / C 2 \\
03 / 02 \\
03 / 02 \\
63 / 02 \\
03 / C 2 \\
03 / 02 \\
03 / 02 \\
03 / C 2 \\
03 / 02 \\
03 / 02 \\
C 3 / C 2\end{array}$ & & $\begin{array}{l}3 \\
2 \\
4 \\
4 \\
4 \\
4 \\
4 \\
4 \\
4 \\
4 \\
4 \\
4 \\
4 \\
4 \\
4 \\
4 \\
4 \\
4\end{array}$ \\
\hline
\end{tabular}


Table 7 (Cont'd)

TELEOYAE ISOTDPES

REPCAT IF ANALYS IS

RUN CATE 03/27/84

LOAR ORDER NUHBER

$3-0.030$
CUSTOHER P.O. NUMAER

$Y 4124-10$
DATE RECEIUEO OELIVERY DATE

PAGE
YR JOE STEHCEL

BINCETON UMIVERSITY

$\because: A .5 H, 2$ HYSTCS LAO

-A.AES FORRESTAL CaMPUS

r. $30 X$ 45I-KEALTH ANO SAFETY $6854 C$

A - B. $E$ TON NJ

TELEDOHE

SAMPLE

NUMUER

CUSTOMEA'S

IOENTIFICATIEN

79707 NO M-I BASELINE TFTR

78TOE NO P-I BASELINE TFTR

78709 RE P-2 GASELINE IFTR
COLLECTION-OATE STA START TIME DATE TIME RUCbIOE $02 / 21$

$02 / 21$

02122

H I E R

$02 / 27 / 84 . \quad 53 / 09 / 84$

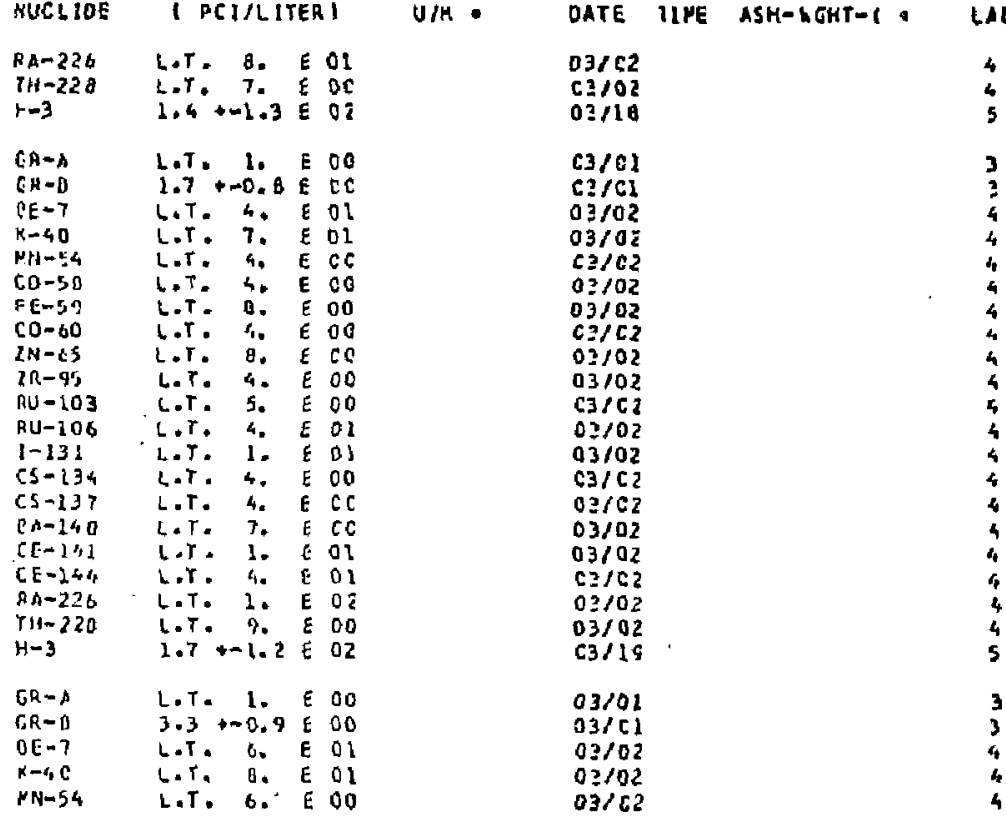


Table 7 (Cont'd)

TELEOYNE ISOTOPES

REPORT CF ANALYSIS

HORK OROER MUMBER

$3-\cos 30$
CUSTOMER P,O, NJMBER

$Y 4124-10$
AUN D/TE $03 / 27 / 84$

oAte reEE IVEO OEliveay oate

$03 / 05 / 84$

HA JOE STENCEL
PR INCETON UN IVER SITY

PRINCETON UN IVERSITY
PLASHA PHYSICS LAB

JAMES FORRESTAL CAMPLS

PD DOX 45I-HEALIH AND SAFETY 0E54C

PRINCETON NJ

H A T E R

TELEDYNE

SAHPLE

NUHLER

CUSTOMER'S I DENTIFICATIOH

COLLECTION-DATE

STA START

78709 NO P-2 BASELINE TFTR

TET10 NO H-4 GASELINE TFTR

$02 / 21$
$02 / 21$

\begin{tabular}{|c|c|c|c|c|}
\hline IDE & 1 & $\begin{array}{l}\text { IV } \\
\Lambda\end{array}$ & & \\
\hline $\begin{array}{l}C O-5 B \\
F E-59 \\
C Q-6 O \\
2 N=65 \\
2 R-9 S \\
A Y J-103 \\
F U-106 \\
I-131 \\
C S-134 \\
C S=137 \\
C A-14 O \\
C E=141 \\
C E-144 \\
R A=226 \\
T H=226 \\
H-3\end{array}$ & $\begin{array}{l}\text { L.T. } \\
\text { L.T. } \\
\text { L.T. } \\
\text { L.T. } \\
\text { L.T. } \\
\text { L.T. } \\
\text { L.T. } \\
\text { L.T. } \\
\text { L.T. } \\
\text { L.T. } \\
\text { L.T. } \\
\text { L.T } \\
\text { L.T. } \\
\text { L.T. } \\
\text { L.T } \\
2.4\end{array}$ & $\begin{array}{l}5 . \\
1 . \\
6 . \\
9 . \\
6 . \\
7 . \\
5: \\
2 . \\
7: \\
6 . \\
1 . \\
1 . \\
5 . \\
1 . \\
1 . \\
-1.3\end{array}$ & $\begin{array}{ll}E & 0 \\
E & 0 \\
E & 0 \\
E & 0 \\
E & 0 \\
E & 0 \\
E & 0 \\
E & 0 \\
E & 0 \\
E & 0 \\
E & 0 \\
E & 0 \\
E & 0 \\
E & 0 \\
E & 0 \\
E & \end{array}$ & $\begin{array}{l}C 0 \\
01 \\
00 \\
C 0 \\
00 \\
0 C \\
01 \\
01 \\
00 \\
C 0 \\
01 \\
01 \\
01 \\
02 \\
01 \\
02\end{array}$ \\
\hline $\begin{array}{l}G R-A \\
C A-E \\
B E-7 \\
X=4 C \\
Y N-54 \\
C O-5 D \\
F E-59 \\
C O-60 \\
Y N-65 \\
Z A-95 \\
A U=103 \\
R U-106 \\
I-131 \\
C S-134\end{array}$ & $\begin{array}{l}\text { L.T } \\
\text { L.T } \\
\text { L.T } \\
\text { L.T } \\
\text { L.T } \\
\text { L.T } \\
\text { L.T } \\
\text { L.T } \\
\text { L.T }\end{array}$ & $\begin{array}{l}-2.2 \\
-1.1 \\
4 . \\
5 . \\
4 . \\
4 . \\
9 . \\
4: \\
1 . \\
4 . \\
5 . \\
4 . \\
1 . \\
4 .\end{array}$ & $\begin{array}{l}\mathbf{E} \\
\mathbf{E} \\
\mathbf{E} \\
\mathbf{E} \\
\mathbf{E} \\
\mathbf{E} \\
\mathbf{E} \\
\mathbf{E} \\
\mathbf{E} \\
\mathbf{E} \\
E \\
\mathbf{E} \\
\mathbf{E} \\
\mathbf{E}\end{array}$ & $\begin{array}{l}00 \\
00 \\
02 \\
01 \\
00 \\
00 \\
00 \\
00 \\
01 \\
C C \\
00 \\
01 \\
C 1 \\
C 0\end{array}$ \\
\hline
\end{tabular}

MI O-COLNT UCL-UNIT-I $U / M$. DATE TIHE ASHARGHT-I? VOLLHE - UA!TS

\section{$03 / 02$}

$03 / 02$

c $3 / 62$

03702

$03 / \mathrm{C2}$

c3/02

c.? 3102

03702

$\mathrm{C} 3 / \mathrm{Cz}$

$03 / 62$

$03 / 02$

$03 / \mathrm{CZ}$

$\mathrm{C} 3 / 02$

$0 \geq 102$

$03 / 02$

$\mathrm{C} / 2 \mathrm{C}$

$03 / 01$

$03 / 01$

$6=102$
02102

व3द

$\mathrm{C3} / \mathrm{C} 2$

02102

$03 \% 02$

[3/C2

C $3 / \mathrm{CZ}$

$03 / 02$

$63 / 02$

03102

$03 / 02$ 
Table 7 (Cant'd)

TELEDYNE ISOTOPES

AEFCFT OF ANALYSIS

RUN OATE Q3/BT/84

MCRK DROER NUKBER

$$
3-5830
$$

HR JOE STENCEL

PA INCETON UNIVEASI TY

PLASHA PHYSICS LAD

JAHES FORRESTAL CAMPUS

$P$ O BOX 45I-HEALTH ANO SAFETY 0B5TOO

PRINCETON NJ

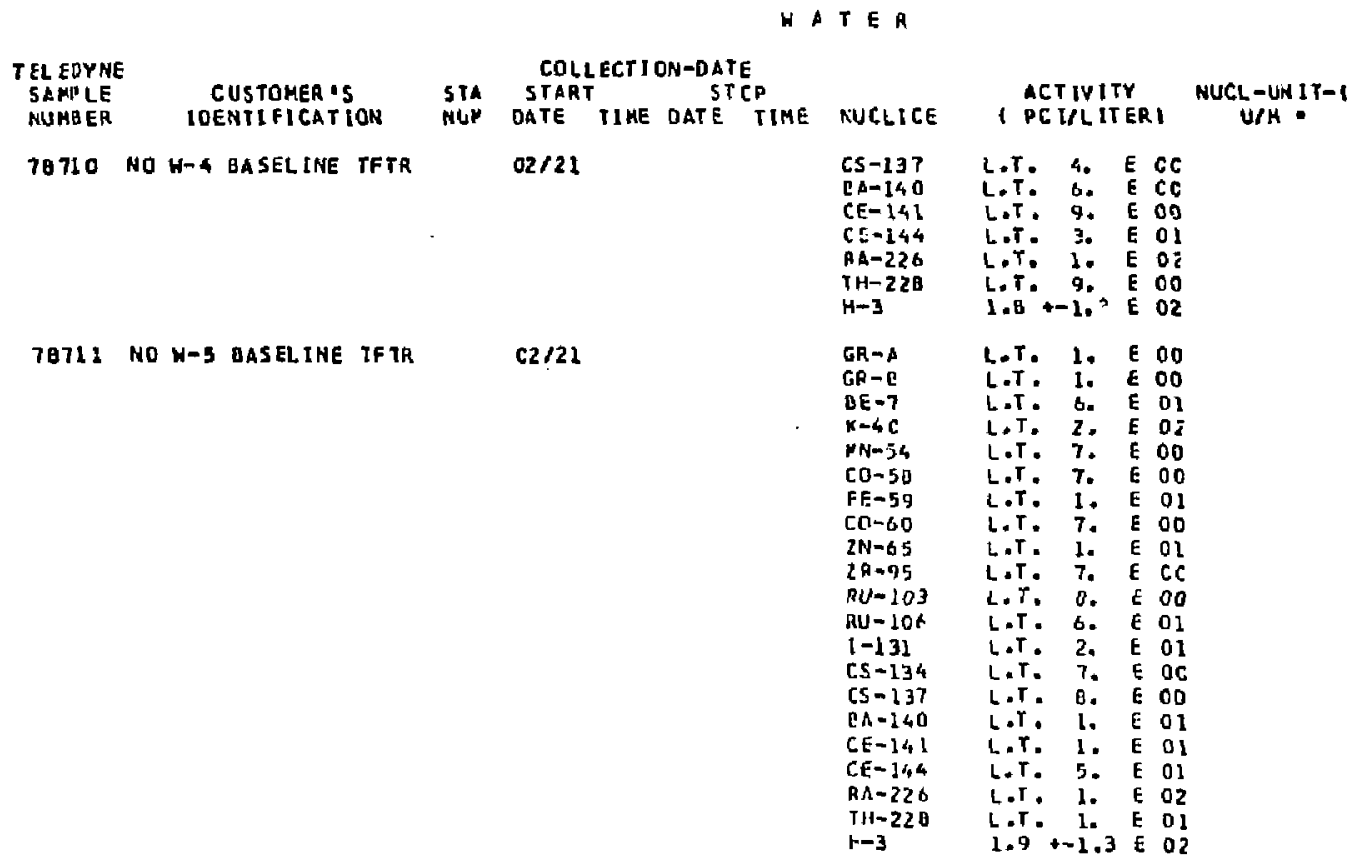

LAST PAEE OF REPORT

5 END 1 COPIES TO PRZ5TS MA JOE STENCEL

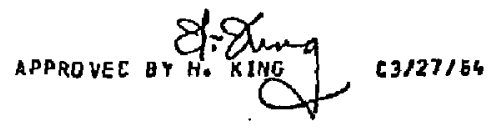

\begin{tabular}{|c|c|c|}
\hline $\begin{array}{l}\text { MIO-CCUNT } \\
\text { IIHE }\end{array}$ & YOLUAE = UHITS & (A) \\
\hline $\begin{array}{l}02 / 02 \\
03 / 02 \\
03 / C 2 \\
c 3 / 02 \\
02 / 02 \\
03 / 02 \\
63 / 21\end{array}$ & & $\begin{array}{l}4 \\
4 \\
4 \\
4 \\
4 \\
4 \\
5\end{array}$ \\
\hline 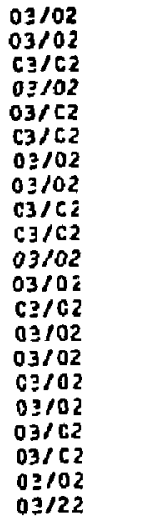 & & $\begin{array}{l}3 \\
3 \\
4 \\
4 \\
4 \\
4 \\
4 \\
4 \\
4 \\
4 \\
4 \\
4 \\
4 \\
4 \\
4 \\
4 \\
4 \\
4 \\
4 \\
5\end{array}$ \\
\hline
\end{tabular}

2 - gAS LAB.

3 - RAOIG Chemistay LAD.

4 - gellil gamma spec lab.

5 - TRITIUH GAS/L.S. LAB.

PAGE $B$ $03 / 09184$ 
Table 8

WATER QUALITY MONITORING DATA

forrestal Campus, Princeton University

Station: B1

Monitoring Station Location: Bee Brook, Location A

Parameter

Alkalinity, $\mathrm{mg} / 1 \mathrm{CaCO}_{3}$ eq.

$\mathrm{BOD}_{5}, \mathrm{mg} / 10$

Calcium Hardness, $\mathrm{mg} / 1 \mathrm{CaCO}_{3}$ eq. Chromium

Total, mg/1 Cr

Hexavalent, $\mathrm{mg} / \mathrm{lcr}$

$\mathrm{COD}, \mathrm{mg} / 1 \mathrm{O}$

Copper, $\mathrm{mg} / \mathrm{i} \mathrm{cu}$

Dissolved Oxygen, mg/ $\mathrm{O}_{2}$

EDrA, ing/a Cu eq.

Fecal Coliform Count, MPN/100 $\mathrm{ml}$ *

Flow, $\mathrm{ft}^{3} /$ sec

Iion, $\mathrm{mg} / 1 \mathrm{Ee}$

Nitrogen

Total Kjeldahl, mg/1 N

Ni trate, $m g / 1 \mathrm{~N}$

oil/Grease, Jng/1

ptI

Phosphate

Total, mg/1 P Or tho, mg/1

Solids

Dissolved, mg/ 1

Suspended, mg/

Sulfate, $\mathrm{mg} / 1 \mathrm{SO}_{4}$

Temperature, " $\mathrm{C}$

Iurbidity, NTU

zine, $\mathrm{mg} / \mathrm{i} \mathrm{zn}$
$1 / 10 / 84$

9:20 a.m.

44

6.2

38

0.05

0.01

6.6

0.021

13.6

6

3.3

0.30

0.46

0.84

1.6 .7

7.2

0.040

0.025

128
15
22
$5{ }^{\circ} \mathrm{C}$
7

0.035

\section{$1 / 24 / 84$}

$2 / 7 / 84$

10:00 a.m

38

1.5

36

FROZE

0.05

0.01

6.7

0.021

12.5

$-$

32

0.030

0.44

1.32

0.06

7.3

0.035

0.02

175

10

34

$0^{\circ} \mathrm{C}$

13

0.032
$3 / 6 / 84$

9:51 a.m.

38

2.4

28

0.05

0.01

10

0.021

10

0.095

16

1.2

0.34

0.64

1.25

0.98

7.1

0.018

0.012

116

6

16

$4^{\circ} \mathrm{C}$

14

0.026
$3 / 20 / 84$

10:08 a.m.

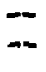

$-$

0.05

0.01

$-\infty$

$-$

$-$

2.2

$-$

$-$

7.0

0.06

0.01

116

7

24

$6^{\circ} \mathrm{C}$

8 
Table 8 (Cont'd)

WATER QUALITY MONITORING DATA

Eorrestal Campus, Princeton Univeralty

station: Bi

Monitoring Station Location: Bea Brook, Location A

\section{Parameter}

Alkalinity, $\mathrm{mg} / 1 \mathrm{CaCO}_{3} \mathrm{eq}$.

$\mathrm{BOD}_{5}, \mathrm{mg} / \mathrm{i} \mathrm{O}_{2}$

Calcium Hardness, mg/1 $\mathrm{CaCO}_{3} \mathrm{eq}$.

Chromium

$$
\text { Total, mg/1 cr }
$$

Hexavalent, mg/1 $\mathrm{Cr}$

COD, $\mathrm{mg} / \mathrm{l} \quad \mathrm{O}$

Copper, mg/l cu

Dissolved oxygen, mg/1 $\mathrm{O}_{2}$

EDTA, ng/ $1 \mathrm{Cu}$ eq.

pecal Coliform Count, MPN/100 ml.

Flow, $\mathrm{ft}^{3} / \mathrm{sec}$

Iron, $m y / 1$ Fe

Nì trogen

Total kjeldahl, mg/ $\mathrm{N}$

Nitrate, $\mathrm{mg} / \mathrm{I} \mathrm{N}$

oil/Grease, mg/1

pH

Phosphate

Total, mg/l $\mathrm{P}$

Ortho, ing/1

Solids

Dissolved, mg/1

Suspended, $\mathrm{mg} / 1$

Sulfate, $\mathrm{mg} / 1 \mathrm{so}_{4}$

Tenperature, ${ }^{\circ} \mathrm{C}$

Turbidity, NTU

zinc, $m g / 1$ an
4/03/84

11:14a.m.

18

3.2

22

0.05

0.01

13

0.01

10.5

56

1.7

0.52

0.66

0.38

0.95

7.0

0.66

0.022

126

8

18

$12^{\circ} \mathrm{C}$

0.044
$4 / 17 / 84$

10305 a.

--

$-$

0.05

0.01

$-$

$-$

$-$

70

1.1

0.24

$\rightarrow$

$-$

7.0

0.46

0.01

138

11

$1 \mathrm{~B}$

$13^{\circ} \mathrm{C}$

16 \begin{tabular}{l}
$5 / 8 / 94$ \\
$11: 25$ a. \\
\hline
\end{tabular}

\section{4}

1.2

28

0.05

0.01

18

0.01

11.2

$-$

114

0.9

0.42

0.32

0.83

0.42

7.0

0.04

0.01

124

15

22

$14^{\circ} \mathrm{C}$

18

0.036
$5 / 22 / 84$

10:30 a.m.

$-$

$-$

0.05

0.01

$-$

$-$

$-2$

128

0.44

$-$

$-$

7. 1

0.032

0.01

113

6

20

$10^{\circ} \mathrm{C}$

1.6

$-$
$6 / 5 / 84$

10:00 a.m.

32

1.6
26

0.05

0.01

32

0.010

9.2

-

170

1.75

0.74

0.42

0.94

0.44

7.0

0.06

0.04

116

a

18

$19^{\circ} \mathrm{C}$

5.7

0.025
$6 / 13 / 84$

10:35 a.m.

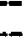

$-$

0.05

0.01

$-$

$-$

A

186

no flow

0.78

$-$

-

7.0

$0.0 \mathrm{~d}$

0.01

136

12

15

$21^{\circ} \circ$

1.6 
Table 8 (Cont'd)

WATER QUALIYY MONITORING OATA

Forrestal Campus, Princeton University

$$
\text { station: BI }
$$

Monitoring station Location: Bec Brook, Location A

\section{Parameter}

Alkalinity, mg/1 $\mathrm{CaCO}_{3} \mathrm{eq}$. $\mathrm{BOD}_{5}, \mathrm{mg} / 1 \mathrm{O}$

Calcium Hardness, mg/ $1 \mathrm{CaCO}_{3}$ eq. Chromium

\section{Total, $\mathrm{mg} / 1 \mathrm{Cr}$}

Ifexavalent, $\mathrm{mg} / \mathrm{i} \mathrm{Cr}$

coD, $\mathrm{mg} / 1$ o

Copper, $\mathrm{mg} / \mathrm{i} \mathrm{Cu}$

Dissolved Dxygen, $\mathrm{mg} / \mathrm{T} \mathrm{O}_{2}$

EDTA, mg/1 Cu eq.

Fecal Coliform Count, HPN/100 ml.

Flow, $\mathrm{ft}^{3} / \mathrm{sec}$

Iron; $\mathrm{mg} / \mathrm{l} \mathrm{Fe}$

Ni trogen

Total Kjeldahl, mg/1

Nitrate, $\mathrm{mg} / \mathrm{n}$

oil/Greage, mg/1

pH

Phosphate

Total, mg/ $\mathrm{P}$

ortho, mg/l $P$

Solids

Dissolved, mg/1

Suspended, mg/i

Sulfate, $\mathrm{mg} / 1 \mathrm{SO}_{4}$

Temperature, ${ }^{\circ} \mathrm{C}$

Turbidity, NiU

zine, Ing/1 $\mathrm{zh}$
$7 / 90 / 84$
$9: 15 a+m$

$7 / 24 / 84$

10:00 a.m.

9/7/84

10:40 a.m.

$8 / 28 / 84$

9:45 a.m.

$9 / 5 / 84$

10:00 a.m.

$9 / 18 / 84$

16

2.5

14

0.05

0.01

14

0.015

12

0.45

174

1.5

0.72

0.54

2.37

0.46

7.0

0.07

0.024

$+$

$\infty$

0.05

0.01

$-$

$-$

$-$

200

no flow

0.62

22
1.8

12

0.05

0.01

16

0.022

14

$0.4 C$

186

1.75

0.68

0.56

2.46

$-$

7.4

0.56

7.2

0.03

0.01

132

142
10

16

$20^{\circ} \mathrm{C}$

0.032
0.05

128

$+2$

18

$22^{\circ} \mathrm{C}$

2
$-$

\subsection{5}

0.01

--

$-$

$-$

136

1.75

0.42

18
1.2

12

0.05

0.01

12

0.02

14

0.44

B2

1.25

0.50

0.50

$-$

$\ldots$

7. 1

2.2

0.48

7.2

0.06

0.01

120

6

$19^{\circ} \mathrm{C}$

1.2

0.03
9:45 a.m.

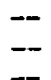

0.05

0.01

-.

$-$

78

NF

0.46

--

7.2

0.035

0.01

116

4

20

$19^{\circ} \mathrm{C}$

2.4 
Table 8 (Cont'd)

WATER QUALITY MONITORING DATA

Forrestal Campus, Princeton University

Station: BI

Mrnitoring station Location: Bee Bfook, Location A

\section{Parameter}

Alkalinity, $\mathrm{mg} / \mathrm{i} \mathrm{CaCO}_{3} \mathrm{eq}$.

$\mathrm{BOD}_{5}, \mathrm{mg} / \mathrm{l} \mathrm{O}_{2}$

Calcium Hardnegs, mg/1 $\mathrm{CaCO}_{3} \mathrm{eq}$.

Chromiura

Total, mg/ $1 \mathrm{Cr}$

Hexavalent, mg/1 Cr

COD, $\mathrm{mg} / 10$

Copper, $\mathrm{mg} / 1 \mathrm{Cu}$

Dissolved Oxygen, mg/l $\mathrm{O}_{2}$

EDTA, mg/1 Cu eq.

Fecal Coliform Count, MPN/100 ml.

Flow, $\mathrm{ft}^{3} / \mathrm{sec}$

Iron, $\mathrm{mg} / 1 \mathrm{Fe}$

Ni trogen

Total Kjeldahl, mg/1

Nitrate, mg/t $\mathrm{N}$

oil/Grease, mg/1

pH

Phosphate

$$
\text { Total, mg/1 } \mathrm{p}
$$

Solids

Jissolved, $\mathrm{mg} / 1$

Suspended, $\mathrm{mg} / 1$

Sulfate, $\mathrm{mg} / \mathrm{SO}$

Temperature, ${ }^{\circ} \mathrm{C}$

Purbiaty, NTU

Zine, mg/l $\mathrm{Zn}$

\section{$10 / 2 / 84$ \\ $10 / 16 / 84$ \\ $11 / 6 / 84$ \\ $11 / 20 / 84$ \\ 9: $30 \mathrm{a} \cdot \mathrm{Ant}$ \\ $12 / 31 / 84$ \\ 9:45 a.m.}

0.9

10

0.05

0.01

10

0.01

14

0.40

46

0.50

0.40

0.44

1.94

0.42

6.9

0.03

0.01

118

2

18

$15^{\circ} \mathrm{C}$

1.5

0.02

$-$

$-\cdots$

18

0.80

14

$-$

$-$

0.05

0.01

0.01

-

$-$

$-$

44

$\mathrm{NF}$

0.42

$-$

$-$

7.

7.4

0.04

0.01

124

3

20

$16^{\circ} \mathrm{C}$

0.8

0.02
0.05

0.01

12

0.02

14

0.40

48

1.25

0.48

0.48

1.7

$0.4 \mathrm{~A}$

7.3

0.04

0.0 .1

212

18

22

$15^{\circ} \mathrm{C}$

2.8

0.03
0.03

$<0.01$

14

0.02

$<0.08$

< 1

0.53

0.6

1.4

$<1$

7.0

0.03

0.02

83

3

22

5.5

0.02 
Table 9

WATER QUALITY MONITORING DATA

Forrestal Campus, Princeton Iniversity

Station: $\mathrm{B} 2$

Monitoring Staticn Location: Bee Brook, Location B

\begin{tabular}{|c|c|c|c|c|c|c|}
\hline & $1 / 10 / 84$ & $1 / 24 / 84$ & $2 / 7 / 84$ & $2 / 21 / 84$ & $3 / 6 / 84$ & $3 / 20 / 84$ \\
\hline Parameter & $9: 23 \mathrm{a} \cdot \mathrm{m}$. & 9:50 a.m: & 10:02 a.m. & 9:50 a. . . & $9: 56 \quad a . m$. & 10:10 a.m. \\
\hline Alkalinity, $\mathrm{mg} / 1 \mathrm{CaCO}_{3} \in \mathrm{q}$, & $3 \mathbf{9}$ & - & 46 & -- & 40 & - \\
\hline $\mathrm{BOD}_{5}, \mathrm{mg} / 1 \mathrm{O}_{2}$ & 3.8 & - & 9.8 & -- & 4.4 & - \\
\hline $\begin{array}{l}\text { Calcium Hardness, mg/1 } \mathrm{CaCO}_{3} \text { eq. } \\
\text { Chromium }\end{array}$ & 36 & -- & 34 & -- & 38 & - \\
\hline Total, $\mathrm{mg} / 1 \mathrm{Cr}$ & 0.05 & 0.05 & 0.05 & 0.05 & 0.05 & 0.05 \\
\hline Hexavalent, mg/1 $\mathrm{Cr}$ & 0.01 & 0.01 & 0.01 & 0.01 & 0.01 & 0.01 \\
\hline $\mathrm{COD}, \mathrm{mg} / 1 \mathrm{O}_{2}$ & 10.5 & - & 10.1 & -- & 16 & - \\
\hline Copper, mg/i $\mathrm{Cu}$ & 0.025 & - & 0.025 & - & 0.013 & - \\
\hline Dissolved Oxygen, $\mathrm{mg} / 1 \mathrm{O}_{2}$ & 12.5 & - & 12.6 & $-\infty$ & 14 & 22 \\
\hline EDPA, mg/1 Cu eq. & 0.088 & $\because$ & 0.035 & - & 0.035 & .4 \\
\hline Fecal Coliform Count, MPN $/ 100 \mathrm{ml}$. & 2 & 12 & 16 & 22 & 14 & 0.43 \\
\hline Flow, $\mathrm{ft}^{3} / \mathrm{sec}$ & 0.40 & 0.50 & 0.4 & 0.5 & 0.4 & - \\
\hline $\begin{array}{l}\text { Iron, mg/1 Fe } \\
\text { Nitrogen }\end{array}$ & 0.42 & 0.48 & 0.32 & 0.46 & 0.46 & - \\
\hline Total kjeldahl, mg/l $\mathrm{N}$ & 0.55 & - & 1.56 & -- & $1 \cdot 34$ & -- \\
\hline Nitrate, $m g / 1 \mathrm{~N}$ & 0.98 & - & 1.76 & -- & 0.85 & $\rightarrow$ \\
\hline oil/Grease, i mg/1 & 1.5 & - & 2.1 & -- & 9 & - \\
\hline $\mathrm{PH}$ & 7.1 & $7 \cdot 3$ & 7.2 & 7.3 & 7.0 & 7.0 \\
\hline Phosphate & & & & & & \\
\hline Total, mg/l $\mathrm{P}$ & 0.16 & 0.18 & 0.29 & 0.29 & 0.15 & 0.02 \\
\hline Ortho, $\mathrm{mg} / \mathrm{l} \mathrm{P}$ & 0.088 & 0.065 & 0.16 & 0.064 & 0.032 & 0.013 \\
\hline Solida & & & & & & \\
\hline Dissolved, mg/1 & 144 & 136 & 116 & 128 & 120 & 112 \\
\hline Suspended, mg/1 & 16 & 18 & 12 & 14 & 10 & 13 \\
\hline sulfate, $\mathrm{ng} / 1 \mathrm{So}_{4}$ & 29 & 14 & 25 & 22 & 24 & 27 \\
\hline Temperature, ${ }^{\circ} \mathrm{C}$ & $9^{\circ} \mathrm{C}$ & $\mathrm{H}^{\circ} \mathrm{C}$ & 62 & $8^{\circ} \mathrm{C}$ & $7^{\circ} \mathrm{C}$ & $y^{\circ} \mathrm{C}$ \\
\hline Turbidity, N'SU & $a$ & $a$ & 12 & 10 & 16 & 13 \\
\hline Zine, mg/1 $2 n$ & 0.034 & -- & 0.032 & - & 0.032 & -- \\
\hline
\end{tabular}


Table 9 (Cont'd)

HATER QUALITY MONITORING DATA

Forrestal Campus, Princeton University

Station: $\quad 82$

Monltoring station Location: Bee Brook, Location B

\section{Parameter}

Alkalinity, $\mathrm{mg} / 1 \mathrm{CaCO}_{3} \mathrm{eq}$. $\mathrm{BOD}_{5}, \mathrm{mg} / \mathrm{i} \mathrm{O}_{2}$

Calcium Hardness, mg/1 $\mathrm{CaCO}_{3} \mathrm{eq}$.

Chronium

Total, mg/1 Cx

Hexavalent, mg/1 Cr

$\mathrm{COD}, \mathrm{mg} / \mathrm{i} \mathrm{O}_{2}$

Copper, mg/t $\mathrm{Cu}$

Dissr.lved Oxygen, mg/ $1 \mathrm{O}_{2}$

EDTA, mg/ 1 cu eq.

Fecal Coliform Count, MPN/100 ml.

Flow, $\mathrm{ft}^{3} / \mathrm{sec}$

Iron, mg/l Fe

Ni trogen

Total KjeldahI, mg/1 N

Nitrate, $m g / 1 \mathrm{~N}$

oil/Grease, mg/t

$\mathrm{pH}$

Phosphate

Total, mg/1 $\mathrm{p}$

ortho, $\mathrm{mg} / \mathrm{q}$

Solids

Dissolved, $\mathrm{mg} / 1$

suspended, mg/l

Sulfate, $\mathrm{mg} / 1$ so

Temperature, "C

Turbidity, NTO

zinc, mg/l $\mathrm{zn}$

\begin{tabular}{|c|c|c|c|c|c|}
\hline $4 / 3 / 84$ & $4 / 17 / 84$ & $5 / 0 / 84$ & $5 / 22 / 84$ & $6 / 5 / 84$ & $6 / 13 / 84$ \\
\hline $15 \mathrm{a} \cdot \mathrm{m}$. & 10:06 a.me & $11: 27$ a.m. & $10: 35 \mathrm{a}=\mathrm{a}=$ & $10: 15 \mathrm{a}, \mathrm{R}=$ & 10:45 a.m. \\
\hline
\end{tabular}

20

26

0.05

0.01

20

0.01

14

74

0.04

0.56

0.48

0.66

0.86

7.6

0.60

0.22

136

14

16

$13^{\circ} \mathrm{C}$

14

0.047

$\begin{array}{ll}-1 & 36 \\ - & 6\end{array}$

$-$

0.05

0.01

$-$

$-$

$-$

82

0.4

0.30

$-$

$-$

6.9

0.42

0.18

134

10

16

$15^{\circ} \mathrm{C}$

14
10:35 $a+a^{2}$

$=$

$=$

0.05

0.01

-

$-$

$=$

104

0.4

1.6

$-$

$\overline{-}$

7.2

0.055

0.014

142

24

22

$18^{\circ} \mathrm{C}$

7.6
28
1.3

32

0.05

0.01

33.4

0.016

9.6

0.075

175

0.3

0.80

0.60

1.3

0.52

7.0

0. 20

0.05

160

22

28

$18^{\circ} \mathrm{C}$

5.4

0.042
$-$

0.05

0.01

-.

$-$

196

0.5

0.76

$-$

$\overline{-}$

7.1

0.05

0.01

148

16

22

$21^{\circ} \mathrm{C}$

1.7
- 
Table 9 (cont'd)

WATER QUALI'TY MONITORING DATA

Forrestal Campus, Princeton University Station: $\mathbf{B 2}$

Monitoring Station Location: Bee Brook, Lacation B

\section{Parameter}

Alkalinity, $\mathrm{mg} / 1 \mathrm{CaCO}_{3} \mathrm{eq}$.

$\mathrm{BOD}_{5}, \mathrm{mg} / \mathrm{I} \mathrm{O}_{2}$

Calcium Hardness, mg/1 $\mathrm{CaCO}_{3}$ eq.

Chromium

Total, mg/1 Cr

Hexavalent, ing/ $\mathrm{Cr}$

$\mathrm{COD}, \mathrm{mg} / \mathrm{l} \mathrm{O}_{2}$

Copper, mg/1 $\mathrm{Cu}$

Dissolved oxygen, $\mathrm{mg} / 1 \mathrm{O}_{2}$

EDTA, $\mathrm{mg} / 1 \mathrm{Cu}$ eq.

Fecal Coliform Count, MpN/100 ml.

Flow, $\mathrm{ft}^{3} / \mathrm{sec}$

Iron, $\mathrm{mg} / 1 \mathrm{Ee}$

Nitrogen

Total Kjeldahl, $\mathrm{mg} / \mathrm{N}$

Nitrate, mg/1 N

oil/Grease, $\mathrm{mg} / 1$

pH

Phosphate

Total, $\mathrm{mg} / 1 \mathrm{P}$

Solids

Dissolved, mg/1

Suspended, $\mathrm{mg} /$

sulfate, $\mathrm{mg} / \mathrm{i} \mathrm{SO}_{4}$

Temperature, ${ }^{\circ} \mathrm{C}$

Turbidity, NTU

Zinc, mg/1 $2 n$

\begin{tabular}{|c|c|c|c|c|c|}
\hline $7 / 10 / 84$ & $7 / 24 / 84$ & $8 / 7 / 84$ & $8 / 28 / 84$ & $9 / 5 / 84$ & $9 / 18 / 84$ \\
\hline $9: 30$ a.m. & $10: 75 \mathrm{a} \cdot \mathrm{me}$ & $10: 45 \mathrm{a} \cdot \mathrm{m}$. & 10:00 a-m. & 10:00 a-m. & 9:45 a.m. \\
\hline 30 & - & 32 & -- & 20 & $-\infty$ \\
\hline 1.75 & -- & 2.75 & - & 1.1 & - \\
\hline 22 & -- & 26 & -- & 14 & -- \\
\hline 0.05 & 0.05 & 0.05 & 0.05 & 0.05 & 0.05 \\
\hline 0.01 & 0.01 & 0.01 & 0.01 & 0.01 & 0.01 \\
\hline 28 & - & 20 & -- & 12 & -- \\
\hline 0.02 & $=-$ & 0.03 & -- & 0.022 & -- \\
\hline 12 & -- & 14 & -- & 12 & $=$ \\
\hline 0.68 & - & 0.075 & -- & 0.36 & -- \\
\hline 182 & 176 & 184 & 122 & 90 & 82 \\
\hline 0.3 & 0.3 & 0.3 & 0.3 & 0.3 & 0.4 \\
\hline 0.73 & 0.52 & 0.54 & 0.44 & 0.48 & 0.75 \\
\hline 0.68 & - & 0.62 & - & 0.48 & -- \\
\hline 2.90 & -- & 2.72 & - & 2.1 & -- \\
\hline $0.5 b$ & - & 0.45 & -- & 0.44 & $=-$ \\
\hline 7.0 & 7.6 & 7.2 & 7.1 & 7.3 & 7.5 \\
\hline 0.03 & c. $\$ 68$ & 0.065 & 0.06 & 0.04 & 0.82 \\
\hline 0.02 & 0.015 & 0.02 & 0.02 & 0.01 & 0.038 \\
\hline 132 & 122 & 132 & 118 & 120 & 445 \\
\hline 12 & 10 & 14 & 8 & 10 & 38 \\
\hline 16 & 18 & 20 & 16 & 18 & 20 \\
\hline $20^{\circ} \mathrm{C}$ & $21^{\circ} \mathrm{C}$ & $22^{\circ} \mathrm{C}$ & $23^{\circ} \mathrm{C}$ & $18^{\circ} \mathrm{C}$ & $19^{\circ} \mathrm{C}$ \\
\hline 9 & 5 & 4.6 & 1.8 & 1.4 & $8 \cdot 2$ \\
\hline 0.35 & -- & 0.40 & - & 0,038 & -- \\
\hline
\end{tabular}


Table 9 (Cont'd)

WATER QUALITY MONITORING DATA

Forrestal Campus, Princeton University Station: $B 2$

Monitoring station Location: Bee Brook, Location B

\section{Paranetex}

Alkalinity, mg/1 $\mathrm{CaCO}_{3} \mathrm{eq}$. $\mathrm{BOD}_{5}, \mathrm{mg} / 1 \mathrm{O}_{2}$

Calcium Hardness, mg// $\mathrm{CaCO}_{3} \mathrm{eq}$.

Chromium

Total, mg/t $\mathrm{Cr}$

Hoxatalent, ang/1 $\mathrm{Cr}$

$\mathrm{COD}, \mathrm{mg} / 1 \mathrm{O}$

Copper, mg/1 cu

Dìssolved Oxygen, mg/l $\mathrm{O}_{2}$

EDTA, ng/ 1 Cu eq.

Fteal Coliform Count, MPH/100 ml.

Flow, $\mathrm{ft}^{3} / \mathrm{sec}$

iron, $\mathrm{mg} / 1 \mathrm{Fe}$

Nit trogen

Total Kjeldahl, mg/1 N Nitrate, mg/1 N

Oil/Grease, mg/1

pH

Phosphate

Total, mg/i $\mathrm{P}$

ortho, mg/1

Solids

Dissolved, mg/1

Suspended, mg/

Sulfate, $\mathrm{mg} / 1 \mathrm{SO}_{4}$

Temperature, ${ }^{\circ} \mathrm{C}$

Turbidity, NTU

zinc, my/1 zn

\section{$\begin{array}{lllll}10 / 2 / 84 & 10 / 16 / 84 & 11 / 6 / 84 & 11 / 20 / 84 & 12 / 31 / 84\end{array}$ \\ 9:45 a.m. 10:25 a.m. 10:00 a.m. 9:45 a.m. 11:15 a.m.}

$\begin{array}{lllll}22 & -. & 28 & -- & 52 \\ 1.2 & - & 0.65 & -- & 62 \\ 16 & - & 26 & -- & 64\end{array}$

$0.05 \quad 0.05$

0.07

14

0.25

14

0.02

44

0.2

0.32

0.32

I. 41

0.42

6.9

0.03

0.01

0.01

-

$-9$

$-$

48

0.35

0.40

26

0.05

0.01

0.01

14

0.015

14

0.01

40

0.30 .

0.38

0.15

$-$

$-$

7.2

0.48

0.22

7.4

0.04

0.04

0.01

197

134

126

a 16

$15^{\circ} \mathrm{C}$

2

0.02

52

64

0.03

0.0

12

0.03

12

$<0.08$

$<1$

1.1

0.69

0.5

1. 9

$<1$

7.2

0.2

0.1

120

10

22

$8^{\circ} \mathrm{C}$

$16^{\circ} \mathrm{C}$

1.2

0.025

2.5

0.01

210

16

$10^{\circ} \mathrm{C}$

2.5

0.01

0.07 
Table 10

WATER QUALITY MONITORING DATA

Forrestal Campus, Princeton Univergity

station: $\mathrm{C1}$

Monitoring Station Location: Delaware-Raritan Canal

\section{Parameter}

Alkalinity, ng/1 $\mathrm{CaCO}_{3}$ eq.

Calcium Hardness, mg/1 $\mathrm{CaCO}_{3}$ eq.

Chromium

Total, $\mathrm{mg} / \mathrm{C} \mathrm{Cr}$

Hexavalent, mg/1 Cr

Copper, ing/ $\mathrm{Cu}$

EDTA $\mathrm{mg} / 1 \mathrm{Cu}$ eq

Iron, $\mathrm{mg} / 1 \mathrm{Fe}$

Nitrate, $\mathrm{mg} / \mathrm{t} \mathrm{N}$

Oil and Grease, mg/l

plit

Phosphate

Total, mg/l

Ortho, mg/1 p

Solids

Dissolved

Suspended, mg/1

Sulfate, mg/1 $\mathrm{SO}_{4}$

Turbidity, NTU

zinc, $\mathrm{mg} / 1 \mathrm{zm}$

\begin{tabular}{|c|c|c|c|c|c|}
\hline $1 / 10 / 84$ & $2 / 7 / 84$ & $3 / 6 / 84$ & $4 / 3 / 84$ & $5 / 8 / 84$ & $6 / 5 / 84$ \\
\hline $9: 37$ a.m. & $10: 19 \mathrm{a}+\mathrm{m}_{\mathrm{a}}$ & $10: 11 \mathrm{am}$. & $11: 30 \mathrm{a}, \mathrm{m}_{0}$ & $11: 30 \mathrm{a} \cdot \mathrm{m}$. & 10:45 a.ta. \\
\hline 26 & 33 & 28 & 25 & 26 & 26 \\
\hline 34 & 38 & 32 & 28 & 30 & 28 \\
\hline 0.05 & 0.05 & 0.05 & 0.05 & 0.05 & 0.05 \\
\hline 0.01 & 0.0 .1 & 0.01 & 0.01 & 0.01 & 0.01 \\
\hline 0.010 & 0.037 & 0.022 & 0.01 & 0.015 & 0.01 \\
\hline 0.025 & 0.020 & 0.02 & 0.022 & 0.01 & 0.02 \\
\hline 0.18 & 0.29 & 0.39 & 0.56 & 0.55 & 0.70 \\
\hline 1.57 & 2,43 & 1.76 & 1.22 & 9.6 & 1.74 \\
\hline 0.10 & 0.12 & 1.2 & 1.95 & 2.3 & 0.20 \\
\hline $7 \cdot 3$ & 7.1 & 7.0 & 7.5 & 7.0 & 7.5 \\
\hline 0.060 & 0.09 & 0.056 & 0.065 & 0.05 & $0 . \$ 6$ \\
\hline 0.055 & 0.06 & 0.032 & 0.022 & 0.02 & 0.047 \\
\hline 133 & 102 & 126 & 140 & 108 & 142 \\
\hline 12 & 6 & 8 & 8 & 20 & 15 \\
\hline 20 & 24 & 26 & 18 & 20 & 22 \\
\hline 6 & 7 & .8 .8 & 22 & 28 & a. 2 \\
\hline 0.031 & 0.032 & 0.043 & 0.022 & 0.028 & 0.032 \\
\hline
\end{tabular}


Table 10 (Cont'd)

WATER QUALITY MONITORING DATA

Forrestal Campus, Princeton University

station: $c 1$

Monitoring station Location: Delaware-Raritan Canal

\section{Parameter}

Alkalinity, mg/ $\mathrm{CaCO}_{3}$ eq.

Calcium Hardness, mg/i $\mathrm{CaCO}_{3} \mathrm{eq}$.

Chromium

rotal, mg/1 Cr

Hexayalent, mg/t cr

Copper, mg/l $\mathrm{Cu}$

EDTA mg/1 Cu eq.

Iron, ing/1 $\mathrm{Fe}$

Nitrate, $\mathrm{mg} / \mathrm{l} \mathrm{N}$

oil and Grease, $\mathrm{mg} / \mathrm{l}$

$\mathrm{pH}$

Phosphate

Total, mg/1 P

ortho, ag/1 $P$

Solids

Dissolved

Suspended, mg/ 1

sulfate, $\mathrm{mg} / \mathrm{i} \mathrm{SO}_{4}$

Turbidity, NTU

zinc, mg/1 $\mathrm{zn}$
$7 / 10 / 84$

0.05

0.01

0.015

0.02

0.35

0.22

0.35

$7+1$

0.026

0.01

$16 \theta$

14

24

18

0.036
11:00 a.m.

2B

34

0.05

$9 / 5 / 84$

9:15 a.m.

26

30

0.05

0.01

$0.01 \mathrm{~B}$

0.01

0.015

0.55

1.20

0.42

6.8

0.03

0.01

148

18

20

6.3

0.034

0.012

0.22

0.76

0.32

7.2

0.025

0.01

120

12

22

3.9

0.03
$10 / 2 / 84$
25
30

0.07

0.01

0.0

0.39

1.7

0.30

7.0

0.05

0.02

116

7

18

2.5

0.01
$11 / 6 / 84$

9.00 a.m.

30

26

0.05

0.01

0.015

$12 / 31 / 84$

$11: 30$ a.m.

16

60

$<.01$

$<.01$

$<.08$

.70

.97

2.4

0.38

0.80

0.32

6.9

1.0

0.06

0.015

.12

0.08

743

15

20

4.5

0.022 
Table 11

WATER QUALITY MONITORING DATA

Forrestal Campus, Princeton Univarsity Station: D1

Monitoring station Location: Drainage Ditch \#5, Lacation A

\section{Parameter}

Alkalinity, $\mathrm{mg} / 1 \mathrm{CaCO}_{3} \mathrm{eq}$.

$\mathrm{BOD}_{5}, \mathrm{lng} / 1 \mathrm{O}_{2}$

Calcium Hardness, mg/1 $\mathrm{CaCO}_{3} \mathrm{eq}$.

Chromium

Total, mg/l cr

Hexavalent, mg/ $\mathrm{Cr}$

$\mathrm{COD}, \mathrm{mg} / \mathrm{l} \mathrm{O}$

Copper, mg/1 $\mathrm{Cu}$

Dissolved Oxygen, mg/7 $O_{2}$

EDTA, mg/l Cu eq.

Fecal coliform Count, MPN/100 ml.

Flow, $\mathrm{ft}^{3} / \mathrm{sec}$

Iron, $\mathrm{mg} / 1 \mathrm{Fe}$

Nitrogen

Total Kjeldahl, mg/l

Nitrate, $\mathrm{m}_{3} / 1 \mathrm{~N}$

oil/Grease, $\mathrm{mg} / 1$

pH

Phosphate

Total, mg/1 p

ortho, $m g / 1$ P

solids

Dissolyed, mg/1

Suspended, mg/1

sulfate, $\mathrm{mg} / 1 \mathrm{So}_{4}$

Temperature, ${ }^{\circ} \mathrm{C}$

lurbidity, NTU

zinc, mg/g an

\begin{tabular}{|c|c|c|}
\hline $1 / 10 / 84$ & $7 / 24 / 84$ & $2 / 7 / 8$ \\
\hline $9.08 \mathrm{a} . \mathrm{m}$. & $9: 30 \mathrm{om} \cdot$ & $9: 44$ \\
\hline $\begin{array}{l}30 \\
4.6\end{array}$ & -- & $\begin{array}{l}44 \\
1.6\end{array}$ \\
\hline 24 & $=-$ & 42 \\
\hline 0.05 & 0.05 & 0.05 \\
\hline 0.01 & 0.01 & 0.01 \\
\hline 6.5 & $-\infty$ & 9.8 \\
\hline 0.10 & $m$ & 0.02 \\
\hline 9.2 & $\omega$ & 10.6 \\
\hline 0.025 & $-\infty$ & 0.07 \\
\hline-- & -- & -- \\
\hline 1.0 & 0.9 & 0.5 \\
\hline 0.57 & 0.53 & 0.30 \\
\hline- & & \\
\hline 0.29 & & \\
\hline 1.33 & & \\
\hline 0.28 & -- & 4.0 \\
\hline 7.1 & 7.0 & 7.2 \\
\hline 0.096 & 0.085 & 0.32 \\
\hline 0.050 & 0.040 & 0.15 \\
\hline 152 & 140 & 112 \\
\hline 18 & 16 & 13 \\
\hline 16 & 15 & 40 \\
\hline $9^{\circ} \mathrm{C}$ & $10^{\circ} \mathrm{C}$ & $8^{\circ} \mathrm{C}$ \\
\hline 10 & 9 & $: 3$ \\
\hline 0.032 & - & 0.05 \\
\hline
\end{tabular}

$2 / 21 / 84$

9:15 a.m.

$3 / 6 / 84$

$9: 36$ a.m.

32
1.6

33

0.05

0.01

12

0.022

12

0.018

1.0

0.46

12

7.1

6.9

0.16

0.032

162
16
22
$8^{\circ} \mathrm{C}$
13
0.04

132
14
36
$10^{\circ} \mathrm{C}$
14
$=-$

0.072

$3 / 20 / 84$

9:53 a.m.

$\overline{-}$

0.05

0.01

$+$

$\rightarrow$

$-$

.6

1.3

7.

106

14

25

$9^{\circ} \mathrm{C}$

17 
Table 11 (Cont'd)

WATER QUALITY MONITORING DATA

Forrestal Campus, Princeton Univeraity Station: DI

Monitoring Station Location: Drainage Ditch $\$ 5$, Location A

Parametor

Altalinity, mg/1 $\mathrm{CaCO}_{3} \mathrm{eq}$.

$\mathrm{BOD}_{5}, \mathrm{mg} / \mathrm{l} \mathrm{O}_{2}$

Calcium Hardness, mg/1 $\mathrm{CaCO}_{3}$ eq.

chronium

Total, mg/1 $\mathrm{cr}$

Hexavalent, mg/A $\mathrm{Cr}$

COD, $\mathrm{mg} / 1 \mathrm{O}$

Copper, mg/1 Cu

Dissolved Oxygen, $\mathrm{mg} / 1 \mathrm{O}_{2}$

EDTA, $\mathrm{mg} / \mathrm{l}$ Cu eq.

Fecal Coliform Count, MPN/100 ml.

Flow, $\mathrm{ft}^{3} / \mathrm{sec}$

Iron, $\mathrm{mg} / 1 \mathrm{Fe}$

Nitrogen

Total Kjeldahl, $\mathrm{mg} / 1 \mathrm{~N}$

Nitrate, $\mathrm{mg} / \mathrm{N}$

oil/Grease, mg/1

$\mathrm{pH}$

Phosphate

Total, $\mathrm{mg} / \mathrm{l} \mathrm{P}$

Ortho, mg/l $P$

Solids

Dissolved, my/l

sispended, $\mathrm{mg} / 1$

Sulfate, $\mathrm{mg} / 1 \mathrm{SO}_{4}$

Temperature, ${ }^{\circ} \mathrm{C}$

Turbidity, NTU

zinc, $\mathrm{mg} / \mathrm{Zn}$
$4 / 3 / 84$

$4 / 17 / 84$

$5 / 8 / 84$

11:15 a.m.

11:02 a+m. 9:52 ה.m.

.6

36

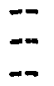

0.05

0.01

10

0.024

11

0.033

$-5$

0.68

0.46

1.40

1.2

7.2

0.75

0.35

130

22

22

$12^{\circ} \mathrm{C}$

11

0.044
0.05

0.01

$\rightarrow$

$-$

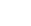

1.0

0.56

7

7.4

0.68

0.32

132

32

20

$14^{\circ} \mathrm{C}$

15

-.
32
1.4

36

10:15 2.

28

32

\subsection{5}

0.01

9.8

0.01

9.4

0.04

-

0.5

0.72

0.65

7. 6

0.055

0.039

128

22

20

$15^{\circ} \mathrm{C}$

36

0.052

\section{$-$}

0.05

0.01

-

$-$

$-$

0.5

0.98

7.2

.52

7.5

0.049

0.043

20

24

18

$19^{\circ} \mathrm{C}$

2.3
$5 / 22 / 84$

$6 / 5 / 84$

7.2

0.096

0.078

142

$19^{\circ} \mathrm{C}$

7.5

0.038

$6 / 13 / 84$

10: $15 \mathrm{a} . \mathrm{m}$.

$\overline{-}$

0.05

0.01

-

$-$

$-$

$-$

0.6

0.62 
Table 11 (Cont'a)

WATER QUALITY MONITORING DA'A

Forrestal Campus, Princeton Univergity

Station: DI

Monitoring station Location: Drainage Ditch $\$ 5$, Location A

\section{Parameter}

Alkalinity, mg/r $\mathrm{CaCO}_{3} \mathrm{eq}$.

$\mathrm{BOD}_{5}, \mathrm{mg} / 1 \mathrm{O}_{2}$

Calcium Hardness, $\mathrm{mg} / 1 \mathrm{CaCO}_{3} \mathrm{eq}$.

Chromium

Total, mg/l $\mathrm{Cr}$

Hexavalent, mg/1 cr

$\mathrm{COD}, \mathrm{mg} / 1 \mathrm{O}$

Copper, mg/ $\mathrm{Cu}$

Dissolved oxygen, mg/1 $\mathrm{o}_{2}$

EDTA, $\mathrm{mg} / \mathrm{l} \mathrm{Cu} \mathrm{eq}$.

Fecal Coliform Count, MPN/100 ml.

Flow, $\mathrm{ft}^{3} / \mathrm{gec}$

Iron, $\mathrm{mg} / 1 \mathrm{Fe}$

Nitrogen

Total kjeldahl, mg/l iv

Nitrate, $\mathrm{mg} / 1 \mathrm{~N}$

oil/Grease, mg/1

$\mathrm{pH}$

Phosphate

Total, mg/1 $\mathrm{p}$

ortho, mg/1 $\mathrm{P}$

Solids

Dissolved, $\mathrm{mg} / 1$

suspended, mg/1

sulfate, $\mathrm{mg} / \mathrm{t}$ so

Temperature, ${ }^{\circ} \mathrm{C}$

Turbidity, NIu

zinc, $\mathrm{mg} / \mathrm{z} \mathrm{zn}$
$7 / 10 / 84$

B:45 a.m

30

2.5

3.4

0.05

0.01

8

0.028

10

0.03

0.3

0.55

0.36

1.28

0.30

7.0

0.86
0.38

152

18

18

$22^{\circ} \mathrm{C}$

9

0.042

\section{$7 / 24 / 84$}

9:30 a.m.

$\sim$

$-$

0.05

0.01

a-

$-$

$-$

0.4

0.64

$-$

$-$

7.3

0.72

0.42

132

16

12

$25^{\circ} \mathrm{C}$

8.6

$-$
8/7/84

$10: 15 \mathrm{a} \cdot \mathrm{m}$.

34

1.9

34

0.05

0.01

9

0.025

11

0.04

0.4

0.48

0.42

1.84

0.60

7.1

0.076

0.03

126

20

22

$24^{\circ} \mathrm{C}$

10

0.044

\section{B/28/84}

9:15 a.m.

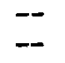

$-$

0.05

0.01

$-$

$-$

$-$

0.3

0.60

$-$

$-$

7.0

0.35

0,02

115

18

16

$23^{\circ} \mathrm{C}$

2.1

$-$
9/5/84

9:30 a.m.

32

0.75

30

0.05

0.01

12

0.22

12
0.025

0.025

0.3

0.40

0.32

1.40

0.40

7.0

0.40

0.025

120

16

18.

$19^{\circ} \mathrm{C}$

1.6

0.04
$9 / 18 / 84$

9:15 a.m.

$-$

$-$

0.05

0.01

--

$-$

--

0.5

0.65

$=$

$-$

7.2

0.95

0.04

360

30

26

$20^{\circ} \mathrm{C}$

$4+2$ 
Table 11 (ront'd)

HATER QUALITY MONITORING DATA

Forregtal Campus, Princeton University Station: D1

Monftoring Station Location: Drainage Ditch \#5, Location A

\section{Parameter}

Alkalinity, mg/l $\mathrm{CaCO}_{3} \mathrm{eq}$.

$\mathrm{BOD}_{5}, \mathrm{mg} / \mathrm{l} \mathrm{O}_{2}$

calcium Haraness, mg/1 $\mathrm{CaCO}_{3} \mathrm{eq}$ Chromi in

$$
\begin{aligned}
& \text { Total, mg/1 } \mathrm{Cr} \\
& \text { Hexavalent, mg/1 Cr }
\end{aligned}
$$

Cop, $\mathrm{mg} / \mathrm{h} \mathrm{O}_{2}$

Copper, ing/1 $\mathrm{Cu}$

Dissolved oxygen, mg/1 $o_{2}$

EDTA, mg/1 Cu eq.

Fecal Coliform Count MPN/100 ml.

Flow, $\mathrm{ft}^{3} / \mathrm{sec}$

Iron, $\mathrm{mg} / 1 \mathrm{Fe}$

Nitrogen

Total Kjeldahl, mg/1 N

Nitrate, mg/1 $\mathrm{N}$

Oil/Grease, mg/1

pH

Phosphate

Total, mg/1 P

Ortho, $\mathrm{mg} / 1 \mathrm{P}$

Solids

Dissolved, $\mathrm{mg} / 1$

Susponded, mg/1

Sulfate, $\mathrm{mg} / 1 \mathrm{SO}_{4}$

Temperature, ${ }^{\circ} \mathrm{C}$

Turbidity, NTU

Zins, mg/1 zn

\section{$10 / 2 / 84$}

$9: 55 \mathrm{a}+\mathrm{m}$.

26

1.2

30

0.05

0.01

12

0.02

14

0

0.3

0.32

0.70

3.08

0.42

6.6

0.05

0.03

120

8

22

$77^{\circ} \mathrm{C}$

1.9

0.02

0.30

$-$

$-$

7.1

0.08

0.04

126

6

20

$17^{\circ} \mathrm{C}$

1.4

0.02

\section{$11 / 6 / 84$}

$9: 15$ a.m.

30

0.85

32

0.05

0.01

10

0,022

12

0.03

0.3

0.3

0.25

0.60

0.22

7.0

0.06

0.03

220

25

22

$15^{\circ} \mathrm{C}$

2.9

0.02
$11 / 20 / 84$

:00 a.ra. 10:30 a.m.

20

$-$

$+$

$<2$

40

< 0.01

$<0.01$

28

0.04

15

0.08

$<1$

4

0.64

0.7

2. 1

$<1$

6.8

0.2

0.05

0.01

0.1

10

20

$11^{\circ} \mathrm{C}$

3.8

0.02

85

30

16

$8^{\circ} \mathrm{C}$

22

0.07 
WATER OUALITY MONITORING DATA

Forrestal Campus, Princeton University

$$
\text { Station: } 02
$$

Monitoring Station Location: Drainage Ditch \#5, Location $B$

Parameter

Alkallni $\leftleftarrows y$

$\mathrm{BOD}_{5}, \mathrm{mg} / \mathrm{l} \mathrm{O}_{2}$

Calcium Hardness, mg/1 $\mathrm{CaCO}_{3}$ eq.

Chromium

Total, $\mathrm{mg} / 1 \mathrm{cr}$

Hexavalent, $\mathrm{mg} / 1 \mathrm{Cr}$

CoD, $\mathrm{mg} / 1 \mathrm{O}$

Copper, mg/1 $\mathrm{Cu}$

Dissolved Oxygen, mg/l $\mathrm{O}_{2}$

EDTA, mg/1 Cu eq.

Feaal Coliform Count, MPN/100 ml.

Flow, $\mathrm{ft}^{3} / \mathrm{sec}$

Iron, $\mathrm{mg} / 1 \mathrm{Fe}$

Nitrogen

Total Kjeldahl, mg/i N

Nitrate, $m g / 1 \mathrm{~N}$

Oil/Grease, mg/i

pH

Phosphate

Total, mg/l $\mathrm{p}$

Ortho, mg/l p

Sollds

Dissolved, mg/

Suspended, mg/

Sulfate, $m g / 1 \mathrm{SO}_{4}$
Temperature, ${ }^{\circ} \mathrm{C}$

Turbldity, NTU

zinc, mg/l zn

\section{$1 / 10 / 84$ \\ 9:15 a.m.}

38

5.0

38

0.05

0.01

10.5

0.018

10.8

0.040

0.30

0.45

0.43

1.66

0.92

7.0

0.22

0.045

165

25

18

$10^{\circ} \mathrm{C}$

7

0.070

\section{$1 / 24 / 84$}

9:44 a.m.

$--$

$-$

0.05

0.01

--

$-$

$+$

--

0.30

0.48

$-$

$-$

7.0

0.33

0.04

154

22

14

$10^{\circ} \mathrm{C}$

$B$

--
$2 / 7 / 64$

9:53 a.m.

42
1.5

38

0.05

0.01

6.2

0.042

11.2

0.07

.

0.55

0.52

1.55

3. 2

7.1

0.32

0.21

118

12
18

18

10

0.032
$2 / 21 / 84$

9:30 a.1n.

$-$

0.05

0.01

--

$-$

$-$

$-$

0.56

$-$

$-$

7.2

0.36

a. 18

126

14

16

$9^{\circ} \mathrm{C}$

12
$3 / 6 / 84$

9:45 a.m.

$3 / 20 / 84$

10:03 a.m.

三

0.05

0.01

--

$-$

$-$

.3

0.42

-

0.18

1.86

7.2

6.9

0.16

0.10

126

14

28

$9^{\circ} \mathrm{C}$

17

0.042 
Table 12 (Cont'd)

WATER QUALITY MONITORING DATA

Forrestal Campus, Princeton University

Station: D2

Mondtoring station Location: Drainage Ditch 45, Location B

Parameter

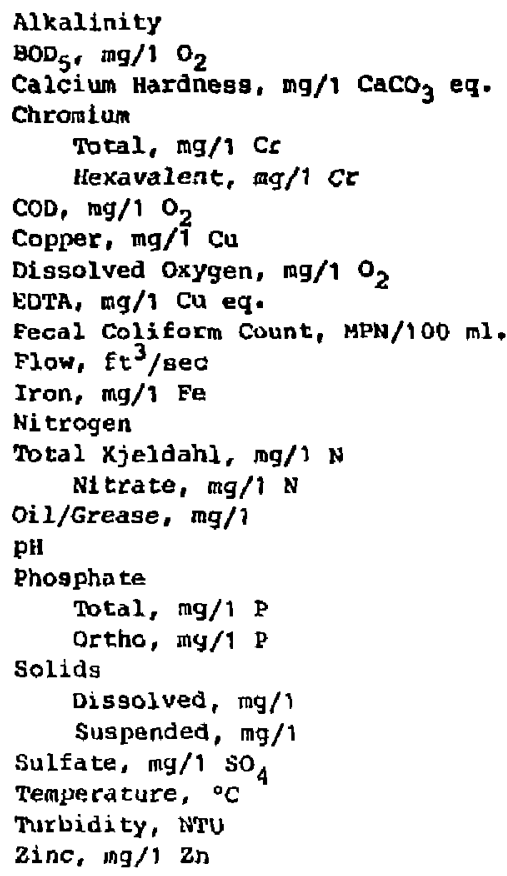

\begin{tabular}{|c|c|c|c|c|c|}
\hline $\begin{array}{l}4 / 3 / 84 \\
11: 15 \text { a.17. } \\
\end{array}$ & $\begin{array}{l}4 / 17 / 84 \\
10: 01 \mathrm{a} . \mathrm{m} . \\
\end{array}$ & $\begin{array}{l}5 / 8 / 84 \\
11: 20 \text { a.m. } \\
\end{array}$ & $\begin{array}{l}5 / 22 / 84 \\
10,25 \mathrm{a} . \mathrm{m} .\end{array}$ & $\begin{array}{l}6 / 5 / 84 \\
9: 30 \text { a.r.n. } \\
\end{array}$ & $\begin{array}{l}6 / 13 / 84 \\
10: 25 \text { a.m. } \\
\end{array}$ \\
\hline 36 & -- & 34 & - & 30 & $\infty$ \\
\hline 3.2 & -- & 1.2 & - & 1.2 & - \\
\hline 36 & - & 32 & - & 30 & - \\
\hline 0.05 & 0.05 & 0.05 & 0.05 & 0.05 & 0.05 \\
\hline 0.01 & 0.01 & 0.01 & 0.01 & 0.01 & 0.01 \\
\hline 14 & -- & 11 & -- & 11 & -- \\
\hline 0.022 & $\sim$ & 0.024 & - & 0.026 & - \\
\hline 10.3 & -- & 11.4 & - & 10.5 & -- \\
\hline 0.065 & $\infty$ & 0.065 & - & 0.065 & -- \\
\hline-- & -- & $-\infty$ & - & - & - \\
\hline 0.03 & 0.04 & 0.4 & 0.4 & 0.3 & 0.5 \\
\hline 1.2 & 0.42 & 0.65 & 1.34 & 0.88 & 0.80 \\
\hline 0.68 & - & 0.55 & $-\infty$ & 0.40 & - \\
\hline 1.25 & -- & 0.72 & - & 1.24 & - \\
\hline 1.6 & -- & 0.46 & - & 0.46 & -- \\
\hline 6.8 & 6.8 & 7.2 & 7.0 & 6.9 & 7.0 \\
\hline 0.075 & 0.20 & 0.045 & 0.14 & 0.085 & 0.075 \\
\hline 0.055 & 0.085 & 0.025 & 0.044 & 0.65 & 0.042 \\
\hline 132 & 134 & 148 & 173 & 158 & 163 \\
\hline 22 & 36 & 58 & 5 & 24 & 19 \\
\hline 24 & 22 & 22 & 20 & 20 & 24 \\
\hline $14^{\circ} \mathrm{C}$ & $15^{\circ} \mathrm{C}$ & $15^{\circ} \mathrm{C}$ & $19^{\circ} \mathrm{C}$ & $19^{\circ} \mathrm{C}$ & $21^{\circ} \mathrm{C}$ \\
\hline 15 & 17 & 28 & 1.7 & 6.5 & 1.7 \\
\hline 0.038 & $-=$ & 0.64 & - & 0.032 & $=$ \\
\hline
\end{tabular}


Table 12 (Cont'd)

WATER QUALITY MONITCRING DATA

Eorrestal Campug, Princeton University Station: 02

Moni toring station Location, Drainage Ditch $\$ 5$, Location B

\section{Parameter}

Alkalinity

$\mathrm{BOD}_{5}, \mathrm{ng} / 1 \mathrm{O}_{2}$

Calcium Hardness, mg/1 $\mathrm{CaCO}_{3} \mathrm{eq}$. Chromium

Total, mg/1 Cr

Hexavalent, mg/1 $\mathrm{Cr}$

$\mathrm{COD}, \mathrm{mg} / 1 \mathrm{O}$

Copper, mg/1 $\mathrm{Cu}$

Dissolved oxygen, mg/l $\mathrm{O}_{2}$

EDTA, mg/1 Cu eq

Fecal Coliform Count, MPN/100 ml.

Flow, $\mathrm{ft}^{3} / \mathrm{sec}$

Iron, $\mathrm{mg} / \mathrm{i}$ Ee

Ni trogen

Iotal Kjeldahl, mg/l N

Nitrate, $\mathrm{mg} / 1 \mathrm{~N}$

oil/Grease, mg/l

pll

Bhosphate

Total, mg/t $p$

ortho, $\mathrm{mg} / \mathrm{l} \mathrm{P}$

Solidg

Dissolved, ing / 1

Suspended, ing $/ 1$

Sulfate, $\mathrm{mg} / \mathrm{l} \mathrm{SO}_{4}$

Teimperature, ${ }^{\circ} \mathrm{C}$

Iurbidity, NTU

2lne, ing/1 Zn

\begin{tabular}{|c|c|c|}
\hline $\begin{array}{l}7 / 10 / 84 \\
9: 00 \text { a.m. }\end{array}$ & $\begin{array}{l}7 / 24 / 84 \\
9: 45 \mathrm{a} . \mathrm{m} .\end{array}$ & $\begin{array}{l}8 / 7 / 84 \\
10: 30\end{array}$ \\
\hline 28 & -- & 32 \\
\hline 2.75 & $-\infty$ & 2.90 \\
\hline 36 & - & 34 \\
\hline 0.05 & 0.05 & 0.05 \\
\hline 0.01 & 0.01 & 0.01 \\
\hline 10 & - & 12 \\
\hline 0.026 & - & 0.02 \\
\hline 11 & - & 10 \\
\hline 0.028 & - & 0.036 \\
\hline-- & -- & - \\
\hline 0.3 & 0.4 & 0.3 \\
\hline 0.60 & 0.32 & 0.54 \\
\hline 0.40 & -- & 0.52 \\
\hline 1.76 & -- & 2.28 \\
\hline 0.28 & - & 0.58 \\
\hline 7.0 & 7.3 & 7.2 \\
\hline 0.130 & 0.06 & 0.72 \\
\hline 0.32 & 0.036 & 0.24 \\
\hline 160 & 155 & 150 \\
\hline 22 & 24 & 24 \\
\hline 24 & 22 & 22 \\
\hline $22^{\circ} \mathrm{C}$ & $23^{\circ} \mathrm{C}$ & $22^{\circ} \mathrm{i}$ \\
\hline 12 & 5.9 & 5 \\
\hline 0.04 & -- & 0.036 \\
\hline
\end{tabular}

$8 / 28 / 84$

9:30 a.m.

$-$

$\rightarrow$

$m$

0.05

0.01

$-$

$-$

$-$

$-$

0.3

0.48

$--$

$-$

7.9

$9 / 5 / 84$

9:45 a.m.

$9 / 18 / 84$

9:30 a:mt

0.90

$-$

32

0.05

0.01

12

0.016

12

0.03

0.4

0.40

0.05

0.01

$-$

$\overline{-}$

0.45

1.90

0.30

7.2

0.36

0.18

0.15

132

16

20

$19^{\circ} \mathrm{C}$

1.5

0.03
$-$

0.75

0.80

$-$

$-$

$7 \cdot 4$

0.90

\section{st}

0.035

500

42

24

$19^{\circ} \mathrm{C}$

H. 5 
WATER QUALITY MONITORING DATA

Forregtal Campus, princeton University

Station: 02

Monitoring Station Location: Dralnage pitch "5, Location

Parameter

Alkalinity

$\mathrm{BOD}_{5}, \mathrm{mg} / 1 \mathrm{O}_{2}$

Calcium Hardness, mg/l $\mathrm{CaCO}_{3} \mathrm{eq}$ Chromium

$$
\text { Total, mg/l cr }
$$

Hexavalent, og/ $\mathrm{cr}$

$\mathrm{COD}, \mathrm{mg} / 1 \mathrm{O}$

Copper, mg/t $\mathrm{Cu}$

Dissolved Oxygen, $\mathrm{mg} / 1 \mathrm{O}_{2}$

EDTh, mg/1 Cu eq.

Fecal Coliform Count, MPH/100 ml,

Flow, $\mathrm{Et}^{3} / \mathrm{sec}$

Iron, $\mathrm{mg} / 1 \mathrm{Fe}$

Ni trogen

Total Kjeldahl, mg/j

Nitrate, mg/1 N

Oil/Grease, $\mathrm{mg} / 1$

$\mathrm{pH}$

Phosphate

$$
\text { Total, mg/1 } P
$$

ortho, mg/1 P

Solids

Dissolved, mg/1

Suspended, $\mathrm{mg} / 1$

sulfate, rig/ $\mathrm{SO}_{4}$

Temperature, ${ }^{\circ} \mathrm{C}$

'lurbiảity, NIU

zinc, mg/l zn

\section{$10 / 2 / 84$ \\ 9:15 a.m.}

30

1.1

29

0.05

0.01

13

0.02

14

0

0.3

0.28

0.20

0.88

0.40

6.8

0.04

0.025

116

20

$16^{\circ} \mathrm{C}$

1.9

0.015
$10 / 16 / 84$

10:05 a.m.

$-$

$-$

0.05

0.01

$-$

$-\infty$

$-$

0.55

0.32

$--$

$\rightarrow$

7.2

0.05

0.02

124

4

22

$17^{\circ} \mathrm{C}$

1.1

0.01

\section{$11 / 6 / 84 \quad 11 / 20 / 84$}

$9 \div 30 \mathrm{a} \cdot \mathrm{m}_{*} \quad 9: 15 \mathrm{a}+\mathrm{m}$

28

0.70

28

$-$

$-$

0.05

0.05

0.01

12

0.02

12

0.015

- 0.3

0.40

0.20

0.50

0. 28

7.2

0.06

0.02

212

22

20

$15^{\circ} \mathrm{C}$

4.2

0.02
0.01

--

$=$

$-$

-

0.3

0.46

$-$

$-$

7.3

0.04

0.01

225

11
18

18

2. 8

0.02
$12 / 31 / 84$

10:45 a.m.

$<2$

$6 B$

0.02

$<.01$

42

0.04

13

$<0.08$

$<1$

1

1.1

0.7

2.2

$<1$

6.9

0.15

0.01

110

98

22

$10^{\circ} \mathrm{C}$

27

0.09 
Table 13

WATER QUALITY MONITORING DATA

Forrestal Campus, Irinceton University

Station: E1

Monitoring station Location: On site Elizabethtown potable water

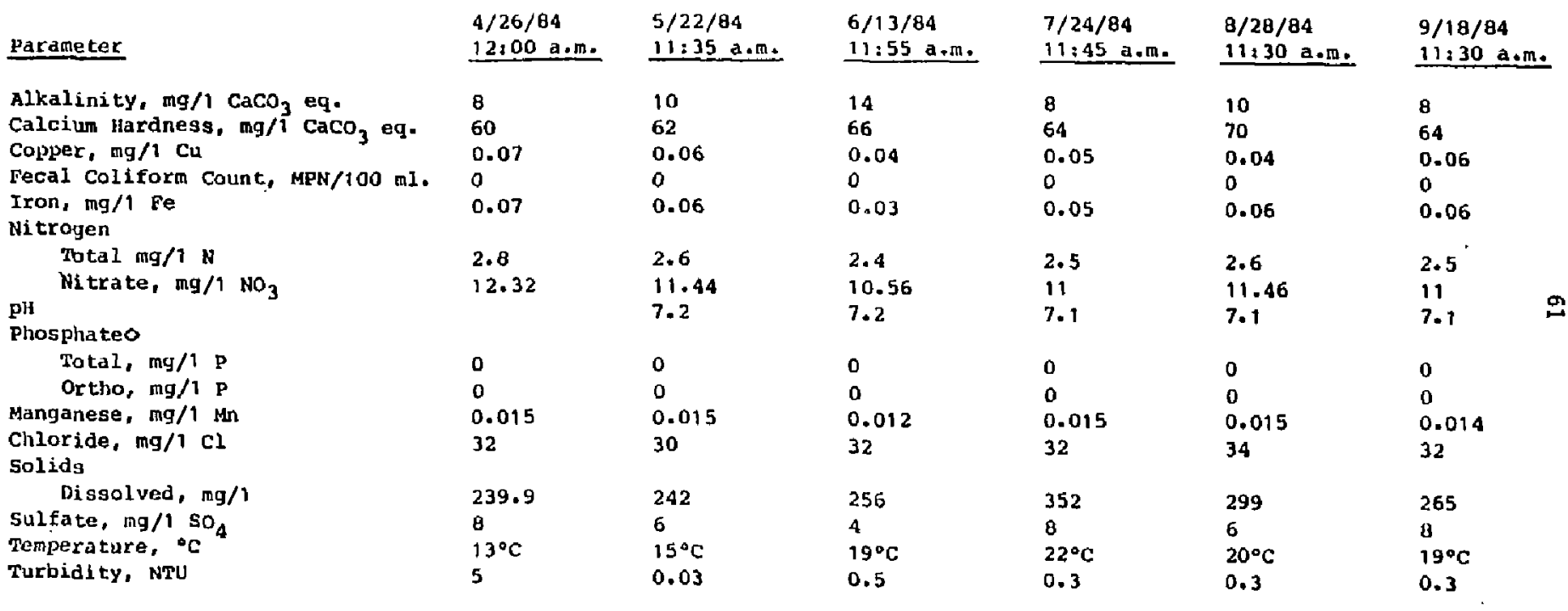


Table 13 (Cont'd)

WATER QUALITY MONITORING DATA

Forrestal Campus, Princeton University Station: E1

Monitoring Station Lacation: On Site Elizabethtown potable water

\section{parameter}

Alkalintty, mg/1 $\mathrm{CaCO}_{3} \mathrm{eq}$.

Calcium fiardness, mg/i $\mathrm{CaCO}_{3} \mathrm{eq}$.

Copper, mg/1 Cu

Fecal Coliform Count, MPN/100 ml.

Iron, $m g / 1 \mathrm{Fe}$

Hitrogen

Total mg/i $\mathrm{H}$

Nitrato, mg/1 $\mathrm{NO}_{3}$

$\mathrm{pH}$

Phosphateo

Total, mg/l $\mathrm{P}$

ortho, mo/1

Manganese, $\mathrm{mg} / \mathrm{l}$ Mn

Chloride, $\mathrm{mg} / \mathrm{l} \mathrm{Cl}$

Solids

Dissolved, mg/ 1

Sulfate, $\mathrm{mg} / 1 \mathrm{so}$

Temperature, ${ }^{\circ} \mathrm{C}$

Turbidity, NTU
$10 / 16 / 84 \quad 11 / 20 / 84$

$11: 35 \mathrm{a} . \mathrm{m} . \quad 11: 15 \mathrm{a} . \mathrm{m}$

$\begin{array}{ll}8 & 10 \\ 60 & 62 \\ 0.05 & 0.04 \\ 0 & 0 \\ 0.04 & 0.04 \\ 2.4 & \\ 10.56 & 2.1 \\ 7.0 & 9.25 \\ 0.0 & 7.1 \\ 0.0 & 0 \\ 0.01 & 0 \\ 34 & 0.01 \\ 240 & 36 \\ 10 & \\ 17^{\circ} \mathrm{C} & 303 \\ 0.3 & 12 \\ & 15^{\circ} \mathrm{C} \\ & 0.3\end{array}$


Table 14

WATER QUALITY MONITOHING DATA

Forrestal Campus, Princeton Untvergity

Station: $\mathrm{Pl}$

Monitoring station Location: Schalk Station Creek, 0.75 miles, S.E.

\begin{tabular}{|c|c|c|c|c|c|c|}
\hline & $4 / 26 / 84$ & $5 / 22 / 84$ & $6 / 13 / 67$ & $7 / 24 / 84$ & $8 / 28 / 84$ & $9 / 18 / 84$ \\
\hline Parameter & $11: 15 \mathrm{a} \cdot \mathrm{m}$. & $10: 55 \mathrm{a}=\mathrm{m}$. & $11 \div 00 \mathrm{a} \cdot \mathrm{m}$. & $10: 45 \mathrm{a} . \mathrm{m}$. & 10:30 a.m. & 10:30 a-m. \\
\hline Alkalinity, mg// $\mathrm{CaCO}_{3} \mathrm{eq}$ & 6 & B & 12 & 10 & $B$ & 8 \\
\hline $\begin{array}{l}\text { Caleium Hardness, } \mathrm{mg} / \mathrm{i} \mathrm{CaCO}_{3} \text { eq. } \\
\text { Chromium }\end{array}$ & 20 & 18 & 20 & 18 & 20 & 22 \\
\hline Total, $\mathrm{mg} / 1 \mathrm{Cr}$ & 0.05 & 0.05 & 0.05 & 0.05 & 0.05 & 0.05 \\
\hline Hexavalent, $\mathrm{mg} / 1 \mathrm{cr}$ & 0.01 & 0.01 & 0.01 & 0.01 & 0.01 & 0.01 \\
\hline Copper, mg/ $1 \mathrm{Cu}$ & 0.04 & 0.06 & 0.00 & 0.05 & 0.06 & 0.04 \\
\hline $\begin{array}{l}\text { Iron, mg/1 } \mathrm{Fe} \\
\text { Nitrogen }\end{array}$ & 0.35 & 0.56 & 0.62 & 0.44 & 0.40 & 0.36 \\
\hline Nitrate, mg/ $1 \mathrm{~N}$ & 0.7 & 0.66 & 0.78 & 0.72 & 0.60 & 0.74 \\
\hline $\begin{array}{l}\text { Oil/Grease, mg/1 } \\
\text { plt }\end{array}$ & $\begin{array}{l}0.68 \\
7.7\end{array}$ & $\begin{array}{l}0.72 \\
7.2\end{array}$ & $\begin{array}{l}0.44 \\
7.3\end{array}$ & $\begin{array}{l}0.56 \\
7.6\end{array}$ & $\begin{array}{l}0.50 \\
6.5\end{array}$ & $\begin{array}{l}0.38 \\
7.3\end{array}$ \\
\hline phosphate & & & & & & \\
\hline Total, mg/1 P & 0.025 & 0.035 & 0.028 & 0.032 & 0.03 & 0.03 \\
\hline ortho, mg/1 P & 0.01 & 0.01 & 0.01 & 0.01 & 0.01 & 0.01 \\
\hline Solids & & & & & & \\
\hline Dissolued, mg/1 & 787.8 & 900 & 100 & 7600 & 100 & 100 \\
\hline Suspended, mg/1 & 14 & 4 & 5 & 4 & 6 & 8 \\
\hline Sulfate, mg/ $\mathrm{SO}_{4}$ & 10 & 12 & $1 \mathrm{~A}$ & 12 & 15 & 10 \\
\hline Temperature, ${ }^{\circ} \mathrm{C}$ & $14^{\circ} \mathrm{C}$ & $19^{\circ} \mathrm{C}$ & $22^{\circ} \mathrm{C}$ & $23^{\circ} \mathrm{C}$ & $19^{\circ} \mathrm{C}$ & $13^{\circ} \mathrm{C}$ \\
\hline Turbiaity, NTU & 12 & 1.5 & 2.9 & 2.45 & 2.5 & 1.7 \\
\hline 21nc, $\mathrm{mg} / 1 \mathrm{zn}$ & 0.025 & 0.03 & 0.01 & 0.015 & 0.02 & 0.02 \\
\hline
\end{tabular}


Tabl ' 14 (Cont'd)

WATRR QUALITY MONTTORING DATA

Forrestal Campus, Princeton Univẹrsity

Station: P1

Monitoring Station Location; Schalk station Creek, 0.75 miles, S.E.

\section{Parameter}

Alkalinity, mg/1 $\mathrm{CaCO}_{3} \mathrm{eq}$

Calciuh Hardnese, mg/i $\mathrm{CaCO}_{3} \mathrm{eq}$.

Chromium

Tatal, mg/1 $\mathrm{Cr}$

Hexavalent, mg/1 $\mathrm{Cr}$

Copper, $\mathrm{mg} / \mathrm{t}$ ou

Iron, mg $/ 1$ Fe

Ni trogen

Nitrate, $\mathrm{mg} / 1 \mathrm{~N}$

oil/Grease, ng/1

$\mathrm{ph}$

Phosphate

$$
\text { Total, mg/t p }
$$

or tho, mg/1 $\mathrm{P}$

Solids

Dissolved, mg/1

Suspended, $\mathrm{mg} / 1$

Sulfate, $\mathrm{mg} / 1 \mathrm{SO}_{4}$

Temperature, ${ }^{\circ} \mathrm{C}$

Turbidity, Nru

zinc, mg/1 in $\begin{array}{lll}10 / 16 / 84 & 11 / 20 / 84 & 12 / 31 / 84 \\ 10: 45 \text { a.m. } & 10: 15 \text { A.M. } & 11: 45 \text { a.m. }\end{array}$

10

24

0.05

0.01

0.05

0.33

0.60

0.32

6.6

0.04

0.01

129

10

11

$13^{\circ} \mathrm{C}$

1.4

0.02

\section{1}

22

0.05

0.01

0.04

0.30

0.54

0.34

7.1

0.03

0.01

125

7

10

1.5

0.02
14

32

$<0.1$

$<0.1$

0.009

1.0

1.7

$<1$

7.0

0.02

0.01

54

9

12

$3^{\circ} \mathrm{C}$

3. 1

0.03 
Table 15

WATER QUALITY MONITORING DATA

Forrestal Campus, Princeton University

Station: $\mathrm{F} 2$

Monitoring station Location: George Davison Rd, Creek, 2.6 miles, S.E.

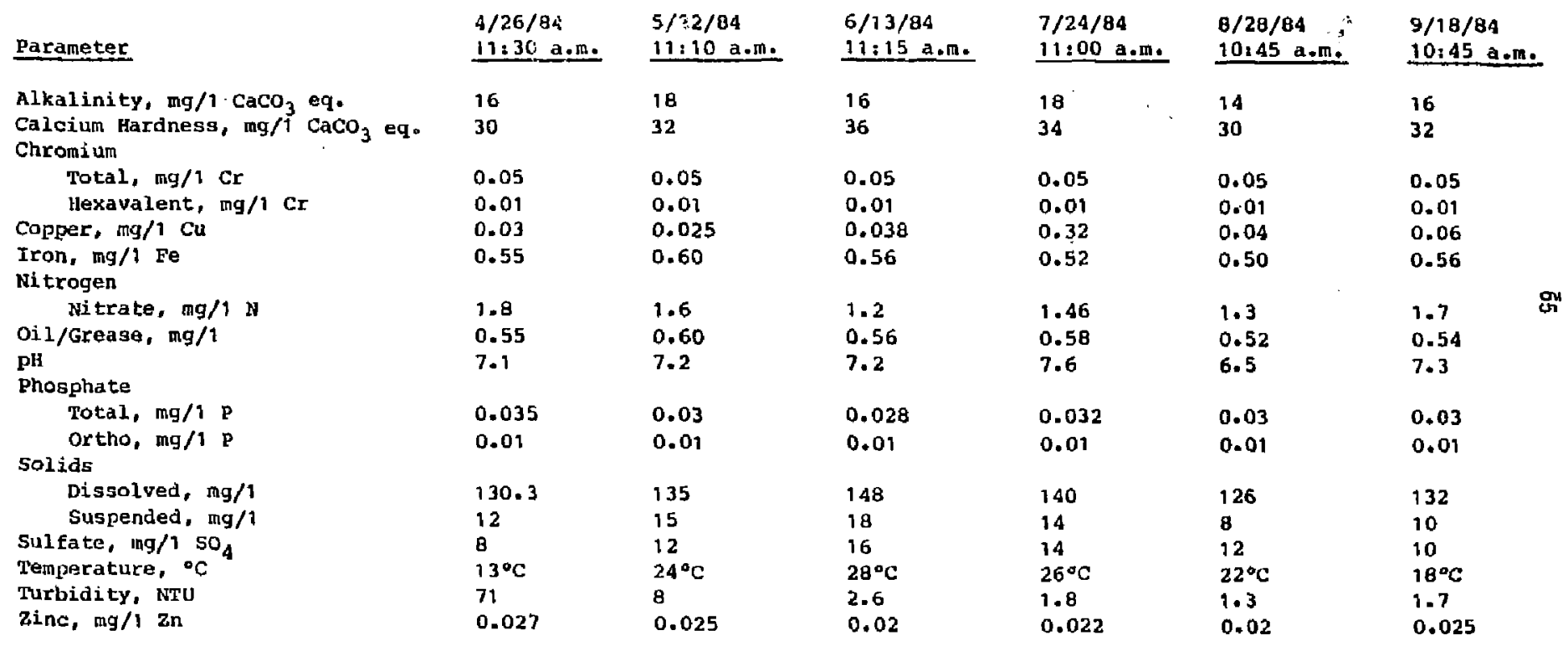


Table 15 (Cont'd)

WATER QUALITY MONITORING DATA

Forrestal Campus. Princeton University

$$
\text { Station: P2 }
$$

Monizoring Station Location: George Davioon Rd, Creek, 2.6 miles, S.E.

Parameter

Alkalinity, mg/1 $\mathrm{CaCO}_{3} \mathrm{eq}$.

Calcium Hardness, $\mathrm{mg} / 1 \mathrm{CaCO}_{3} \mathrm{eq}$. Chromium

$$
\text { Total, } \mathrm{mg} / \mathrm{l} \mathrm{Cr}
$$

Hexavalent, $\mathrm{gg} / 1 \mathrm{Cr}$ Copper, mg/ $\mathrm{Cu}$

Iron, $\mathrm{mg} / 1 \mathrm{Fe}$

Ni trogen

Nitrate, mg/l N

Oil/Grease, mg/1

pH

Phosphate

Total, mg/1 P

ortho, $\mathrm{mg} / 1 \mathrm{P}$

Solids

Dissolved, mg/ 1

Suspended, mg/1

Sulfate, mg/ $1 \mathrm{SO}_{4}$

Temperature, ${ }^{\circ} \mathrm{C}$

Thirbidity, NTU

zine, $\mathrm{mg} / 1 \mathrm{zn}$ \begin{tabular}{lll}
$10 / 16 / 84$ & $11 / 20 / 84$ & $12 / 31 / 84$ \\
$10: 45$ a.m. & $10: 30$ a.m. & $11: 55$ a.m. \\
\hline
\end{tabular}

36

20

34

10

44

$0.05 \quad 0.05$

0.01

0.07

0.60

0.01

0.08

0.62

$<.01$

.006

.82

1.6

0.52

1.4

$2 \cdot 3$

$<1$

$7.1 \quad 6.8$

0.06

7.1

0.03

0.04

0.01

0.01

$\begin{array}{lll}184 & 178 & 100 \\ 12 & 6 & 3\end{array}$

$11-10 \quad 28$

$16^{\circ} \mathrm{C} \quad 5^{\circ} \mathrm{C} \quad 7^{\circ} \mathrm{C}$

$0.9 \quad 1.0 \quad 3.5$

$\begin{array}{lll}0.02 & 0.02 & 0.02\end{array}$ 
Table 16

WATIR QUALITY MONITORING DATA

Forrestal Campus, Princeton Uni yersity

station: $M 1$

Monitoring Station Location: Miligtone River, 1.8 miles

\section{Parameter}

Alkalinity, mg/1 $\mathrm{CaCO}_{3}$ eq.

Calcium Hardness, mg/1 $\mathrm{CaCO}_{3} \mathrm{eq}$. Chromium

\section{Total, mg/l $\mathrm{Cr}$}

Hexavalent, mg/ $\mathrm{Cr}$

Copper, $m g / 1 \mathrm{cu}$

Iron, $\mathrm{mg} / 1 \mathrm{Fe}$

Ni trogen

$$
\text { Nitrate, } m g / 1 \mathrm{w}
$$

Oil/Greage, mg/l

pl1

Phosphate

Total, mg/l p Ortho, $\mathrm{mg} / 1 \mathrm{P}$

Solids

Dissolved, mg/1

suspended, mg/

Sulface, $\mathrm{mg} / 1 \mathrm{SO}_{4}$

Temper a ture, ${ }^{\circ} \mathrm{C}$

Turbidity, NIU

Zine, ing/ $1 \mathrm{zn}$

\section{$4 / 26 / 84$ \\ $11: 45$ a.m. $11: 25$ a.m.}

22

36

0.05

0.01

0.06

0.32

1.2

0.35

7.0

0.02

0.01

1.2.). 3

8

12

$13^{\circ} \mathrm{C}$

23

0.022

3.2

0.028
$6 / 13 / 84$

11:35 a.m.

20

32

0.05

0.01

0.07

0.04

1.7

0.54

7.5

0.028

0.018

158

14

12

$28^{\circ} \mathrm{C}$

4.2

0.022
$7 / 24 / 84$

11:30 a.m

\section{2}

36

0.05

0.01

0.06

0.034

1.35

0.45

7.5

0.025

0.01

145

12

12

$25^{\circ} \mathrm{C}$
5

0.024
$8 / 28 / 84$

11:00 a.m.

\section{6}

40

0.05

0.01

0.07

0.038

1.60

0.50

7.0

0.03

0.01

161

16

14

$23^{\circ} \mathrm{C}$

1.7

0.026
9/18/84

11:15 a.m.

24

36

0.05

0.01

0.05

0.26

1. 3

0.38

7.4

0.025

0.01

140

io

12

$17^{\circ} \mathrm{C}$

1.8

0.02 
Table 16 (Cont'd)

WATER QUALITY MONITORING DATA

Forrestal Campus, Princeton University

Station: $M l$

Monitoring Station Location: Millstone River, 1.8 miles

\section{Parameter}

Alkalinity, $\mathrm{mg} / 1 \mathrm{CaCO}_{3}$ eq.

Calcium Hardnesa, $\mathrm{mg} / 1 \mathrm{CaCO}_{3} \mathrm{eq}$.

Chromium

Total, mg/1 Cr

Hexavalent, $\mathrm{mg} / 1 \mathrm{Cr}$

Copper, mg/i $\mathrm{Cu}$

Iron, mg/l Fe

Nitrogen

Nitrate, mg/l $\mathrm{N}$

oil/Grease, mg/1

$\mathrm{pH}$

Phosphate

Total, $\mathrm{mg} / \mathrm{h}$ Ortho, $\mathrm{mg} / 1 \mathrm{p}$

Solids

nissolved, ng/i

Suspended, mg/1

sulfate, $\mathrm{mg} / 1 \mathrm{SO}_{4}$

Temperature, ${ }^{\circ} \mathrm{C}$

Turbidity, NTU

zinc, myil $\mathrm{zn}$
$10 / 16 / 84 \quad 11 / 20 / 84 \quad 12 / 31 / 84$

$11: 15 \mathrm{a} . \mathrm{m} . \quad 11: 00 \mathrm{a.m.} \quad 12: 15 \mathrm{p.m}$.

28

34

25

30

24

48

$0.05 \quad 0.05<.01$

$0.010 .09<.01$

$0.06 \quad 0.07 \quad 0.01$

0.200 .26 . 0.97

$\begin{array}{lll}1.4 & 1.5 & 2.4 \\ 0.32 & 0.30 & 1\end{array}$

$0.320 .30 \quad 1$

$\begin{array}{lll}6.9 & 7.1 & 6.4\end{array}$

$0.05 \quad 0.04 \quad 0.12$

$0.02 \quad 0.02 \quad 0.08$

$\begin{array}{lll}194 & 175 & 100\end{array}$

$12 \quad 10 \quad 7$

$12 \quad 12 \quad 22$

$15^{\circ} \mathrm{C} \quad 6^{\circ} \mathrm{C} \quad 5^{\circ} \mathrm{C}$

$1.1 \quad 2.4 \quad 4.1$

$\begin{array}{lll}0.02 & 0.02 & 0.03\end{array}$ 


\section{Table 17}

WATER OUALITY MONITORING DATA

Forrestal Campus, Princeton Univergity

$$
\text { Station: H4 }
$$

Monitoring Station Location: Well No. 4 at $C$ Site (Non-operational)

\section{Parameter}

Alkalinity, mg/1 $\mathrm{CaCO}_{3} \mathrm{eq}$.

Calcium llardness, mc/1 $\mathrm{CaCO}_{3} \mathrm{eq}$.

Copper, mg/1 Cu

Fecal coliform Count, MPN/100 $\mathrm{ml}$.

Iron, $m g / 1 \mathrm{Fe}$

Nitrogen

Total mg/1 $\mathrm{N}$

pH

Nitrate, $\mathrm{mg} / \mathrm{d} \mathrm{No}_{3}$

Phosphate

Total, mg/1 P

ortho, $\mathrm{mg} / 1 \mathrm{p}$

Manganese, mg/l Mn

Chloride, $\mathrm{mg} / \mathrm{l} \mathrm{cl}$

Solids

Dissolved, $\mathrm{mg} / 1$

sulfate, $m g / 1 \mathrm{SO}_{4}$

Teлperature, ${ }^{\circ} \mathrm{C}$

Turbidity, NTU

\section{$6 / 13 / 84-9 / 19 / 84$}

9:00 a.m. 10:30 a.m.

$\begin{array}{ll}8 & 10 \\ 72 & 70 \\ 0.05 & 0.04 \\ 0 & 0 \\ 0.95 & 0.86 \\ & \\ 0.6 & 0.75 \\ 2.64 & 3.3 \\ 7.1 & 7.0\end{array}$


Table 18

WATER QUALITY MONITORING DATA

Forregtal Campus, Princeton Univeraity

$$
\text { Station: w5 }
$$

Monitoring Station Location: Well No. 5 at 6 Site (Non-operational)

\begin{tabular}{|c|c|c|}
\hline & $6 / 13 / 84$ & $9 / 18 / 84$ \\
\hline Parameter & $9: 30 \mathrm{a} \cdot \mathrm{m}$. & $10: 45$ a.m. \\
\hline Alkalinity, $\mathrm{mg} / 1 \mathrm{CaCO}_{3} \mathrm{eq}$. & 42 & $\overline{42}$ \\
\hline Calcium Hardness, mg// $\mathrm{CaCO}_{3} \mathrm{eq}$. & 34 & 32 \\
\hline Copper, mg/1 cu & 0.07 & 0.06 \\
\hline Fecal Coliform count, MPN/100 ml. & 0 & 0 \\
\hline $\begin{array}{l}\text { Iron, mg/1 Fe } \\
\text { Nitrogen }\end{array}$ & 0.6 & 0.65 \\
\hline Total mg/1 N & 1.2 & 1.3 \\
\hline Nitrate, $\mathrm{ng} / 1 \mathrm{NO}_{3}$ & 5.28 & 5.73 \\
\hline pH & 6.8 & 6.7 \\
\hline \multicolumn{3}{|l|}{ Phosphate } \\
\hline Total, mg/l $\mathrm{P}$ & 0 & $\mathbf{0}$ \\
\hline Ortho, $\mathrm{mg} / 1 \mathrm{P}$ & 0 & 0 \\
\hline Manganese, mg/1 Mn & 0.1 & 0.12 \\
\hline Chloride, $\mathrm{mg} / \mathrm{l} \mathrm{cl}$ & 8 & B \\
\hline \multicolumn{3}{|l|}{ Solids } \\
\hline Dissolved, mg/1 & 135 & 138 \\
\hline Sulfate, ing/1 $\mathrm{SO}_{4}$ & 16 & 16 \\
\hline Temperature, ${ }^{\circ} \mathrm{C}$ & $16^{\circ} \mathrm{C}$ & $12^{\circ} \mathrm{C}$ \\
\hline Turbiaity, NTU & 5.5 & 3.5 \\
\hline
\end{tabular}


TABLE 19

FERTILIZER, PESTICIDES AND KERBICIOES APPLICATION (C185)

\section{FERT ILI ZER}

A Site

B Site

C Site

D Site

PESTICIDES
A Site
B S1 te
C site
D Site

(Applied by Univergity)

$20-12-8504$ organic

$$
\begin{array}{r}
10001 \mathrm{bs} . \\
12001 \mathrm{bs} . \\
1000 \text { 16s. } \\
800 \text { 1bs. }
\end{array}
$$

(Applied by Unfyersity)

No perticides applied

Sevin - 600 gallons for Japanese beetle control

No pesticides applied

No pesticldes applied

\section{SERBICIDES}

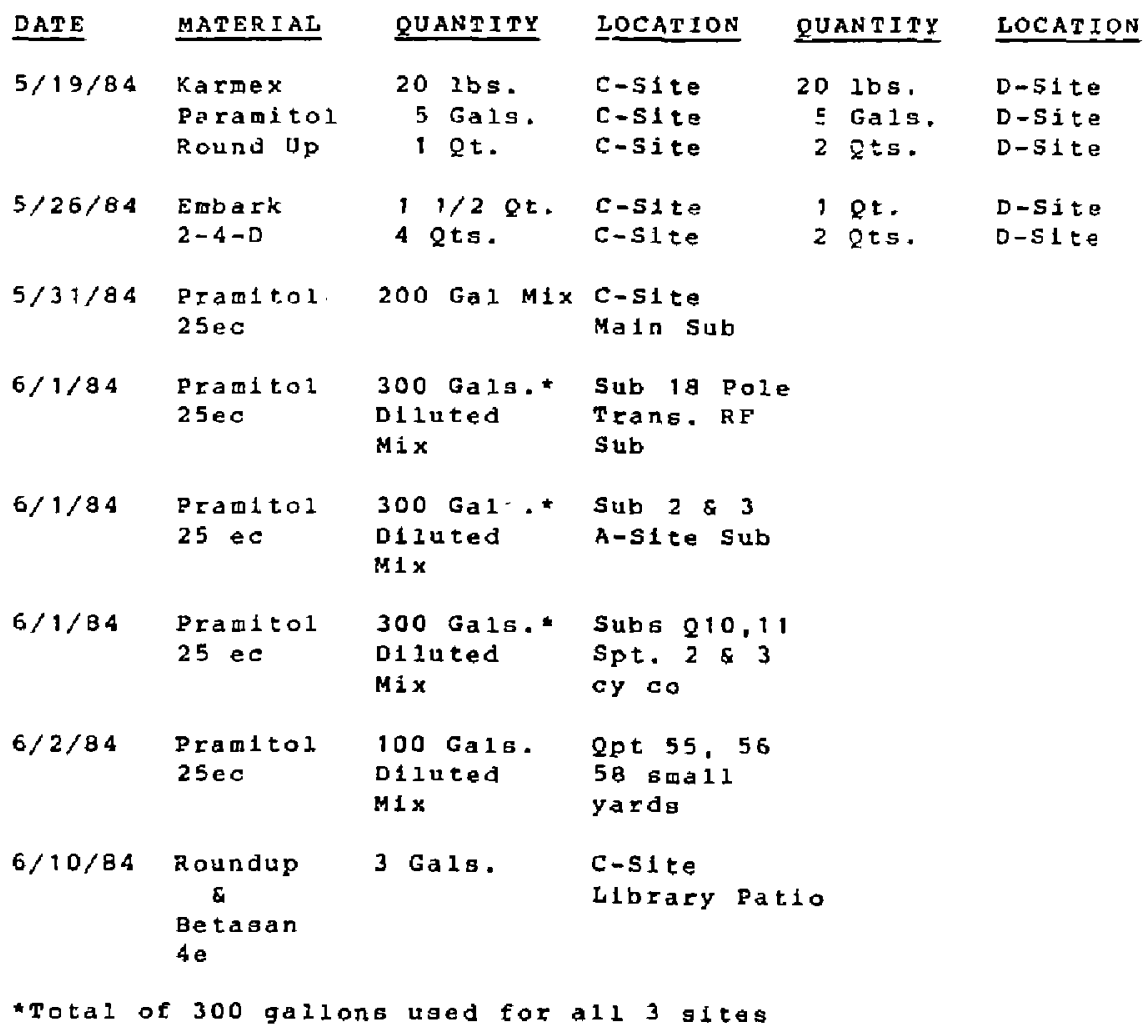


Tab2e 19 (Cont'd.)

\begin{tabular}{|c|c|c|c|c|c|}
\hline DATE & MATERIAL & QUANTITY & LOCATION & QUANTITY & LOCATION \\
\hline $6 / 18 / 84$ & $\begin{array}{l}\text { Pramitol } \\
25 \mathrm{ec}\end{array}$ & 35 GaIs. & $\begin{array}{l}\text { Canal Pugp } \\
\text { Houge }\end{array}$ & & \\
\hline $7 / 28 / 84$ & $\begin{array}{l}\text { Erbark } \\
\text { Roundup } \\
\text { Pramitol } \\
2-4-D\end{array}$ & $\begin{array}{l}11 / 2 \text { Qts. } \\
1 \text { Qt. } \\
1 / 2 \text { Gal. } \\
4 \text { Qts. }\end{array}$ & $\begin{array}{l}\text { C-site } \\
\text { C-site } \\
\text { C-site } \\
\text { C-site }\end{array}$ & $\begin{array}{l}1 \mathrm{gt} . \\
2 \mathrm{gals} . \\
2 \mathrm{gts} .\end{array}$ & $\begin{array}{l}0-51 \text { te } \\
D-S 1 t e \\
D-S 1 t e\end{array}$ \\
\hline $8 / 13 / 84$ & $\begin{array}{r}\text { PramitoI } \\
25 e c\end{array}$ & $\begin{array}{l}70 \text { Gals. } \\
\text { Total M } 1 x\end{array}$ & $\begin{array}{l}\text { TFTR NBPC } \\
\text { Sub \& } \\
\text { Switch Yard }\end{array}$ & $\begin{array}{l}30 \text { Galg. } \\
\text { Dilute }\end{array}$ & $\begin{array}{l}\text { B-site } \\
\text { serap } \\
\text { Yard }\end{array}$ \\
\hline $8 / 13 / 84$ & $\begin{array}{l}\text { Pramitol } \\
25 e c\end{array}$ & $\begin{array}{l}10 \text { Gals. } \\
D i 1 \text { uted } \\
M 1 x\end{array}$ & $\begin{array}{l}\text { Oil storage } \\
\text { Tank Area }\end{array}$ & & \\
\hline
\end{tabular}




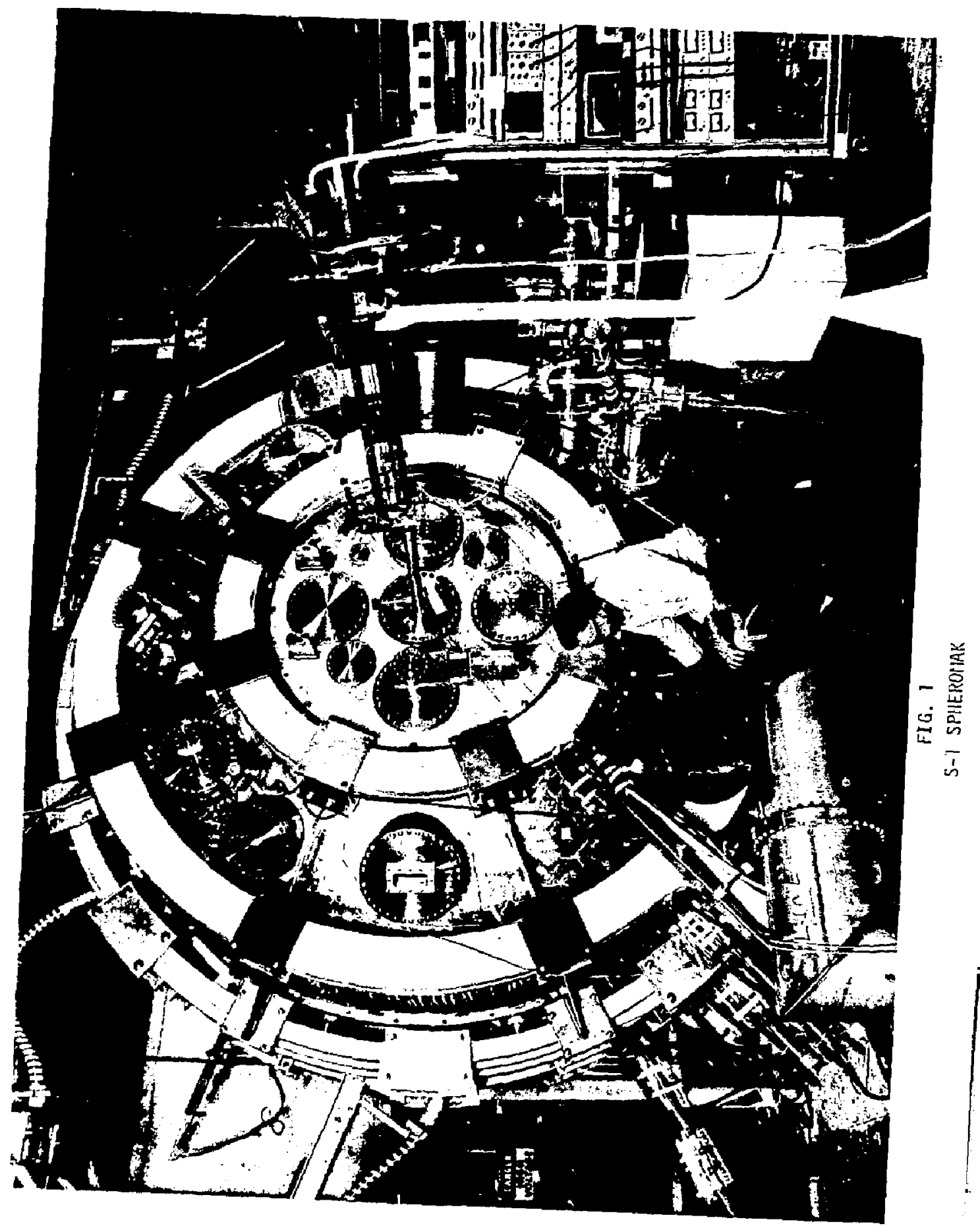




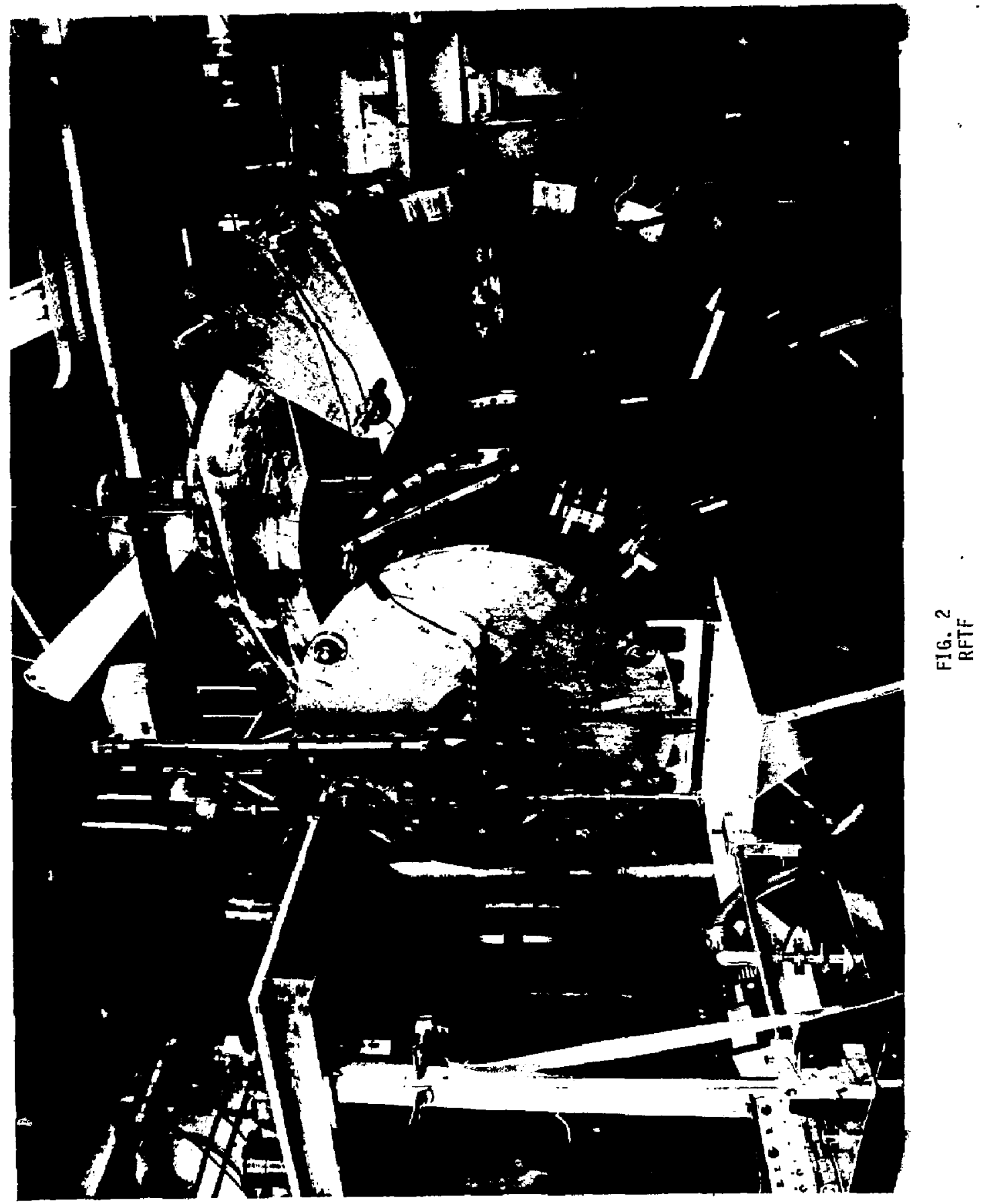




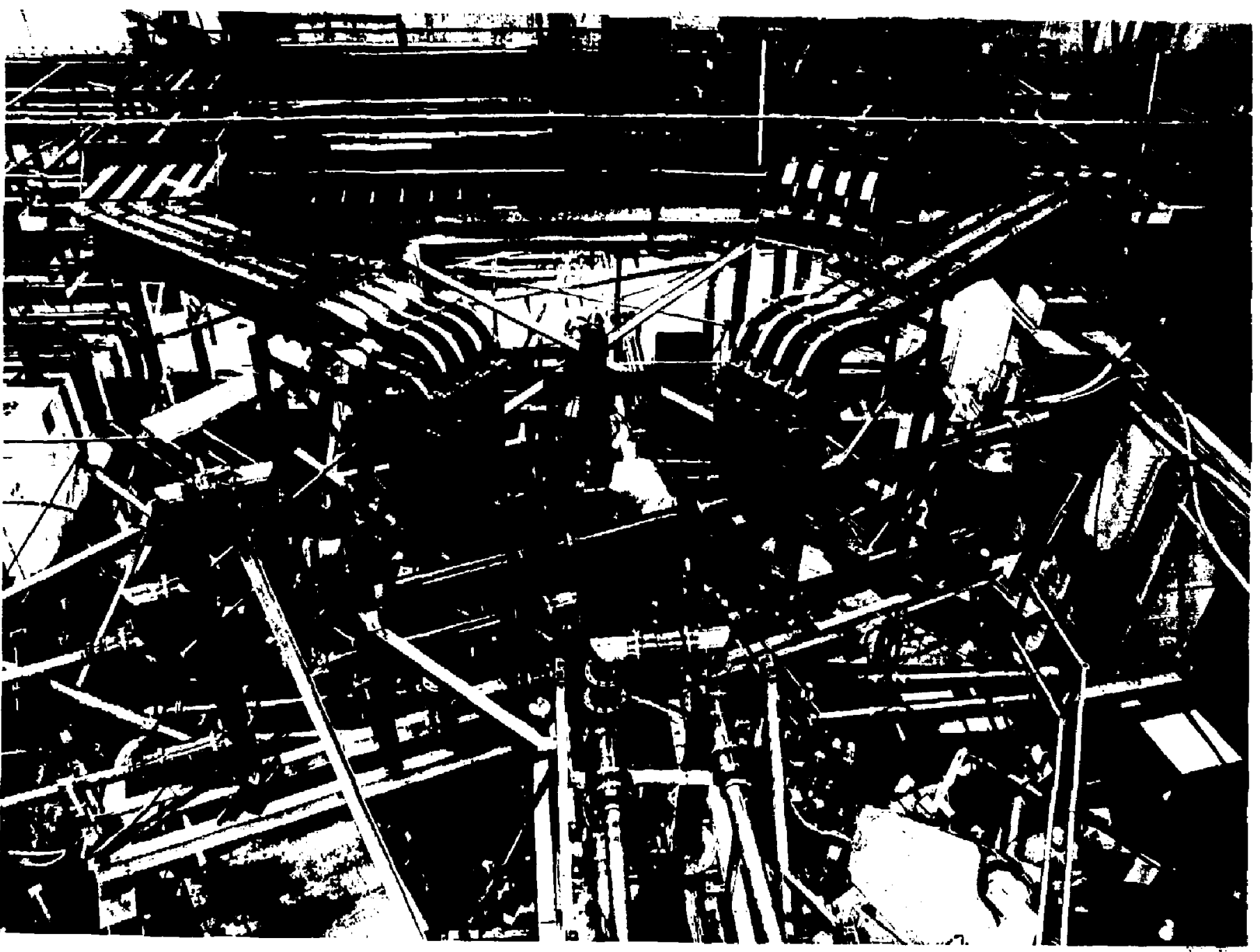

PRINCETON LARGE ${ }^{3}$ TORUS (PLT) 


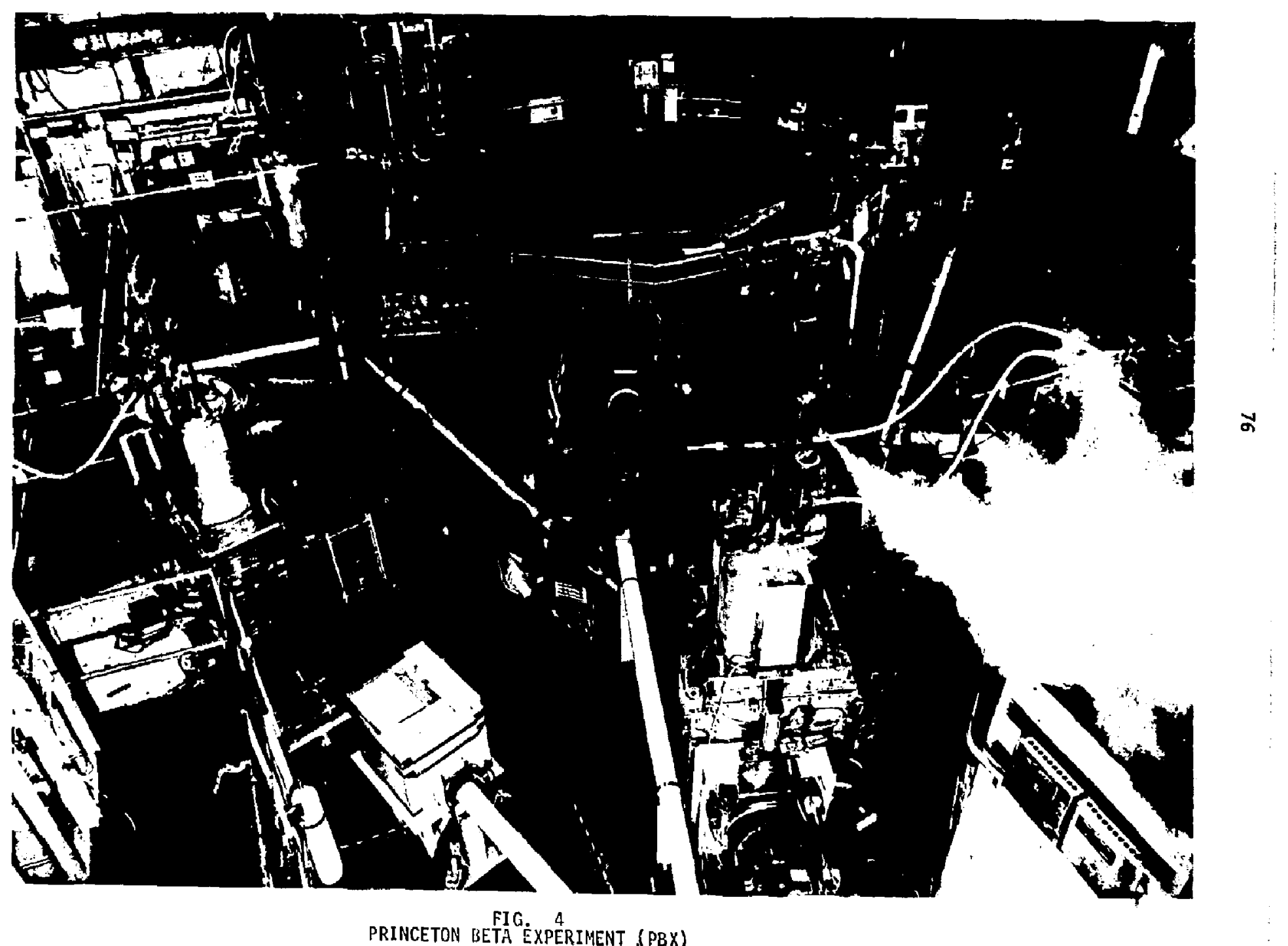




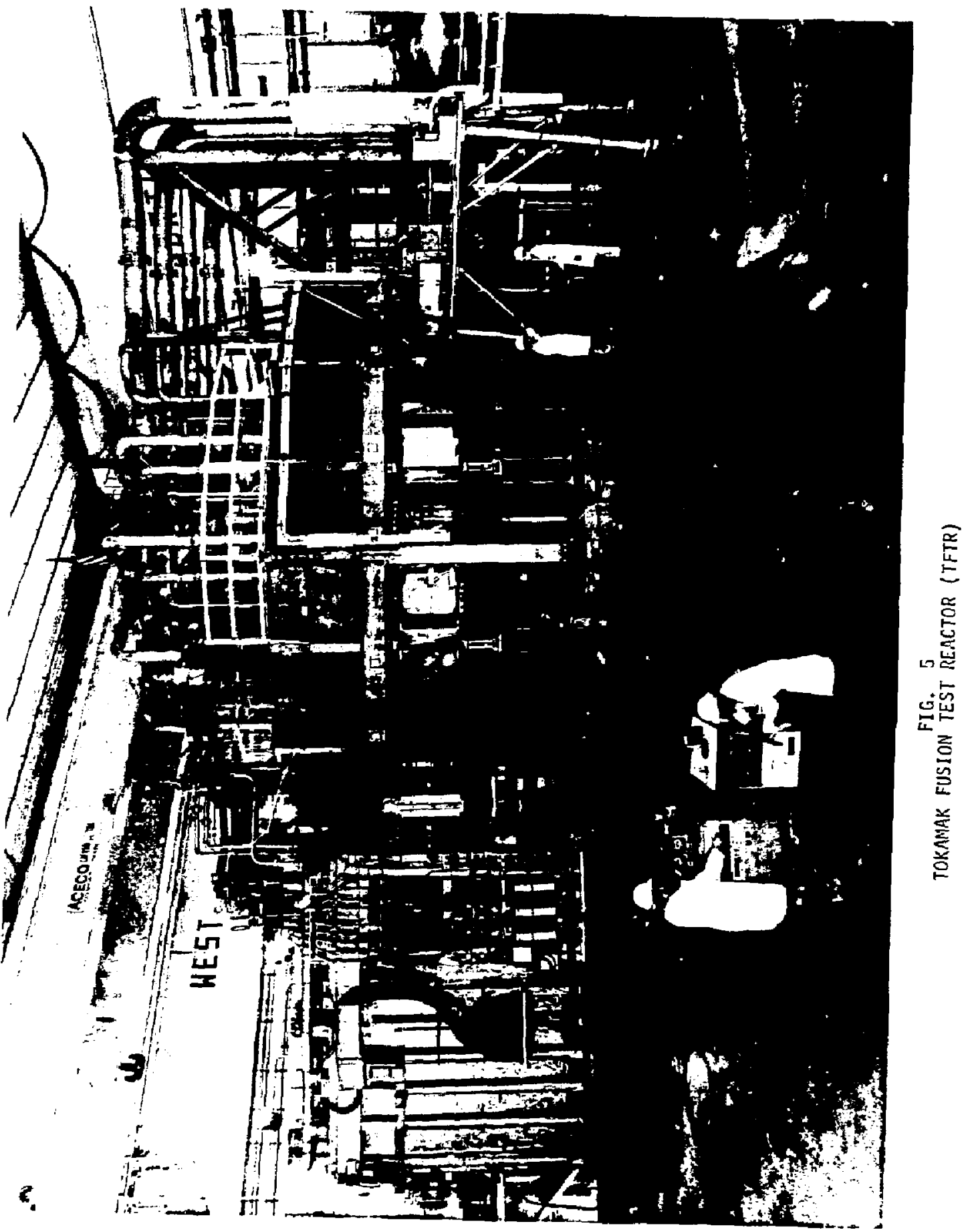




\section{Wind Rose \\ Joint Frequency Data for TFTR B4/ 3 to $85 / 2$ \\ Elevation $60 \mathrm{~m}$ \\ For All Stability Classes ( 100.0\%)}

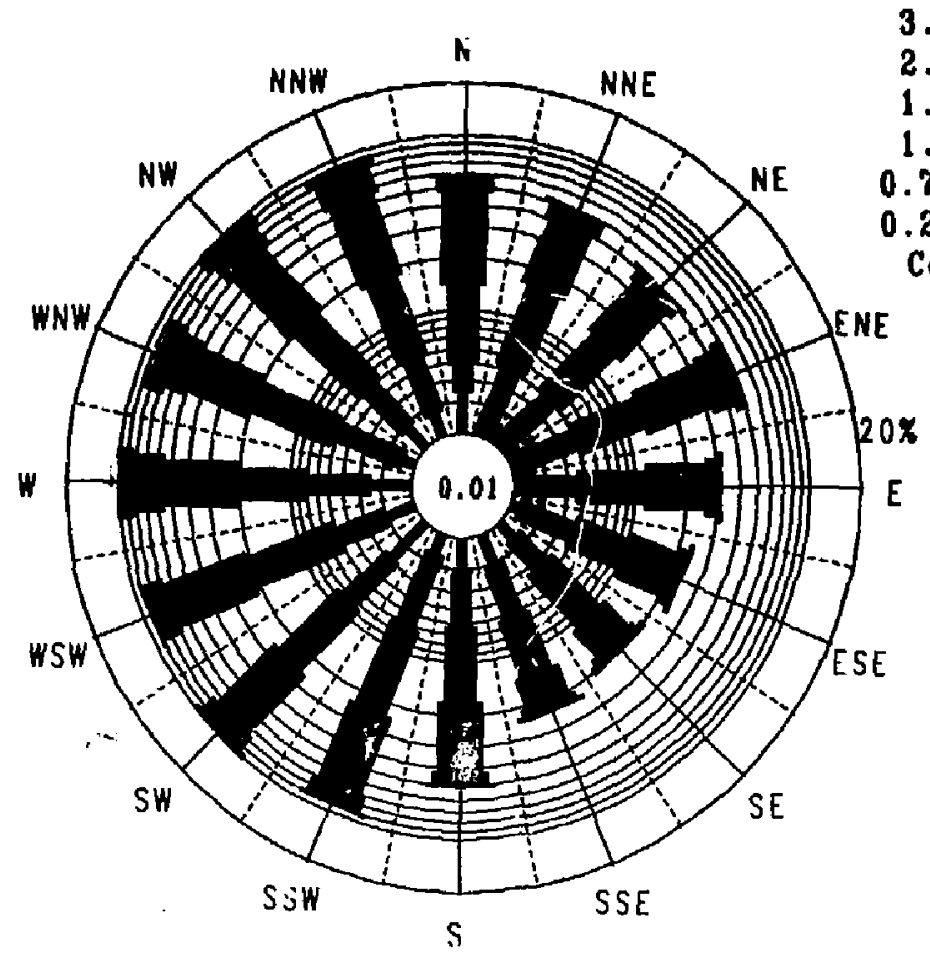

Find Speed ( $\mathbf{n} / \mathbf{s})$

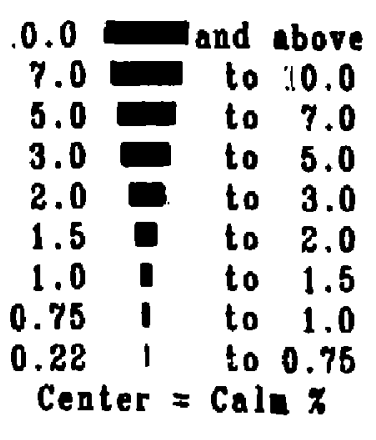

0.0 and above

7.0 to 10.0

5.0 to 7.0

$3.0 \square$ to 5.0

1.0 to 3.0

1.5 to 2.0

0 to 1.5

751 to 1.0

Center $=\mathrm{Caln} \%$ 
FIG. 7

Find Rose

Joint Frequency Data for TFTR $84 / 3$ to $85 / 2$

Elevation $30 \mathrm{~m}$

Por All Stability Classes (100.0\%)

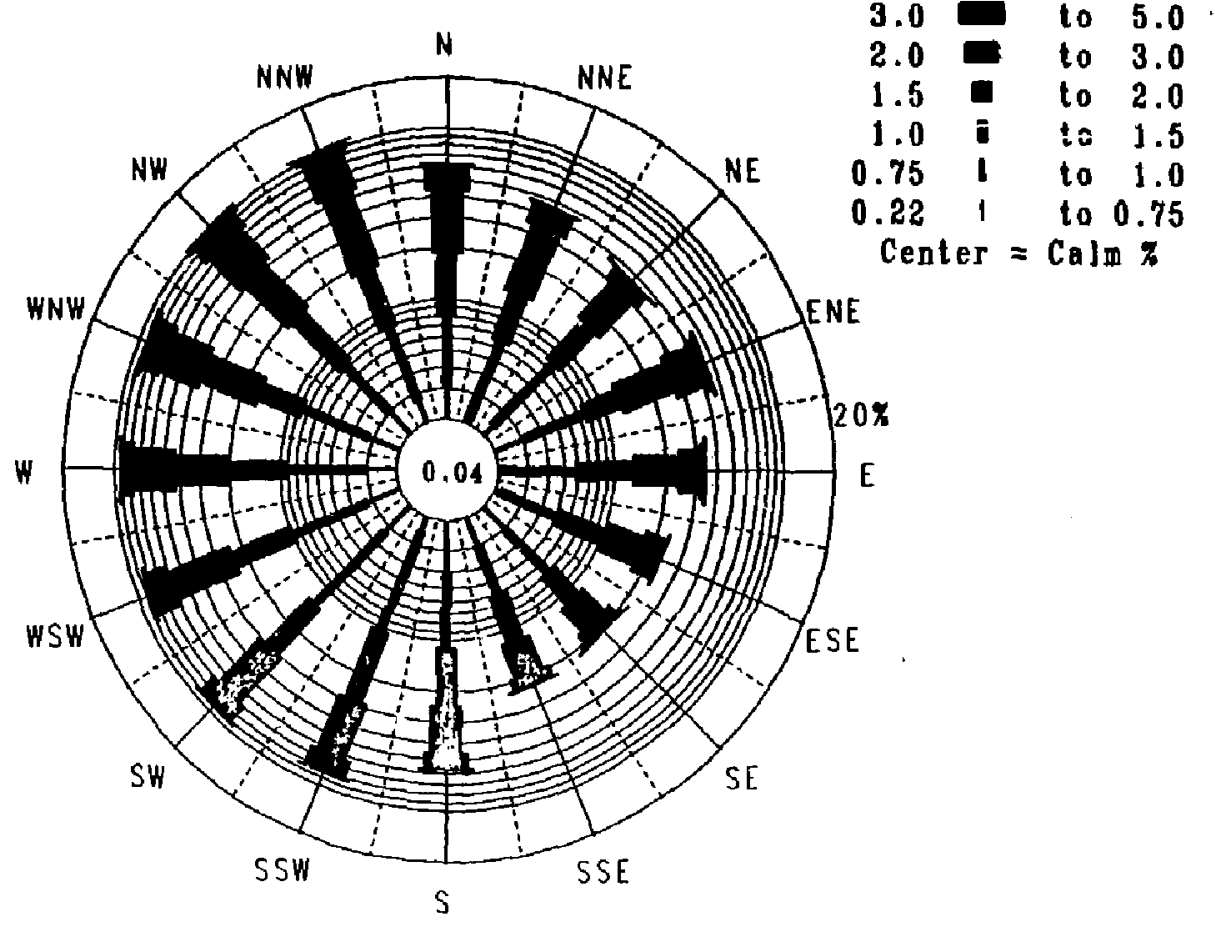




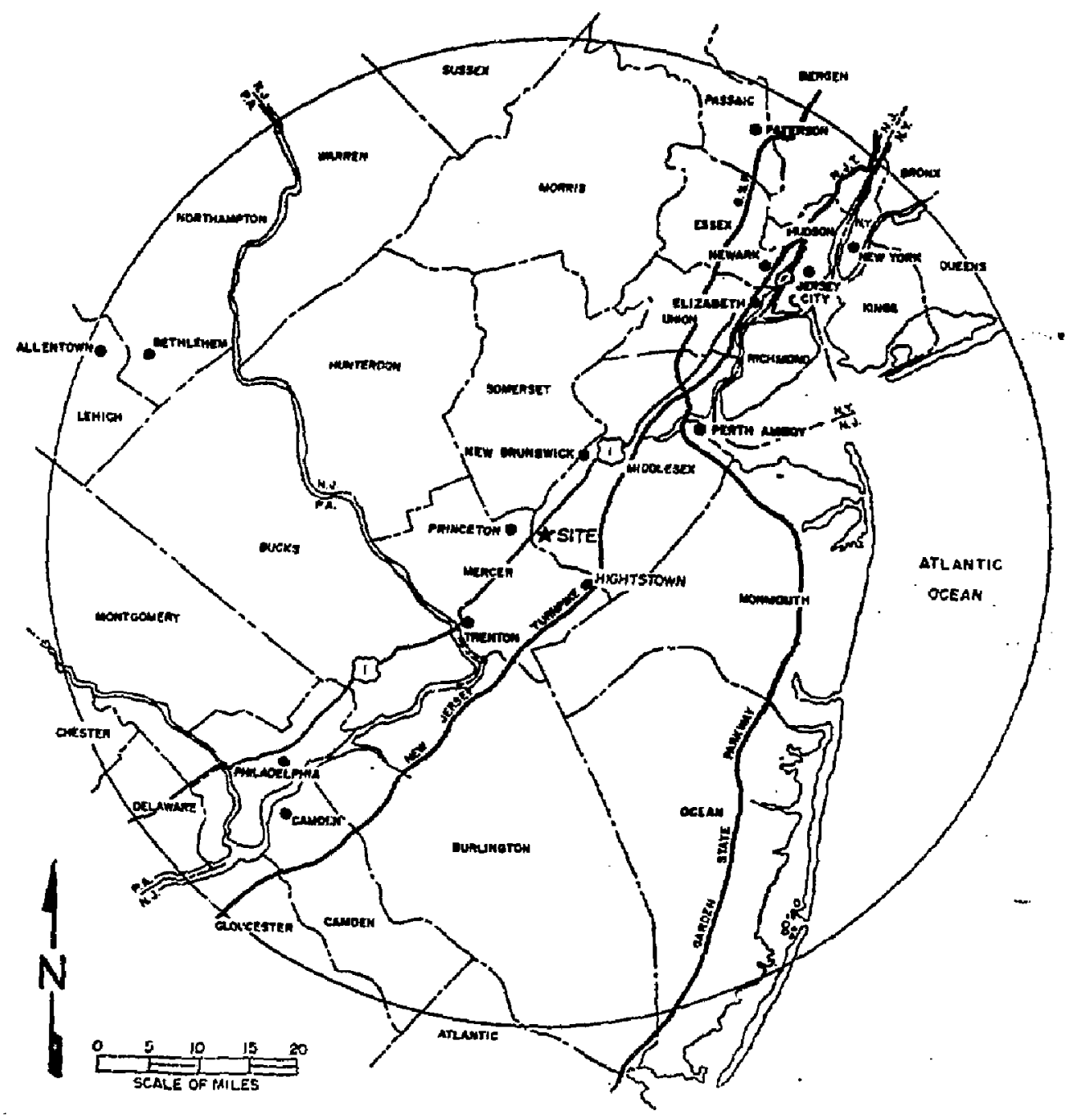

FIG. 8

$30-K_{m}$ (50-MILE) RADIUS OF SITE 


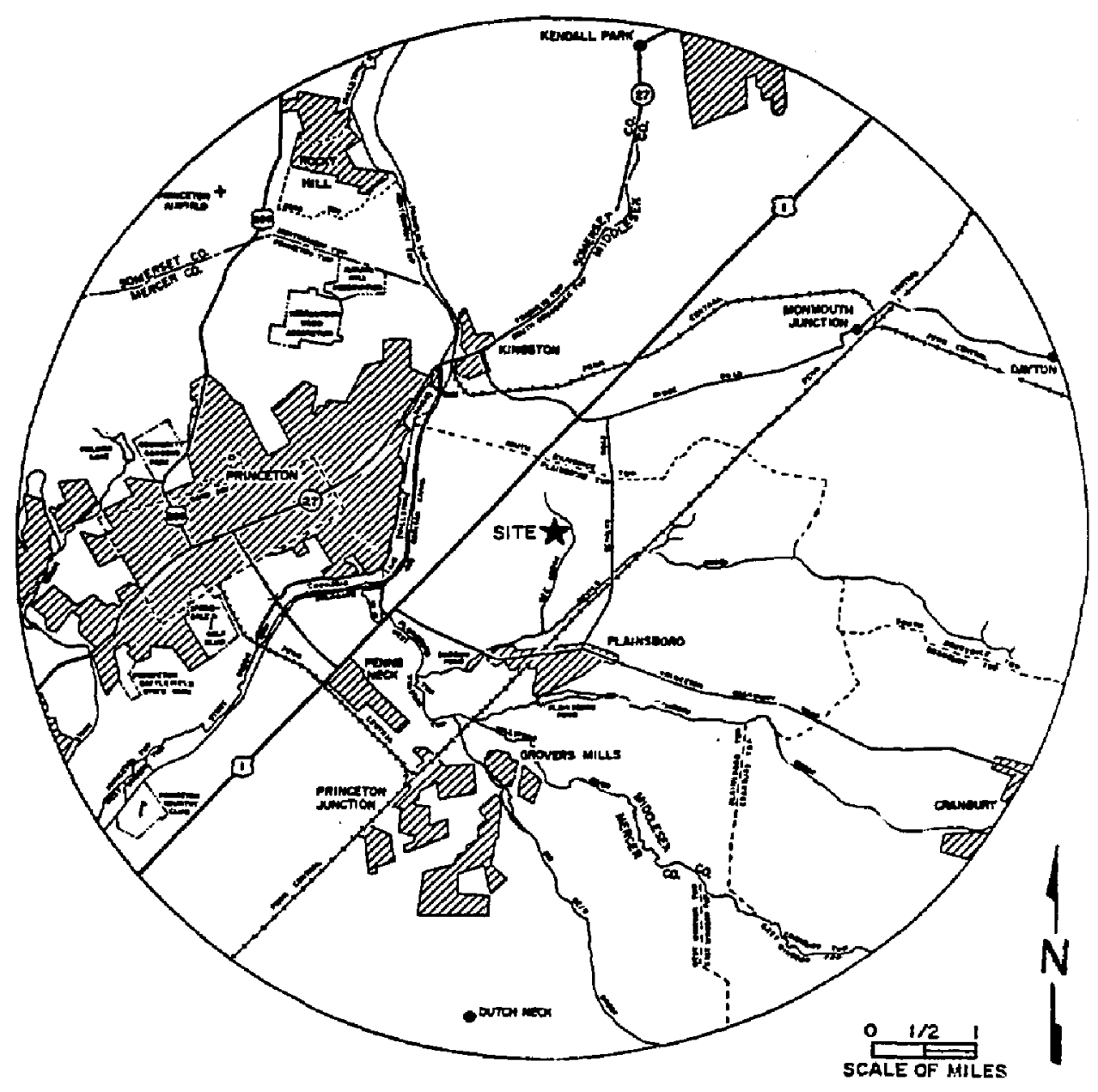

FIG. 9

IMMEDIATE SITE VICINITY

(5-MILE RADIUS) 


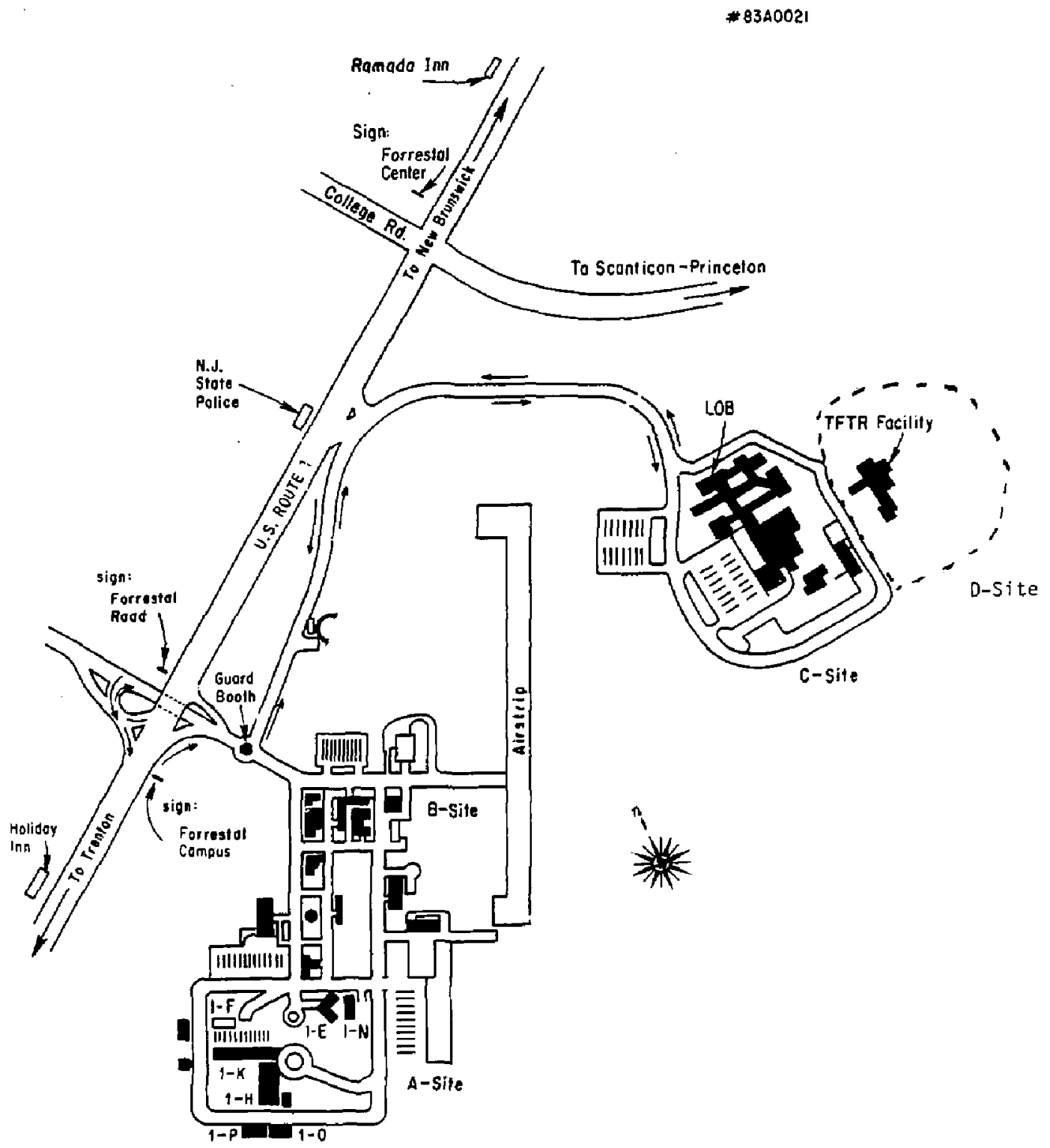

FIG. 10

PLASMA PHYSICS LABORATORY

JAMES FORRESTAL CAMPUS

PRINCETON UNIVERSITY, PRINCETON N.J. 


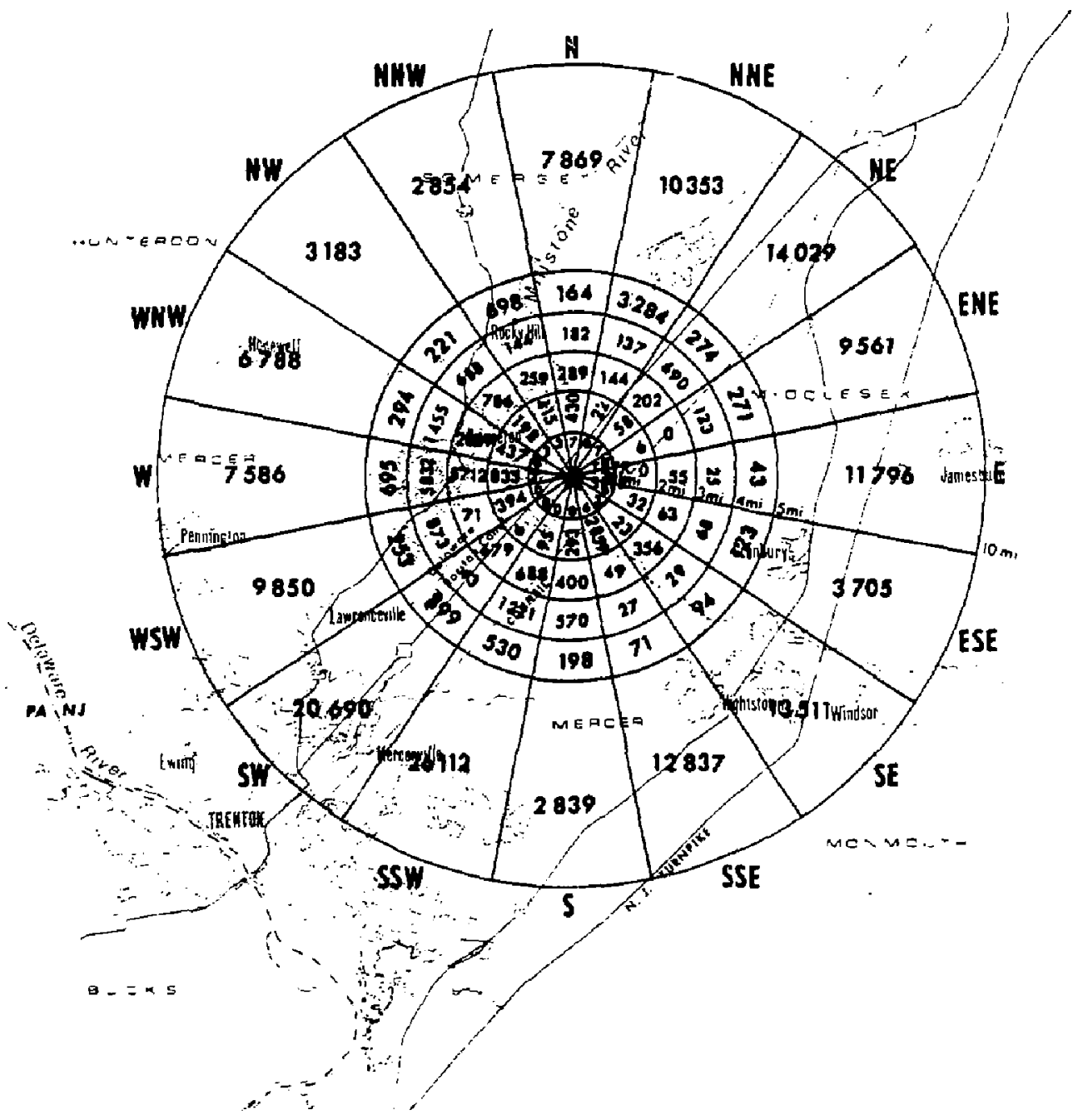

FIG. 11

1974. POPULATION ESTIMATES WITHIN ANNULAR SECTORS 0-10 MILES*

*Taken from TFTR/FSAR

Figures 2.1.2-1 


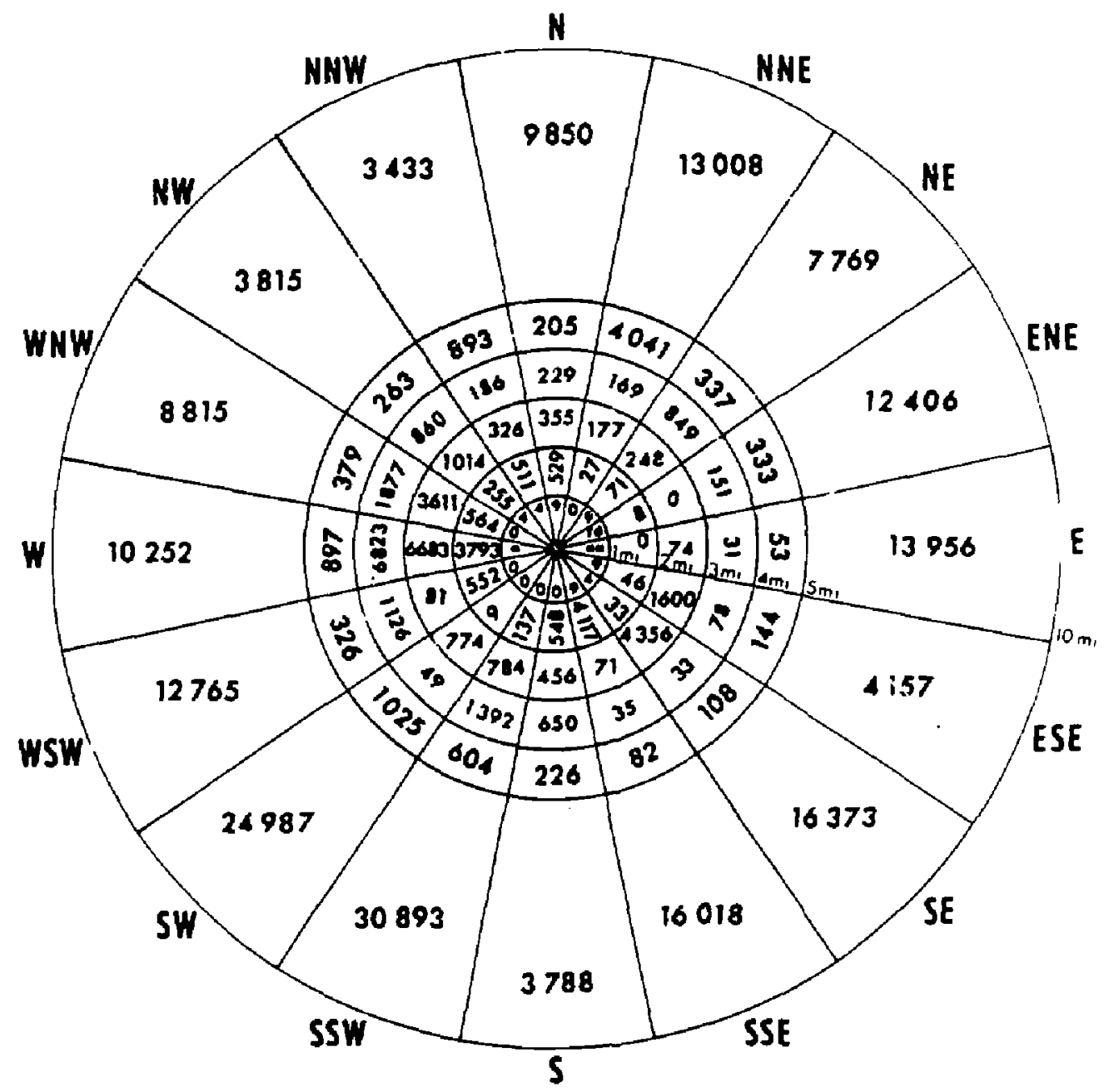

FIG. 12

1985 POPULATION ESTIMATES WITHIN ANNGAL SECTION 0-10 MILES*

*Taken from TFTR/FSAR

F1gure 2.1.2-3 


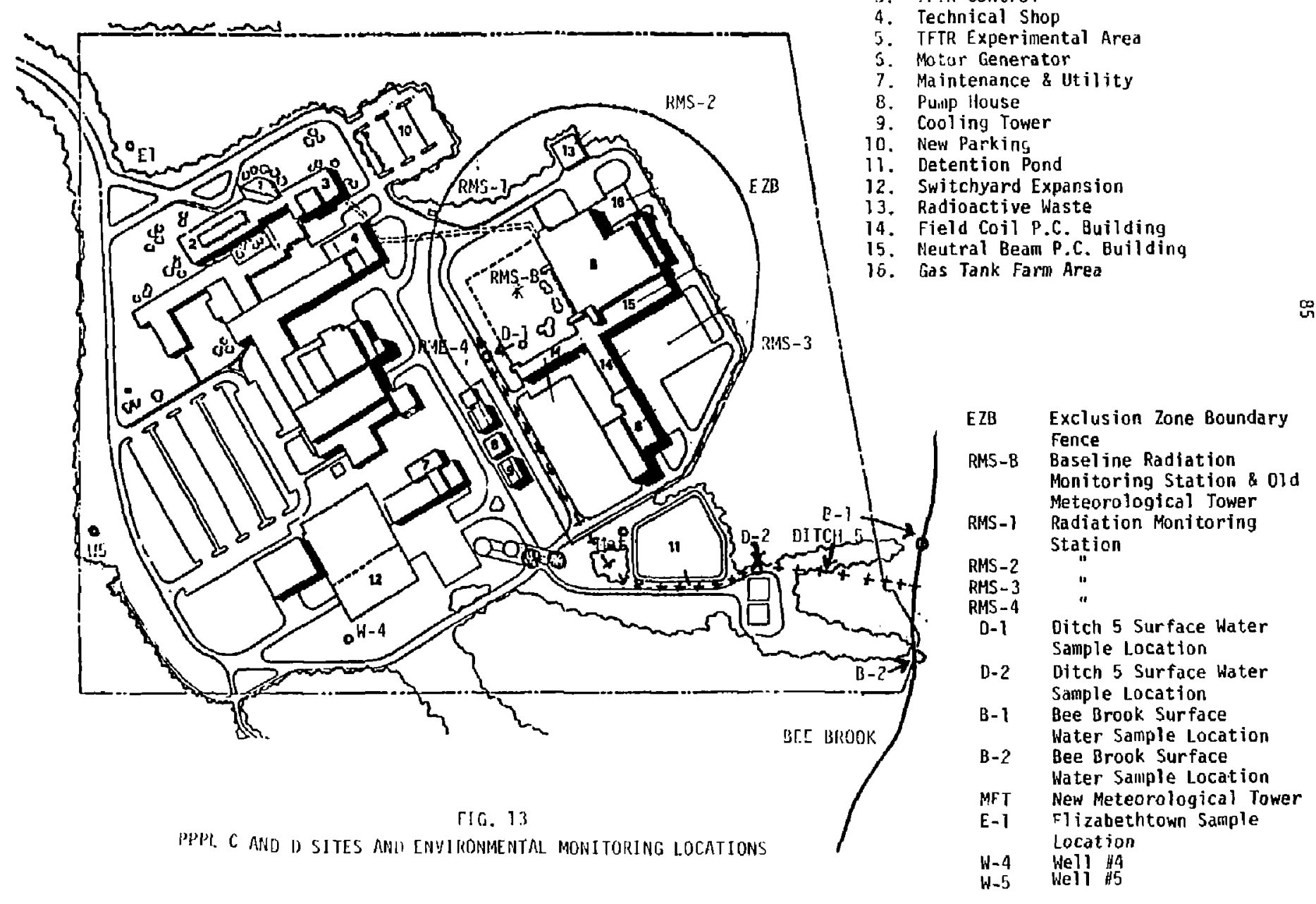




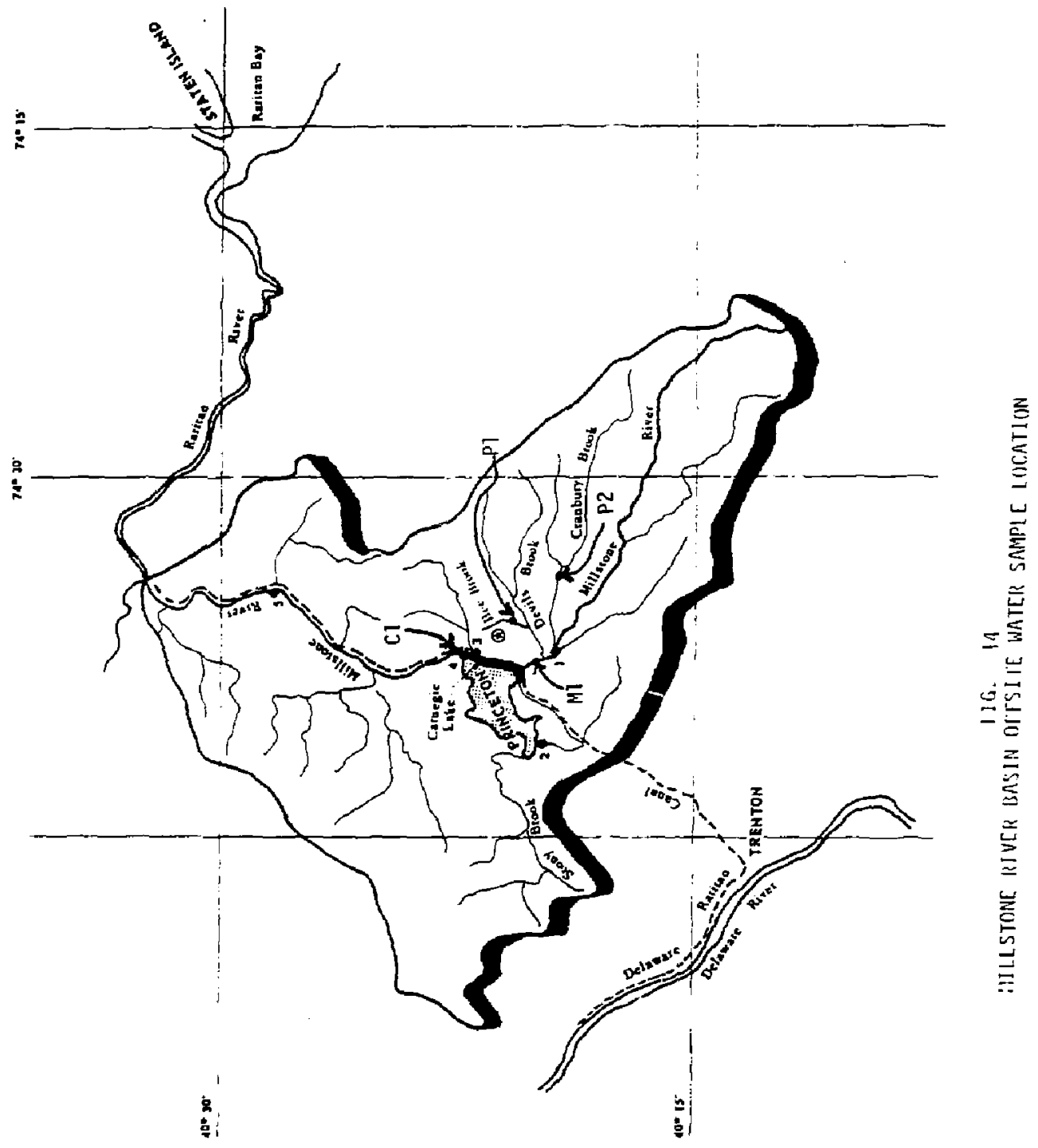

$\rightarrow(\mathrm{z})-1$ 

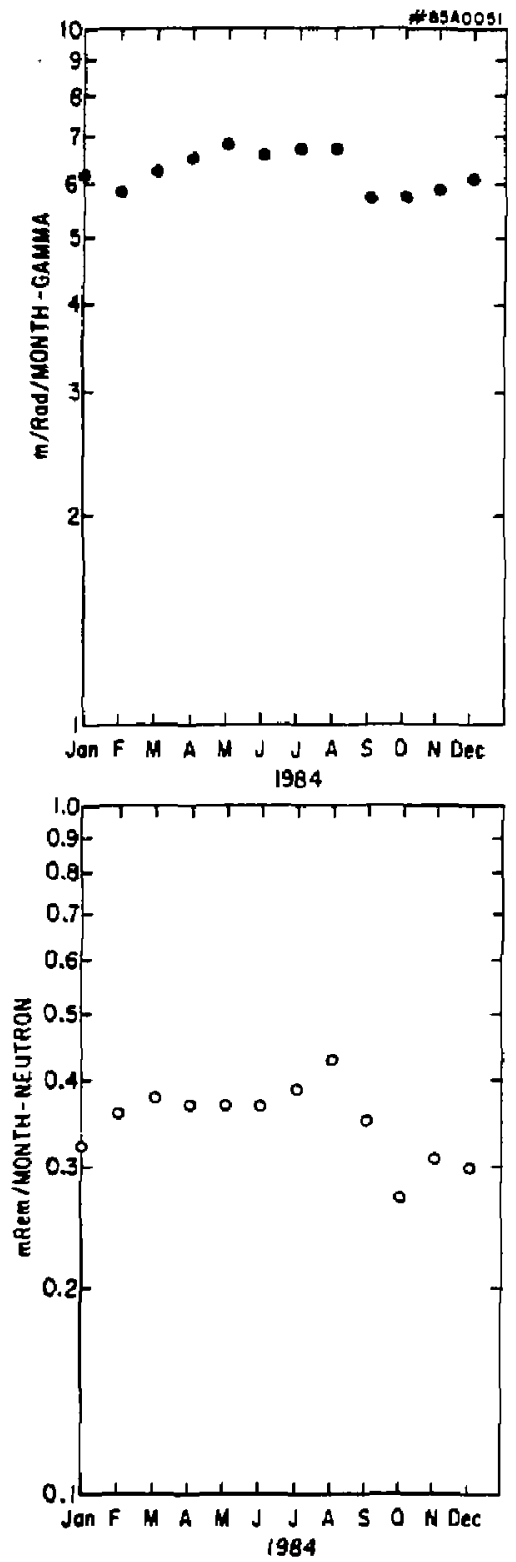

FIG. 15

CYS4 MEUTRON AND GAPIMA ENVI RONHENTAL BACKGROUNO DATA (LOCATION RMS-B) 


\section{EXTERARL DISIRIFUTION _N ADDITLON TO UC-20}

Plactra Res Lab, Austra Nat'l Iniv, Austrasif Dr. Frark J. Paoloni, Thiv of hllongong, AUSTpaLIA Prof. I.R. Jones, Fliniers thiv., AUSTRALTA Prof. M.H. Frenran, miv Sydrey, Australta trof. F. Cap. Inst theo Fhys, Alstruta Prot. Frark ischest, Inst theocetiache, BeIGIN

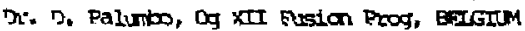
Foole Royale Militaire, Lab de Phrs Plamas, BErGTM Dr. P.H. Sakanaka, thiv pstahual, ENarl. DT. C.R. Janes, Univ of Alberta, CANDA Prof. J. Teictmann, Univ of Hontreal, CANDA Dr. F.M. \$rargard Univ of Saskatohwar, CAvad Prof. S.R. Stenivaran, University of Calgary, CANPADA Prof. Trior $w$. Johnston, INRS-Energie, CANADA Jx. Hannes Batram, oniv setitish oolumbia, Gavad Dr. M.P. Eachinsiki, :Pa Technologies, Ine. CANDOA Chalk River, wacl Lab, Caraph

Dhengon Li, So Inst Fhysics, chnth

ibrary, Tsing tha University, crom Librarian, Instibute of Physics, CHNA Inst Plasma Fhys, Acadenia sinica, GINA ir. Peter Lukac, Kanenskeho Univ, Canorogramkia

The Librarim, collem taboratory, Everavo Prof. Schatoman, observatoire de Nice, ERAMCE J. Reriet, OBH-BF, FRANCE MM Dupas Library, AM Dupas Library, ERANCE Dr. Ion Htal, Acaikny Bibliograptic, fang kaxt pteorint Library, Cent Res Inst PhYs, HUMGaRy r. :Thehan, Parjah Iniversity, RDLa DF. Indra Mohan Lal Das, Fanaras Hind, thiv, INDIA DT. I.K. Chavta, Suth Gijarat Univ, DSIA Dr. 2... Ohtajlani, Vikram univ, InDTA Dr. 3. Dasgupta, Saha Inst, INDIA DT. P. Kew, Fyysical Research Lab, IIDIA T. Phillip Rosenal, Istapl Inst Tech, ISRAEL Prof. S. Oppentan, Tel Aviv Uhiwerstty, ISRAE. Prof.. r. Rostagni, Univ Di Padovr, IIALY Tibrarian, Int'l Ctr Theo Phys, ITAtY Niss Clelia de Balo, Assoc FuRATut-DNEA, IIALY Biblioteca, del ONR EIRAIOM, ITRLY DT. H. Yamato, Toshiha Res \& Dev, JAFts Direc Dept Lg. Tbkamak Deri. MFPr, IRPAN Prof. Notisyiki Inave, University of Tokyo, JApaN zesearch Info Center, Nagoja thiversity. JAPAN Pme. KYoji Nighikam, thiv of firoshima, JAFAN Prof. Sigeru Mori, JABRI, TAPAN i.jbrary, Kyoto University, JAPAN Prof. Iehdro Kanakand, Niton Univ, IAPAN Prof. Satoghi Iton, Kynuht thiversity, JAPAY or. 2. I. Troi, Adv, Inst Sci \& Tech, KOREA Tech Info Divigion, KAEP, KOREA zibliothesk, Fum-Ingt Voor plagma, NEThERLANDS
Pcof. B.S. Liley, Univesity of Haitrato, Noy assand Prof. J.A.C. Cabral, Inst Supetiar Tecn, PCRIURAL DC. Octavian petris, ALI CIZA thiversity, ROMANIA Prof. M.A. Hallberg, University of tatal, SO AFrICA Dc. Johan de villiers, Plagma thysics, Mrcor, so AfRICA Fusion Div. Library, JeN, SPAIN

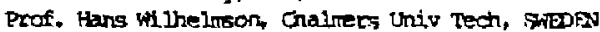
Dr. Lennare Stenflo, University of UMBA, Shipen Library, Royal Inst Tech, SwDDEN Centre is Recherchegen, Eoole polytech Fed, SWITZERMND DT. V.T. Tolok, Marko, Fhys Iech Ins, ISGR DT. D.D. Ayutov, Siberian Acad Sci, USSR DI. G.A. Eliseev, Kurchatov Institute, ISSR Dr. Y.A. Glukhikh, Inst Electro-Physical, USSR Ingtitute Gen. Physica, CSSR

Prof. T.J.M. Boyt, unit College N Nales, WALFs Dr. Ko Schindler, Rahe Universitat, W. GEFfary Muclear Res Bstab, Julich Ltj, Iibxarian, ax-pland Instial, w. GEPMANY Bibliothek, Inst Plasmaforschung, w. GBPABNY Prof. R.K. Janev, Inst Fhys, VusosiduIA 